\title{
LOOP GROUPS AND TWISTED $K$-THEORY I
}

\author{
DANIEL S. FREED, MICHAEL J. HOPKINS, AND CONSTANTIN TELEMAN
}

\section{CONTENTs}

Introduction

1. Twisted $K$-theory by example

2. Twistings of $K$-theory

2.1. Graded T-bundles

2.2. Graded central extensions

2.3. Twistings

2.4. Examples of twistings

3. Twisted K-groups

3.1. Axioms

3.2. Twisted Hilbert spaces

3.3. Universal Twisted Hilbert Bundles

3.4. Definition of twisted $K$-groups

3.5. Verification of the axioms

3.6. The Thom isomorphism, pushforward, and the Pontryagin product

3.7. The fundamental spectral sequence

4. Computation of $K_{G}^{\tau}(G)$

4.1. Notation and assumptions

4.2. The main computation

4.3. The action of $W_{a f f}^{e}$ on $\mathfrak{t}$

Appendix A. Groupoids

A.1. Definition and First Properties

A.2. Further Properties of Groupoids

A.3. Fiber bundles over groupoids and descent

A.4. Hilbert bundles

A.5. Fredholm operators and $K$-theory

References

\section{INTRODUCTION}

Equivariant $K$-theory focuses a remarkable range of perspectives on the study of compact Lie groups. One finds tools from topology, analysis, and representation theory brought together in describing the equivariant $K$-groups of spaces and the maps between them. In the process all three points of view are illuminated. Our aim in this series of papers [19, 20] is to begin the development of similar relationships when a compact Lie group $G$ is replaced by $L G$, the infinite dimensional group of smooth maps from the circle to $G$. 
There are several features special to the representation theory of loop groups. First of all, we will focus only on the representations of $L G$ which have "positive energy." This means that the representation space $V$ admits an action of the rotation group of the circle which is (projectively) compatible with the action of $L G$, and for which there are no vectors $v$ on which rotation by $\theta$ acts by multiplication by $e^{i n \theta}$ with $n<0$. It turns out that most positive energy representations are projective, and so $V$ must be regarded as a representation of a central extension $L G^{\tau}$ of $L G$ by $U(1)$. The topological class of this central extension is known as the level. One thing a topological companion to the representation theory of loop groups must take into account is the level.

Next there is the fusion product. Write $\mathrm{R}^{\tau}(L G)$ for the group completion of the monoid of positive energy representations of $L G$ at level $\tau$. In [34, Erik Verlinde introduced a multiplication on $\mathrm{R}^{\tau}(L G) \otimes \mathbb{C}$ making it into a commutative ring (in fact a Frobenius algebra). This multiplication is called fusion, and $\mathrm{R}^{\tau}(L G) \otimes \mathbb{C}$, equipped with the fusion product is known as the Verlinde algebra. The fusion product also makes $\mathrm{R}^{\tau}(L G)$ into a ring which we will call the Verlinde ring. A good topological description of $\mathrm{R}^{\tau}(L G)$ should account for the fusion product in a natural way.

The positive energy representations of loop groups turn out to be completely reducible, and somewhat surprisingly, there are only finitely many irreducible positive energy representations at a fixed level. Moreover, an irreducible positive energy representation is determined by its lowest non-trivial energy eigenspace, $V\left(n_{0}\right)$, which is an irreducible (projective) representation of $G$. Thus the positive energy representations of $L G$ correspond to a subset of the representations of the compact group $G$. This suggests that $G$-equivariant $K$-theory might somehow play a role in describing the representations of $L G$. In fact this is the case. Here is our main theorem.

Theorem 1. Let $G$ be a connected compact Lie group and $\tau$ a level for the loop group. The Grothendieck group $\mathrm{R}^{\tau}(L G)$ at level $\tau$ is isomorphic to a twisted form $K_{G}^{\zeta(\tau)}(G)$, of the equivariant $K$-theory of $G$ acting on itself by conjugation. Under this isomorphism the fusion product, when it is defined, corresponds to the Pontryagin product. The twisting $\zeta(\tau)$ is given in terms of the level

$$
\zeta(\tau)=\mathfrak{g}+\check{h}+\tau,
$$

where $\check{h}$ is the "dual Coxeter" twisting.

Several aspects of this theorem require clarification. The main new element is the "twisted form" of $K$-theory. Twisted $K$-theory was introduced by Donovan and Karoubi [14] in connection with the Thom isomorphism, and generalized and further developed by Rosenberg [29]. Interest in twisted $K$-theory was rekindled by its appearance in the late 1990's [26, 35] in string theory. Our results came about in the wake of this revival when we realized that the work of the first author [17] on Chern-Simons theory for finite groups could be interpreted in terms twisted $K$-theory.

The twisted forms of $G$-equivariant $K$-theory are classified by the nerve of the category of invertible modules over the equivariant $K$-theory spectrum $K_{G}$. What comes up in geometry though, is only a small subspace, and throughout this paper the term "twisting" will refer twistings in this restricted, more geometric class. 
These geometric twistings of $K_{G}$-theory on a $G$-space $X$ are classified up to isomorphism by the set

$$
H_{G}^{0}(X ; \mathbb{Z} / 2) \times H_{G}^{1}(X ; \mathbb{Z} / 2) \times H_{G}^{3}(X ; \mathbb{Z}) .
$$

The component in $H^{0}$ corresponds to the "degree" of a $K$-class, and the fact that the coefficients are the integers modulo 2 is a reflection of Bott periodicity. In this sense "twistings" refine the notion of "degree," though when considering twistings it is important to remember more than just the isomorphism class.

The tensor product of $K_{G}$-modules makes these spaces of twistings into infinite loop spaces and provides a commutative group structure on the sets of isomorphism classes. The group structure on (2) is the product of $H_{G}^{0}(X ; \mathbb{Z} / 2)$ with with the extension of $H_{G}^{1}(X ; \mathbb{Z} / 2)$ by $H_{G}^{3}(X ; \mathbb{Z})$ with cocycle $\beta(x \cup y)$, where $\beta$ is the Bockstein homomorphism.

A twisting is a form of equivariant $K$-theory on a space. A level for the loop group, on the other hand, corresponds to a central extension of $L G$. One way of relating these two structures is via the classification (2). A central extension of $L G$ has a topological invariant $H_{G}^{3}(G)$, and so give rise to a twisting, up to isomorphism. When the group $G$ is simple and simply connected this invariant determines the central extension up to isomorphism, the group $H_{G}^{1}(G)$ vanishes, and there is a canonical isomorphism $H_{G}^{3}(G) \approx \mathbb{Z}$. In this case an integer can be used to specify both a level and a twisting. There is a more refined version of this correspondence directly relating twistings to central extension, and the approach to twistings we take in this paper is designed to make this relationship as transparent as possible.

There is a map from vector bundles to twistings which associates to a vector bundle $V$ over $X$ the family of $K$-modules $K^{\bar{V}_{x}}$, where $\bar{V}_{x}$ is the one point compactification of the fiber of $V$ over $x \in X$, and for a space $S, K^{S}$ is the $K$-module with $\pi_{0} K^{S}=K^{0}(S)$. We denote the twisting associated to $V$ by $\tau_{V}$, though when no confusion is likely to arise we will just use the symbol $V$. The invariants of $\tau_{V}$ in (2) are $\operatorname{dim} V, w_{1}(V)$ and $\beta w_{2}(V)$. These twistings are described by Donovan and Karoubi in 14 from the point of view of Clifford algebras.

There is also a homomorphism from $K O_{G}^{-1}(X)$ to the group of twistings of equivariant $K$-theory on $X$. In topological terms it corresponds to the map from the stable orthogonal group $O$ to its third Postnikov section $O\langle 0, \ldots, 3\rangle$. It sends an element of $K O^{-1}$ to the twisting whose components are $\left(\sigma w_{1}, \sigma w_{2}, x\right)$, where $\sigma: H^{*}(B O) \rightarrow H^{*-1}(O)$ is the cohomology suspension, and $x \in H^{3}(O ; \mathbb{Z})$ is the unique element, twice which is the cohomology suspension of $p_{1}$. In terms of operator algebras this homomorphism sends a skew-adjoint Fredholm operator to its graded Pfaffian gerbe. We will call this map pfaff.

We now describe two natural twistings on $G$ which are equivariant for the adjoint action. The first comes from the adjoint representation of $G$ regarded as an equivariant vector bundle over a point, and pulled back to $G$. We'll write this twisting as $\mathfrak{g}$. For the other, first note that the equivariant cohomology group $H_{G}^{*}(G)$ is just $H^{*}(L B G)$. The vector bundle associated to the adjoint representation gives a class ad $\in K O^{0}(B G)$ which we can transgress to $K O^{-1}(L B G)$, and then map to twistings by the map pfaff. We'll call this twisting $\breve{h}$. When $G$ is simple and simply connected, the integer corresponding to $\breve{h}$ is the dual Coxeter number, and $\mathfrak{g}$ is just a degree shift. With these definitions, the formula

$$
\zeta(\tau)=\mathfrak{g}+\check{h}+\tau
$$


in the statement of Theorem 1 should be clear. The twistings $\mathfrak{g}$ and $\check{h}$ are those just described, and $\tau$ is the twisting corresponding to the level.

Theorem 1 provides a topological description of the group $\mathrm{R}^{\tau}(L G)$ and its fusion product when it exists. But it also gives more. The twisted $K$-group $K_{G}^{\zeta(\tau)}(G)$ is defined for any compact Lie group $G$, and it makes sense for any level $\tau$. This points the way to a formulation of an analogue of the group $\mathrm{R}^{\tau}(L G)$ for any compact Lie group $G$ (even one which is finite). In Parts II and III we take up these generalizations and show that the assertions of Theorem 1 remain true.

One thing that emerges from our topological considerations is the need to consider $\mathbb{Z} / 2$-graded central extensions of loop groups. Such extensions are necessary when working with a group like $S O(3)$ whose adjoint representation is not Spin. Another interesting case is that of $O(2)$. When the adjoint representation is not orientable the dual Coxeter twist makes a non-trivial contribution to $H_{G}^{0}(G ; \mathbb{Z} / 2)$. One sees an extra change in the degree in which the interesting $K$-group occurs. In the case of $O(2)$ these degree shifts are different on the two connected components, again emphasizing the point that twistings should be regarded as a generalization of degree. The "Verlinde ring" in this case is comprised of an even $K$-group on one component and an odd $K$-group on another. Such inhomogeneous compositions are not typically considered when discussing ordinary $K$-groups.

The fusion product on $\mathrm{R}^{\tau}(L G)$ has been defined for simple and simply connected $G$, and in a few further special cases. The Pontryagin product on $K_{G}^{\zeta(\tau)}(G)$ is defined exactly when $\tau$ is primitive in the sense that its pullback along the multiplication map of $G$ is isomorphic to the sum of its pullbacks along the two projections. This explains, for example, why a fusion product on $\mathrm{R}^{\tau}(L S O(n))$ exists only at half of the levels. Using the Pontryagin product we are able to define a fusion product on $\mathrm{R}^{\tau}(L G)$ for any $G$ at any primitive level $\tau$. We do not, however, give a construction of this product in terms of representation theory.

When the fusion product is defined on $\mathrm{R}^{\tau}(L G)$ it is part of a much more elaborate structure. For one thing, there is a trace map $\mathrm{R}^{\tau}(L G) \rightarrow \mathbb{Z}$ making $\mathrm{R}^{\tau}(L G)$ into a Frobenius algebra. Using twisted $K$-theory we construct this trace map for general compact Lie groups at primitive levels $\tau$ which are non-degenerate in the sense that the image of $\tau$ in

$$
H_{T}^{3}(T ; \mathbb{R}) \approx H^{1}(T ; \mathbb{R}) \otimes H^{1}(T ; \mathbb{R})
$$

is a non-degenerate bilinear form. Again, in the cases when the fusion product has been defined, there are operations on $\mathrm{R}^{\tau}(L G)$ coming from the moduli spaces of Riemann surfaces with boundary, making $\mathrm{R}^{\tau}(L G)$ part of is often called a topological conformal field theory. Using topological methods, we are able to construct a topological conformal field theory for any compact Lie group $G$, at levels $\tau$ which are transgressed from (generalized) cohomology classes on $B G$ and which are nondegenerate. Some of this work appears in [21, 18.

Another impact of Theorem 1 is that it brings the computational techniques of algebraic topology to bear on the representations of loop groups. One very interesting approach, for connected $G$, is to use the Rothenberg-Steenrod spectral sequence relating the equivariant $K$-theory of $\Omega G$ to that of $G$. In this case one gets a spectral sequence

$$
\operatorname{Tor}^{K_{*}^{G}(\Omega G)}(R(G), R(G)) \Longrightarrow K_{\tau+*}^{G}(G),
$$


relating the untwisted equivariant $K$-homology of $\Omega G$, and the representation ring of $G$ to the Verlinde algebra. The ring $K_{*}^{G}(\Omega G)$ can be computed using the techniques of Bott [5] and Bott-Samelson [6] and has also been described by Bezrukavnikov, Finkelberg and Mirković [4. The $K$-groups in the $E^{2}$-term are untwisted. The twisting appears in the way that the representation $\operatorname{ring} R(G)$ is made into an algebra over $K^{G}(\Omega G)$. The equivariant geometry of $\Omega G$ has been extensively studied in connection with the representation theory of $L G$, and the spectral sequence (3) seems to express yet another relationship. We do not know of a representation theoretic construction of (3). An analogue of the spectral sequence (3) has been used by Chris Douglas [15, to compute the (non-equivariant) twisted $K$-groups $K^{\tau}(G)$ for all simple, simply connected $G$.

Using the Lefschetz fixed point formula one can easily conclude for connected $G$ that

$$
\Delta^{-1} K_{*}^{G}(\Omega G)=\Delta^{-1} \mathbb{Z}[\Lambda \times \Pi],
$$

where $\Delta$ is the square of the Weyl denominator, $\Pi=\pi_{1} T$ is the co-weight lattice, and $\Lambda$ is the weight lattice. When the level $\tau$ is non-degenerate there are no higher Tor groups, and the spectral sequence degenerates to an isomorphism

$$
\Delta^{-1} K_{\tau+*}^{G}(G) \approx \Delta^{-1} R(G) / I^{\tau}
$$

where $I^{\tau}$ is the ideal of representations whose characters vanish on certain conjugacy classes. The main computation of this paper asserts that such an isomorphism holds without inverting $\Delta$ when $G$ is connected, and $\pi_{1} G$ is torsion free. The distinguished conjugacy classes are known as Verlinde conjugacy classes, and the ideal $I^{\tau}$ as the Verlinde ideal. In [21] the $\operatorname{ring} K_{G}^{\zeta(\tau)}(G) \otimes \mathbb{C}$ is computed using a fixed point formula, and shown to be isomorphic to the Verlinde algebra.

The plan of this series of papers is as follows. In Part I we define twisted $K$-groups, and compute the groups $K_{G}^{\zeta}(G)$ for connected $G$ with torsion free fundamental group, at non-degenerate levels $\zeta$. Our main result is Theorem 4.27 In Part II we introduce a certain family of Dirac operators and our generalization of $\mathrm{R}^{\tau}(L G)$ to arbitrary compact Lie groups. We construct a map from $\mathrm{R}^{\tau}(L G)$ to $K_{G}^{\zeta(\tau)}(G)$ and show that it is an isomorphism when $G$ is connected with torsion free fundamental group. In Part III we show that our map is an isomorphism for general compact Lie groups $G$, and develop some applications.

The bulk of this paper is concerned with setting up twisted equivariant $K$-theory. There are two things that make this a little complicated. For one, when working with twistings it is important to remember the morphisms between them, and not just the isomorphism classes. The twistings on a space form a category and spelling out the behavior of this category as the space varies gets a little elaborate. The other thing has to do with the kind of $G$-spaces we use. We need to define twistings on $G$-equivariant $K$-theory in such a way as to make clear what happens as the group $G$ changes, and for the constructions in Part II we need to make the relationship between twistings and (graded) central extensions as transparent as possible. We work in this paper with groupoids and define twisted equivariant $K$-theory for groupoids. Weakly equivalent groupoids (see Appendix A) have equivalent categories of twistings and isomorphic twisted $K$-groups. A group $G$ acting on a space $X$ forms a special kind of groupoid $X / / G$ called a "global quotient groupoid." A central extension of $G$ by $U(1)$ defines a twisting of $K$-theory for $X / / G$. If $G$ is a compact connected Lie group acting on itself by conjugation, and $P G$ denotes the 
space of paths in $G$ starting at the identity, acted upon by $L G$ by conjugation, then

$$
P G / / L G \rightarrow G / / G
$$

is a local equivalence, and a (graded) central extension of $L G$ defines a twisting of $P G / / L G$ and hence of $G / / G$. In general we will define a twisting of a groupoid $X$ to consist of a local equivalence $P \rightarrow X$ and a graded central extension $\tilde{P}$ of $P$. While most of the results we prove reduce, ultimately, to ordinary results about compact Lie groups acting on spaces, not all do. In Part III it becomes necessary to work with groupoids which are not equivalent to a compact Lie group acting on a space.

Nitu Kitchloo 24 has pointed out that the space $P G$ is the universal $L G$ space for proper actions. Using this he has described a generalization of our computation to other Kac-Moody groups.

At the time we began this work, the paper of Atiyah and Segal [3] was in preparation, and we benefited a great deal from early drafts. Since that time several other approaches to twisted $K$-theory have appeared. In addition to 3 we refer the reader to [10, 33. We have chosen to use "graded central extensions" because of the close connection with loop groups and the constructions we wish to make in Part II. Of course our results can be presented from any of the points of view mentioned above, and the choice of which is a matter of personal preference.

We have attempted to organize this paper so that the issues of implementation are independent of the issues of computation. Section 1 is a kind of field guide to twisted $K$-theory. We describe a series of examples intended to give the reader a working knowledge of twisted $K$-theory sufficient to follow the main computation in 44. Section 2 contains our formal discussion of twistings of $K$-theory for groupoids, and our definition of twisted $K$-groups appears in $₫ 3$. We have attempted to axiomatize the theory of twisted $K$-groups in order to facilitate comparison with other models. Our main computation appears in 4

This paper has been a long time in preparation and we have benefited from discussion with many people. The authors would like to thank Sir Michael Atiyah and Graeme Segal for making available early drafts of [3] and [2. As will be evident to the reader, our approach to twisted $K$-theory relies heavily on their ideas. We would like to thank Is Singer for many useful conversations. We would also like to thank Ulrich Bunke and Thomas Schick for their careful study and comments on this work. The report of their seminar appears in [12].

We assume throughout this paper that all spaces are locally contractible, paracompact and completely regular. These assumptions implies the existence of partitions of unity [13] and locally contractible slices through actions of compact Lie groups [27, 28].

\section{Twisted $K$-Theory By EXAMPle}

The $K$-theory of a space is assembled from data which is local. To give a vector bundle $V$ on $X$ is equivalent to giving vector bundles $V_{i}$ on the open sets $U_{i}$ of a covering, and isomorphisms

$$
\lambda_{i j}: V_{i} \rightarrow V_{j}
$$

on $U_{i} \cap U_{j}$ satisfying a compatibility (cocycle) condition on the triple intersections. In terms of $K$-theory this is expressed by the Mayer-Vietoris (spectral) sequence relating $K(X)$ and the $K$-groups of the intersections of the $U_{i}$. In forming twisted 
$K$-theory we modify this descent or gluing datum, by introducing a line bundle $L_{i j}$ on $U_{i} \cap U_{j}$, and asking for an isomorphism

$$
\lambda_{i j}: L_{i j} \otimes V_{i} \rightarrow V_{j}
$$

satisfying a certain cocycle condition. In terms of $K$-theory, this modifies the restriction maps in the Mayer-Vietoris sequence.

In order to formulate the cocycle condition, the $L_{i j}$ must come equipped with an isomorphism

$$
L_{j k} \otimes L_{i j} \rightarrow L_{i k}
$$

on the triple intersections, satisfying an evident compatibility relation on the quadruple intersections. In other words, the $\left\{L_{i j}\right\}$ must form a 1-cocycle with values in the groupoid of line bundles. Cocycles differing by a 1-cochain give isomorphic twisted $K$-groups, so, up to isomorphism, we can associate a twisted notion of $K(X)$ to an element

$$
\tau \in H^{1}(X ;\{\text { Line Bundles }\}) .
$$

On good spaces there are isomorphisms

$$
H^{1}(X ;\{\text { Line Bundles }\}) \approx H^{2}(X ; U(1)) \approx H^{3}(X ; \mathbb{Z}),
$$

and correspondingly, twisted notions of $K$-theory associated to an integer valued 3 -cocycle. In this paper we find we need to allow the $L_{i j}$ to be \pm line bundles, so in fact we consider twisted notion of $K(X)$ classified by element: 1$]$

$$
\tau \in H^{1}(X ;\{ \pm \text { Line Bundles }\}) \approx H^{3}(X ; \mathbb{Z}) \times H^{1}(X ; \mathbb{Z} / 2) .
$$

We will write $K^{\tau+n}$ for the version of $K^{n}$, twisted by $\tau$.

In practice, to compute twisted $K(X)$ one represents the twisting $\tau$ as a Cech 1 -cocycle on an explicit covering of $X$. The twisted $K$-group is then assembled from the Mayer-Vietoris sequence of this covering, involving the same (untwisted) $K$-groups one would encounter in computing $K(X)$. The presence of the 1-cocycle is manifest in the restriction maps between the $K$-groups of the open sets. They are modified on the two-fold intersections by tensoring with the $( \pm)$ line bundle given by the 1-cocycle. This "operational definition" suffices to make most computations. See $\$ 3$ for a more careful discussion. Here are a few examples.

Example 1.4. Suppose that $X=S^{3}$, and that the isomorphism class of $\tau$ is $n \in$ $H^{3}(X ; \mathbb{Z}) \approx \mathbb{Z}$. Let $U_{+}=X \backslash(0,0,0,-1)$ and $U_{-}=X \backslash(0,0,0,1)$. Then $U_{+} \cap U_{-} \sim$ $S^{2}$, and $\tau$ is represented by the 1 -cocycle whose value on $U_{+} \cap U_{-}$is $L^{n}$, with $L$ the tautological line bundle. The Mayer-Vietoris sequence for $K^{\tau}(X)$ takes the form

$$
\begin{aligned}
\cdots \rightarrow K^{\tau+0}(X) \rightarrow K^{\tau+0}\left(U_{+}\right) \oplus K^{\tau+0}\left(U_{-}\right) \rightarrow K^{\tau+0}\left(U_{+} \cap U_{-}\right) \\
\quad \rightarrow K^{\tau+1}(X) \rightarrow K^{\tau+1}\left(U_{+}\right) \oplus K^{\tau+1}\left(U_{-}\right) \rightarrow K^{\tau+1}\left(U_{+} \cap U_{-}\right) \rightarrow \cdots .
\end{aligned}
$$

\footnotetext{
${ }^{1}$ The set of isomorphism classes of twistings has a group structure induced from the tensor product of graded line bundles. While there is, as indicated, a set-theoretic factorization of the isomorphism classes of twistings, the group structure is not, in general, the product.
} 
Since the restriction of $\tau$ to $U_{ \pm}$is isomorphic to zero, we have

$$
\begin{aligned}
K^{\tau+0}\left(U_{ \pm}\right) & \approx K^{0}\left(U_{ \pm}\right) \approx \mathbb{Z} \\
K^{\tau+1}\left(U_{ \pm}\right) & \approx K^{1}\left(U_{ \pm}\right) \approx 0 \\
K^{\tau+1}\left(U_{+} \cap U_{-}\right) & \approx K^{1}\left(S^{2}\right) \approx 0,
\end{aligned}
$$

and

$$
K^{\tau+0}\left(U_{+} \cap U_{-}\right) \approx K^{0}\left(S^{2}\right) \approx \mathbb{Z} \oplus \mathbb{Z}
$$

with basis the trivial bundle 1 , and the tautological line bundle $L$. The MayerVietoris sequence reduces to the exact sequence

$$
0 \rightarrow K^{\tau+0}(X) \rightarrow \mathbb{Z} \oplus \mathbb{Z} \rightarrow \mathbb{Z} \oplus \mathbb{Z} \rightarrow K^{\tau+1}(X) \rightarrow 0 .
$$

In ordinary (untwisted) $K$-theory, the middle map is

$$
\left(\begin{array}{cc}
1 & -1 \\
0 & 0
\end{array}\right): \mathbb{Z} \oplus \mathbb{Z} \rightarrow \mathbb{Z} \oplus \mathbb{Z}
$$

In twisted $K$-theory, with suitable conventions, the middle map becomes

$$
\left(\begin{array}{cc}
1 & n-1 \\
0 & -n
\end{array}\right): \mathbb{Z} \oplus \mathbb{Z} \rightarrow \mathbb{Z} \oplus \mathbb{Z}
$$

and so

$$
K^{\tau+n}\left(S^{3}\right)= \begin{cases}0 & n=0 \\ \mathbb{Z} / n & n=1 .\end{cases}
$$

In the language of twistings, the map (1.5) is accounted for as follows. To identify the twisted $K$-groups with ordinary twisted $K$-groups we have to choose isomorphisms

$$
t_{ \pm}:\left.\tau\right|_{U_{ \pm}} \rightarrow 0
$$

If we use the $t_{+}$to trivialize $\tau$ on $U_{+} \cap U_{-}$, then the following diagram commutes

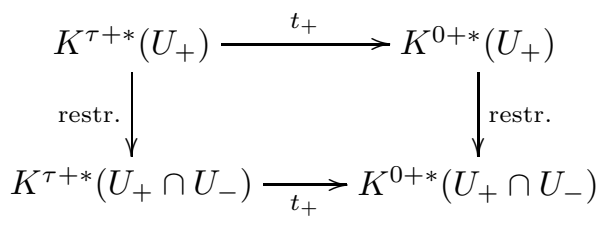

and we can identify the restriction map in twisted $K$-theory from $U_{+}$to $U_{+} \cap U_{-}$ with the restriction map in untwisted $K$-theory. On $U_{+} \cap U_{-}$we have $t_{-}=\left(t_{-} t_{+}^{-1}\right) \circ$ $t_{+}$so the restriction map in twisted $K$-theory is identified with the restriction map in untwisted $K$-theory, followed by the map $\left(t_{-} t_{+}^{-1}\right)$. By definition of $\tau$, this map is given by multiplication by $L^{n}$. This accounts for the second column of (1.5).

Example 1.6. Now consider the twisted $K$-theory of $U(1)$ acting trivially on itself. In this case the twistings are classified by

$$
H_{U(1)}^{3}(U(1) ; \mathbb{Z}) \times H_{U(1)}^{1}(U(1) ; \mathbb{Z} / 2) \approx \mathbb{Z} \oplus \mathbb{Z} / 2 .
$$

We consider twisted $K$-theory, twisted by $\tau=(n, \epsilon)$. Regard $U(1)$ as the unit circle in the complex plane, and set

$$
U_{+}=U(1) \backslash\{-1\} \quad U_{-}=U(1) \backslash\{+1\} .
$$


The twisting $\tau$ restricts to zero on both $U_{+}$and $U_{-}$. Write

$$
K_{U(1)}^{0}=R\left({ }^{1}\right)=\mathbb{Z}\left[L, L^{-1}\right] .
$$

Then the Mayer-Vietoris sequence becomes

$$
0 \rightarrow K_{U(1)}^{\tau+0}(U(1)) \rightarrow \mathbb{Z}\left[L^{ \pm 1}\right] \oplus \mathbb{Z}\left[L^{ \pm 1}\right] \rightarrow \mathbb{Z}\left[L^{ \pm 1}\right] \oplus \mathbb{Z}\left[L^{ \pm 1}\right] \rightarrow K_{U(1)}^{\tau+1}(U(1)) \rightarrow 0 .
$$

The 1-cocycle representing $\tau$ can be taken to be the equivariant vector bundle whose fiber over $-i$ is the trivial representation of $U(1)$ and whose fiber over $+i$ is $(-1)^{\epsilon} L^{n}$. With suitable conventions, the middle map becomes

$$
\left(\begin{array}{cc}
1 & -(-1)^{\epsilon} L^{n} \\
1 & -1
\end{array}\right): \mathbb{Z}\left[L^{ \pm 1}\right]^{2} \rightarrow \mathbb{Z}\left[L^{ \pm 1}\right]^{2}
$$

It follows that

$$
K_{U(1)}^{\tau+k}(U(1))= \begin{cases}0 & k=0 \\ \mathbb{Z}\left[L^{ \pm 1}\right] /\left((-1)^{\epsilon} L^{n}-1\right) & k=1 .\end{cases}
$$

When $\epsilon=0$ this coincides the Grothendieck group of representations of the Heisenberg extension of $\mathbb{Z} \times U(1)$ of level $n$, and in turn with the Grothendieck group of positive energy representations the loop group of $U(1)$ at level $n$.

Example 1.7. Consider the twisted $K$-theory of $S U(2)$ acting on itself by conjugation. The group $H_{S U(2)}^{1}(S U(2) ; \mathbb{Z} / 2)$ vanishes, while

$$
H_{S U(2)}^{3}(S U(2) ; \mathbb{Z})=\mathbb{Z}
$$

so a twistings $\tau$ in this case is given by an integer

$$
n \in H_{S U(2)}^{3}(S U(2) ; \mathbb{Z})=\mathbb{Z} \text {. }
$$

Set

$$
U_{+}=S U(2) \backslash\{-1\} \quad U_{-}=S U(2) \backslash\{+1\} .
$$

The spaces $U_{+}$and $U_{1}$ are equivariantly contractible, while $U_{+} \cap U_{-}$is equivariantly homotopy equivalent to $S^{2}=S U(2) / T$, where $T=U(1)$ is a maximal torus. The restrictions of $\tau$ to $U_{+}$and $U_{-}$are isomorphic to zero. We have

$$
K_{S U(2)}^{0}\left(U_{ \pm}\right) \approx K_{S U(2)}^{0}(\mathrm{pt})=R(S U(2))=\mathbb{Z}\left[L, L^{-1}\right]^{W}
$$

with the Weyl group $W \approx \mathbb{Z} / 2$ acting by exchanging $L$ and $L^{-1}$, and

$$
K_{S U(2)}^{0}\left(U_{+} \cap U_{-}\right) \approx K_{S U(2)}^{0}(S U(2) / T) \approx K_{T}^{0}(\mathrm{pt}) \approx \mathbb{Z}\left[L, L^{-1}\right] .
$$

The ring $R(S U(2))$ has an additive basis consisting of the irreducible representations,

$$
\rho_{k}=L^{k}+L^{k-2}+\cdots+L^{-k}, \quad k \geq 0
$$

which multiply according to the Clebsch-Gordon rule

$$
\rho_{l} \rho_{k}=\rho_{k+l}+\rho_{k+l-2}+\cdots+\rho_{k-l} \quad k \geq l .
$$

As in our other example, the Mayer-Vietoris sequence is short exact

$$
0 \rightarrow K_{S U(2)}^{\tau+0}(S U(2)) \rightarrow R(S U(2)) \oplus R(S U(2)) \rightarrow \mathbb{Z}\left[L^{ \pm 1}\right] \rightarrow K_{S U(2)}^{\tau+1}(S U(2)) \rightarrow 0 .
$$

The 1-cocycle representing the difference between the two trivializations of the restriction of $\tau$ to $U_{+} \cap U_{-}$can be taken to be the element $L^{n} \in K_{S U(2)}(S U(2) / T) \approx$ 
$R(T)$. The sequence is a sequence of $R(S U(2))$-modules. With suitable conventions, the middle map is

$$
\left(1 \quad-L^{n}\right): R(S U(2))^{2} \rightarrow R(T)
$$

To calculate the kernel and cokernel, note that $R(T)$ is a free module of rank 2 over $R(S U(2))$. We give $R(S U(2)) \oplus R(S U(2)))$ the obvious basis, and $R(T)$ the basis $\{1, L\}$. It follows from the identity

$$
L^{n}=L \rho_{n-1}-\rho_{n-2}
$$

that (1.8) is represented by the matrix

$$
\left(\begin{array}{cc}
1 & \rho_{n-2} \\
0 & -\rho_{n-1}
\end{array}\right)
$$

and that

$$
K_{S U(2)}^{\tau+k}(S U(2))= \begin{cases}0 & k=0 \\ R(S U(2)) /\left(\rho_{n-1}\right) & k=1 .\end{cases}
$$

This coincides the Grothendieck group of positive energy representations the loop group of $S U(2)$ at level $(n-2)$.

Examples 1.6 and 1.7 illustrate the relationship between twisted $K$-theory and the representations of loop groups. In both cases the Grothendieck group of positive energy representations of the loop group of a compact Lie group $G$ is described by the twisted equivariant $K$-group of $G$ acting on itself by conjugation. Two minor discrepancies appear in this relationship. On one hand, the interesting $K$-group is in degree $k=1$. As explained in the introduction, the representations of the loop group at level $\tau$ correspond to twisted $K$-theory at the twisting $\zeta(\tau)=\mathfrak{g}+\check{h}+\tau$. The shift in $K$-group to $k=1$ corresponds to the term $\mathfrak{g}$. In both examples the adjoint representation is $\operatorname{Spin}^{c}$ and so contributes only its dimension to $\zeta(\tau)$. This term could be gotten rid of by working with twisted equivariant $K$-homology rather than $K$-cohomology. We have chosen to work with $K$-cohomology in order to make better contact with our geometric constructions in Parts II and III. The other discrepancy is the shift in level in Example 1.7 twisted equivariant $K$-theory at level $n$ corresponds to the representations of the loop group at level $(n-2)$. The shift of 2 here corresponds to the term $\check{h}$ in our formula for $\zeta(\tau)$.

We now give a series of examples describing other ways in which twistings of $K$-theory arise.

Example 1.9. Let $V$ be a vector bundle of dimension $n$ over a space $X$, and write $X^{V}$ for the Thom complex of $V$. Then $\tilde{K}^{n+k}\left(X^{V}\right)$ is a twisted form of $K^{k}(X)$. To identify the twisting, choose local Spin ${ }^{c}$ structures $\mu_{i}$ on the restrictions $V_{i}=\left.V\right|_{U_{i}}$ of $V$ to the sets in an open cover of $X$. The $K$-theory Thom classes associated to the $\mu_{i}$ allow one to identify

$$
\tilde{K}^{n+k}\left(U_{i}^{V_{i}}\right) \approx K^{k}\left(U_{i}\right)
$$

The difference between the two identifications on $U_{i} \cap U_{j}$ is given by multiplication by the graded line bundle representing the difference between $\mu_{i}$ and $\mu_{j}$. Figuratively, the cocycle representing the twisting is $\mu_{j} \mu_{i}^{-1}$ and the cohomology class is $\left(w_{1}(V), W_{3}(V)\right) \in H^{1}(X ; \mathbb{Z} / 2) \times H^{3}(X ; \mathbb{Z})$, where $W_{3}=\beta w_{2}$. This is one of the original examples of twisted $K$-theory, described by Donovan and Karoubi 14 from the point of view of Clifford algebras. We will review their description in 33.6 
Example 1.10. Let $G$ be a compact Lie group. The central extensions

$$
\mathbb{T} \rightarrow \tilde{G} \stackrel{\tau}{\rightarrow} G
$$

of $G$ by $\mathbb{T}=U(1)$ are classified by $H_{G}^{3}(\{\mathrm{pt}\} ; \mathbb{Z})=H^{3}(B G ; \mathbb{Z})$. The Grothendieck group $\mathrm{R}^{\tau}(G)$, of representations of $\tilde{G}$ on which $\mathbb{T}$ acts according to its defining representation, can be thought of as a twisted form of $\mathrm{R}(G)$. In this case, our definition of equivariant twisted $K$-theory gives

$$
K_{G}^{\tau+k}(\mathrm{pt})= \begin{cases}\mathrm{R}^{\tau}(G) & k=0 \\ 0 & k=1\end{cases}
$$

More generally, if $S$ is a $G$-space, and $\tau \in H_{G}^{3}(S)$ is pulled back from $H_{G}^{3}$ (pt), then $K_{G}^{\tau+k}(S)$ is the summand of $K_{\tilde{G}}^{k}(S)$ corresponding to $\tilde{G}$-equivariant vector bundles on which $\mathbb{T}$ acts according to its defining character.

Example 1.11. Now suppose that

$$
H \rightarrow G \rightarrow Q
$$

is an extension of groups, and $V$ is an irreducible representation of $H$ that is stable, up to isomorphism, under conjugation by elements of $G$. Then Grothendieck group of representations of $G$ whose restriction to $H$ is $V$-isotypical, forms a twisted version of the Grothendieck group of representations of $Q$. When $H$ is central, equal to $\mathbb{T}$, and $V$ is the defining representation, this is the situation of Example 1.10 . We now describe how to reduce to this case.

Fix an $H$-invariant Hermitian metric on $V$, and write $V^{*}=\operatorname{hom}(V, \mathbb{C})$ for the representation dual to $V$. Let $\tilde{G}$ denote the group of pairs

$$
(g, f) \in G \times \operatorname{hom}\left(V^{*}, V^{*}\right)
$$

for which $f$ is unitary, and satisfies

$$
f(h v)=g h g^{-1} f(v) \quad h \in H .
$$

Since $V$ is irreducible, and $(\operatorname{ad} g)^{*} V \approx V$, the same is true of $V^{*}$, and the map

$$
\begin{aligned}
\tilde{G} & \rightarrow G \\
(g, f) & \mapsto g
\end{aligned}
$$

is surjective, with kernel $\mathbb{T}$. The inclusion

$$
\begin{aligned}
H & \subset \tilde{G} \\
h & \mapsto(h, \text { action of } h)
\end{aligned}
$$

is normal, and lifts the inclusion of $H$ into $G$. We define

$$
\tilde{Q}=\tilde{G} / H \text {. }
$$

The group $\tilde{Q}$ is a central extension of $Q$ by $\mathbb{T}$, which we denote

$$
\tilde{Q} \stackrel{\tau}{\rightarrow} Q .
$$

We now describe an equivalence of categories between $V$-isotypical $G$-representations, and $\tau$-projective representation of $Q$ (representations of $\tilde{Q}$ on which $\mathbb{T}$ acts according to its defining character). 
By definition, the representations $V$ and $V^{*}$ of $H$ come equipped with extensions to unitary representation of $\tilde{G}$. Given a $V$-isotypical representation $W$ of $G$, we let $M$ denote the $H$-invariant part of $V^{*} \otimes W$ :

$$
M=\left(V^{*} \otimes W\right)^{H} .
$$

The action of $\tilde{G}$ on $V^{*} \otimes W$ factors through an action of $\tilde{Q}$ on $M$. This defines a functor from $V$-isotypical representations of $G$ to $\tau$-projective representations of $Q$.

Conversely, suppose $M$ is a $\tau$-projective representation of $\tilde{Q}$. Let $\tilde{G}$ act on $M$ through the projection $\tilde{G} \rightarrow \tilde{Q}$, and form

$$
W=V \otimes M
$$

The central $\mathbb{T}$ of $\tilde{G}$ acts trivially on $W$, giving $W$ a $G$-action. This defines a functor from the category of $\tau$-projective representations of $Q$ to the category of $V$-isotypical representations of $G$. One easily checks these two functors to form an equivalence of categories.

Example 1.12. Continuing with the situation of Example1.11 consider an extension

$$
H \rightarrow G \rightarrow Q
$$

and an irreducible representation $V$ of $H$, which this time is not assumed to be stable under conjugation by $G$. Write

$$
G_{0}=\left\{g \in G \mid(\operatorname{ad} g)^{*} V \approx V\right\},
$$

and $Q_{0}=G_{0} / H$. Let $S$ be the set of isomorphism classes of irreducible representations of $H$ of the form $(\operatorname{ad} g)^{*} V$. The conjugation action of $G$ on $S$ factors through $Q$, and we have an identification $S=Q / Q_{0}$. Let's call a representation of $G S$-typical if its restriction to $H$ involves only the irreducible representations in $S$. One easily checks that "induction" and "passage to the $V$-isotypical part of the restriction" give an equivalence of categories

$\{S$-typical representations of $G\} \leftrightarrow\left\{V\right.$-isotypical representations of $\left.G_{0}\right\}$,

and therefore an isomorphism of the Grothendieck group $\mathrm{R}^{S}(G)$ of $S$-typical representations of $G$ with

$$
\mathrm{R}^{\tau}\left(Q_{0}\right) \approx K_{Q_{0}}^{\tau}(\mathrm{pt})
$$

We can formulate this isomorphism a little more cleanly in the language of groupoids. For each $\alpha \in S$, choose an irreducible $H$-representation $V_{\alpha}$ representing $\alpha$. Consider the groupoid $S / / Q$, with set of objects $S$, and in which a morphism $\alpha \rightarrow \beta$ is an element $g \in Q$ for which $(\operatorname{ad} g)^{*} \alpha=\beta$. We define a new groupoid $P$ with objects $S$, and with $P(\alpha, \beta)$ the set of equivalences classes of pairs $(g, \phi) \in G \times \operatorname{hom}\left(V_{\alpha^{*}, V_{\beta}}^{*}\right)$, with $\phi$ unitary, and satisfying

$$
\phi(h v)=g h g^{-1} \phi(v)
$$

(so that, among other things, $(\operatorname{ad} g)^{*} \alpha=\beta$ ). The equivalence relation is generated by

$$
(g, \phi) \sim(h g, h \phi) \quad h \in H .
$$

There is an evident functor $\tau: P \rightarrow S / / Q$, representing $P$ as a central extension of $S / / Q$ by $\mathbb{T}$. The automorphism group of $V$ in $P$ is the central extension $\tilde{Q}_{0}$ of $Q_{0}$. An easy generalization of the construction of Example 1.11 gives an equivalence of categories

$\{\tau$ - projective representations of $P\} \leftrightarrow\{S-$ typical representations of $G\}$. 
Central extensions of $S / / Q$ are classified by

$$
H^{3}(S / / Q ; \mathbb{Z})=H_{Q}^{3}(S ; \mathbb{Z}) \approx H_{Q_{0}}^{3}(\mathrm{pt} ; \mathbb{Z}),
$$

and so represent twistings of $K$-theory. Our definition of twisted $K$-theory of groupoids will identify the $\tau$-twisted $K$-groups of $(S / / Q)$ with the summand of the $K$-theory of $P$ on which the central $\mathbb{T}$ acts according to its defining representation. We therefore have an isomorphism

$$
\mathrm{R}^{S}(G) \approx K^{\tau+0}(S / / Q)=K_{Q}^{\tau+0}(S) .
$$

Example 1.13. Now let $S$ denote the set of isomorphism classes of all irreducible representations of $H$. Decomposing $S$ into orbit types, and using the construction of Example 1.12 gives a central extension $\tau: P \rightarrow(S / / Q)$, and an isomorphism

$$
\mathrm{R}(G) \approx K^{\tau+0}(S / / Q)=K_{Q}^{\tau+0}(S) .
$$

More generally, if $X$ is a space with a $Q$-action there is an isomorphism

$$
K_{G}^{k}(X) \approx K_{Q}^{\tau+k}(X \times S),
$$

in which $\tau$ is the $Q$-equivariant twisting of $X \times S$ pulled back from the $Q$-equivariant twisting $\tau$ of $S$, given by $P$.

\section{Twistings OF $K$-THEORY}

We now turn to a more careful discussion of twistings of $K$-theory. Our terminology derives from the situation of Example 1.10 in which a central extension of a group gives rise to a twisted notion of equivariant $K$-theory. By working with graded central extensions of groupoids (rather that groups) we are able to include in a single point of view both the twistings that come from 1-cocycles with values in the group of $\mathbb{Z} / 2$-graded line bundles and the twistings that come from central extensions. In order to facilitate this, in the rest of this paper we will use the language of $\mathbb{T}$-bundles and $\mathbb{T}$-torsors instead of "line bundles," where $\mathbb{T}$ is the group $U(1)$. We begin with a formal discussion of $(\mathbb{Z} / 2$-) graded $\mathbb{T}$-bundles.

2.1. Graded $\mathbb{T}$-bundles. Let $X$ be a topological space.

Definition 2.1. A graded $\mathbb{T}$-bundle over $X$ consists of a principal $\mathbb{T}$-bundle $P \rightarrow X$, and a locally constant function $\epsilon: X \rightarrow \mathbb{Z} / 2$.

We will call a graded $\mathbb{T}$-bundle $(P, \epsilon)$ even (resp. odd) if $\epsilon$ is the constant function function 1 (resp. -1 ). The collection of graded $\mathbb{T}$-bundles forms a symmetric monoidal groupoid. A map of graded T-bundles $\left(P_{1}, \epsilon_{1}\right) \rightarrow\left(P_{1}, \epsilon_{2}\right)$ exists only when $\epsilon_{1}=\epsilon_{2}$, in which case it is a map of principal bundles $P_{1} \rightarrow P_{2}$. The tensor structure is given by

$$
\left(P_{1}, \epsilon_{1}\right) \otimes\left(P_{2}, \epsilon_{2}\right)=\left(P_{1} \otimes P_{2}, \epsilon_{1}+\epsilon_{2}\right),
$$

in which $P_{1} \otimes P_{2}$ is the usual "tensor product" of principal $\mathbb{T}$-bundles:

$$
\left(P_{1} \otimes P_{2}\right)_{x}=\left(P_{1}\right)_{x} \times\left(P_{2}\right)_{x} /(v \lambda, w) \sim(v, w \lambda) .
$$

It is easiest to describe the symmetry transformation

$$
T:\left(P_{1}, \epsilon_{1}\right) \otimes\left(P_{2}, \epsilon_{2}\right) \rightarrow\left(P_{2}, \epsilon_{2}\right) \otimes\left(P_{1}, \epsilon_{1}\right)
$$

fiberwise. In the fiber over a point $x \in X$ it is

$$
(v, w) \mapsto\left(w, v \epsilon_{1}(x) \epsilon_{2}(x)\right) .
$$


We will write $\mathcal{B} \mathbb{T}^{ \pm}$for the contravariant functor which associates to a space $X$ the category of graded $\mathbb{T}$-bundles over $X$, and for $\mathcal{B} \mathbb{T}$ the functor "category of $\mathbb{T}$ bundles". We will also write $H^{1}\left(X ; \mathbb{T}^{ \pm}\right)$for the group of isomorphism classes in $\mathcal{B} \mathbb{T}^{ \pm}(X)$, and $H^{0}\left(X ; \mathbb{T}^{ \pm}\right)$for the group of automorphisms of any object. There is an exact sequence

$$
\mathcal{B} \mathbb{T} \rightarrow \mathcal{B} \mathbb{T}^{ \pm} \rightarrow Z / 2
$$

in which the rightmost arrow is "forget everything but the grading." In fact this sequence can be split by associating to a locally constant function $\epsilon: X \rightarrow \mathbb{Z} / 2$ the "trivial" graded T-bundle

$$
\mathbf{1}^{\epsilon}:=(X \times \mathbb{T}, \epsilon) .
$$

This splitting is compatible with the monoidal structure, but not with its symmetry. It gives an equivalence

$$
\mathcal{B} \mathbb{T}^{ \pm} \approx \mathcal{B} \mathbb{T} \times \mathbb{Z} / 2
$$

of monoidal categories (but not of symmetric monoidal categories). It follows that the group $H^{1}\left(X ; \mathbb{T}^{ \pm}\right)$, of isomorphism classes of graded $\mathbb{T}$-bundles over $X$, is isomorphic to $H^{0}(X ; \mathbb{Z} / 2) \times H^{1}(X ; \mathbb{T})$. Since $X$ is assumed to be paracompact, this in turn is isomorphic to $H^{0}(X ; \mathbb{Z} / 2) \times H^{2}(X ; \mathbb{Z})$. The automorphism group of any graded $\mathbb{T}$-bundle is the group of continuous maps from $X$ to $\mathbb{T}$.

2.2. Graded central extensions. Building on the notion of graded $\mathbb{T}$-bundles we now turn to graded central extension of groupoids. The reader is referred to Appendix A for our conventions on groupoids, and a recollection of the fundamental notions. Unless otherwise stated all groupoids will be assumed to be local quotient groupoids ( $\mathrm{A} .2 .2$ ), in the sense that they admit a countable open cover by subgroupoids, each of which is weakly equivalent to a compact Lie group acting on a Hausdorff space.

Let $X=\left(X_{0}, X_{1}\right)$ be a groupoid. Write $\mathcal{B} \mathbb{Z} / 2$ for the groupoid associated to the action of $\mathbb{Z} / 2$ on a point.

Definition 2.4. A graded groupoid is a groupoid $X$ equipped with a functor $\epsilon$ : $X \rightarrow \mathcal{B Z} / 2$. The map $\epsilon$ is called the grading.

The collection of gradings on $X$ forms a groupoid, in which a morphism is a natural transformation. Spelled out, a grading of $X$ is a function $\epsilon: X_{1} \rightarrow \mathbb{Z} / 2$ satisfying $\epsilon(g \circ f)=\epsilon(g)+\epsilon(f)$, and a morphism from $\epsilon_{0}$ to $\epsilon_{1}$ is a continuous function $\eta: X_{0} \rightarrow \mathbb{Z} / 2$ satisfying, for each $(f: x \rightarrow y) \in X_{1}$,

$$
\epsilon_{1}(f)=\epsilon_{0}(f)+(\eta(y)-\eta(x)) .
$$

Example 2.5. Suppose that $X=S / / G$, with $S$ a connected topological space. Then a grading of $X$ is just a homomorphism $G \rightarrow \mathbb{Z} / 2$, making $G$ into a graded group.

We denote the groupoid of gradings of $X$

$$
\mathfrak{H o m}(X, \mathcal{B Z} / 2) \text {. }
$$

Definition 2.6. A graded central extension of $X$ is a graded $\mathbb{T}$-bundle $L$ over $X_{1}$, together with an isomorphism of graded $\mathbb{T}$-bundles on $X_{2}$

$$
\lambda_{g, f}: L_{g} \otimes L_{f} \rightarrow L_{g \circ f}
$$


satisfying the cocycle condition, that the diagram

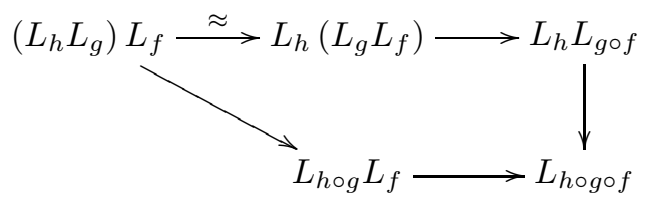

of graded $\mathbb{T}$-bundles on $X_{3}$ commutes.

If $L \rightarrow X_{1}$ is a graded central extension of $X$, then the pair $\tilde{X}=\left(X_{0}, L\right)$ is a graded groupoid over $X$, and the functor $\tilde{X} \rightarrow X$ represents $\tilde{X}$ as a graded central extension of $X$ by $\mathbb{T}$ in the evident sense. Our terminology comes from this point of view. Some constructions are simpler to describe in terms of the graded $\mathbb{T}$-bundles $L$ and others in terms of $\tilde{X} \rightarrow X$.

The collection of graded central extensions of $X$ forms a symmetric monoidal 2-category which we denote $\underline{\mathfrak{E x t}}\left(X, \mathbb{T}^{ \pm}\right)=\underline{\mathfrak{E x t}}_{X}$. The category of morphisms in $\mathbb{E x t}_{X}$ from $L^{1} \rightarrow L^{2}$ is the groupoid of graded $\mathbb{T}$-torsors $(\eta, \epsilon)$ over $X_{0}$, equipped with an isomorphism

$$
\eta_{b} L_{f}^{1} \rightarrow L_{f}^{2} \eta_{a}
$$

making

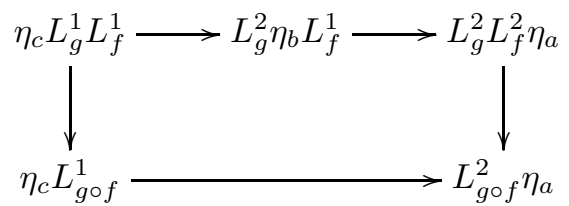

commute. The tensor product $L \otimes L^{\prime}$ is the graded central extension

$$
L \otimes L^{\prime} \rightarrow X_{1}
$$

with structure map

$$
L_{g} \otimes L_{g}^{\prime} \otimes L_{f} \otimes L_{f}^{\prime} \stackrel{1 \otimes \mathrm{T} \otimes 1}{\longrightarrow} L_{g} \otimes L_{f} \otimes L_{g}^{\prime} \otimes L_{f}^{\prime} \stackrel{\lambda_{g, f} \otimes \lambda_{g, f}^{\prime}}{\longrightarrow} L_{g \circ f} \otimes L_{g \circ f}^{\prime} .
$$

The symmetry isomorphism $L \otimes L^{\prime} \rightarrow L^{\prime} \otimes L$ is derived from the symmetry of the tensor product of graded $\mathbb{T}$-bundles.

For the purposes of twisted $K$-groups it suffices to work with the 1-category quotient of $\underline{\mathfrak{E x t}}_{X}$.

Definition 2.7. The category $\mathfrak{E x t}\left(X ; \mathbb{T}^{ \pm}\right)=\mathfrak{E x t}_{X}$ is the category with objects the graded central extensions of $X$, and with morphisms from $L$ to $L^{\prime}$ the set of isomorphism classes in $\underline{\mathfrak{E x t}}_{X}\left(L, L^{\prime}\right)$.

The symmetric monoidal structure on $\underline{\mathfrak{r x t}}_{X}$ makes $\mathfrak{E} \mathfrak{x t}{ }_{X}$ into a symmetric monoidal groupoid in the evident way.

Remark 2.8. A 1-automorphisms of $L$ consists of a graded $\mathbb{T}$ torsor $\eta$ over $X_{0}$, together with an isomorphism $\eta_{a} \rightarrow \eta_{b}$ over $X_{1}$, satisfying the cocycle condition. In this way the category of automorphisms of any twisting can be identified with the groupoid of graded line bundles over $X$. A graded line bundle on $X$ defines an element of $K^{0}(X)$ (even line bundles go to line bundles, and odd line bundles go to their negatives). The fundamental property relating twistings and twisted $K$-theory is that the automorphism $\eta$ acts on twisted $K$-theory as multiplication by the corresponding element of $K^{0}(X)$ (Proposition 3.3). 
Remark 2.9. (1) The formation of $\mathfrak{E x t}_{X}$ is functorial in $X$, in the sense of 2 categories. If $F: Y \rightarrow X$ is a map of groupoids, and $L \rightarrow X_{1}$ is a graded central extension of $X$, then $F^{*} L \rightarrow Y_{1}$ gives a graded central extension, $F^{*} L$ of $Y$. If $\eta \rightarrow X_{0}$ is a morphism from $L^{1}$ to $L^{2}$, then $F^{*} \eta$ defines a morphism from $F^{*} L^{1}$ to $F^{*} L^{2}$.

(2) If $T: Y_{0} \rightarrow X_{1}$ is a natural transformation from $F$ to $G$, then the graded line bundle $T^{*} L$ determines a morphism from $F^{*} L$ to $G^{*} L$. This is functorial in the sense that given $\eta: L^{1} \rightarrow L^{2}$, there is a 2-morphism relating the two ways of going around

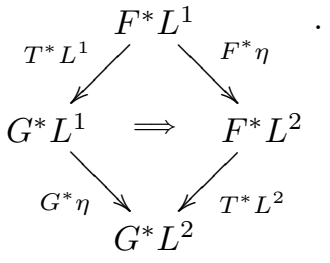

The 2-morphism is the isomorphism

$$
\left(G^{*} \eta\right) \circ\left(T^{*} L^{1}\right) \rightarrow\left(T^{*} L^{2}\right) \circ\left(F^{*} \eta\right)
$$

gotten by pulling back map $\eta: L^{1} \rightarrow L^{2}$ along $T$. It is given pointwise over $y \in Y$, $T y: F y \rightarrow G y$ by

$$
\left(\eta_{G y}\right)\left(L_{T y}^{1}\right) \rightarrow\left(L_{T y}^{2}\right)\left(\eta_{F y}\right) .
$$

(3) It follows that the formation of $\mathfrak{E x t}_{X}$ is functorial in $X$, making $\mathfrak{E x t}_{X}$ a (weak) presheaf of groupoids.

Example 2.10. Suppose that $G$ is a group, and $X=\mathrm{pt} / / G$. Then a graded central extension of $X$ is just a graded central extension of $G$ by $\mathbb{T}$.

Forgetting the $\mathbb{T}$-bundle gives a functor from $\underline{\mathfrak{E x t}}_{X}$ to the groupoid of gradings of $X$, and the decomposition (2.3) gives a 2-category equivalence

$$
\underline{\mathfrak{E} \mathfrak{x t}}\left(X, \mathbb{T}^{ \pm}\right) \approx \underline{\mathfrak{E x t}}(X, \mathbb{T}) \times \mathfrak{H o m}(X, \mathcal{B Z} / 2)
$$

which is not, in general, compatible with the monoidal structure. Here $\mathfrak{E} \mathfrak{x}(X, \mathbb{T})$ is the 2-category of evenly graded (ie, ordinary) central extensions of $X$ by $\mathbb{T}$.

2.2.1. Classification of graded central extensions. We now turn to the classification of graded central extensions of a groupoid $X$. In view of (2.11), it suffices to separately classify $\mathbb{T}$-central extensions (graded central extensions which are purely even) and gradings.

For the $\mathbb{T}$-central extensions, first recall that the category of $\mathbb{T}$-torsors on a space $Y$ is equivalent to the category whose objects are $\mathbb{T}$-valued Cech 1-cocycles, $\check{Z}^{1}(Y ; \mathbb{T})$, and in which a morphism from $z_{0}$ to $z_{1}$ is a Cech 0 -cochain $c \in \check{C}^{0}(Y ; \mathbb{T})$ with the property that

$$
\delta c=z_{1}-z_{0}
$$


Now consider the double complex for computing the Cech hyper-cohomology groups of the nerve $X_{\bullet}$, with coefficients in $\mathbb{T}$ :

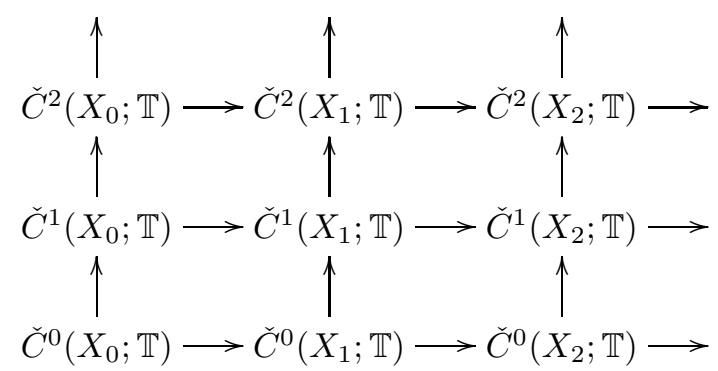

In terms of the Cech cocycle model for $\mathbb{T}$-bundles, the 2-category of $\mathbb{T}$-central extension of $X$ is equivalent to the category whose objects are cocycles in (2.12), of total degree 2 , whose component in $\check{C}^{2}\left(X_{0} ; \mathbb{T}\right)$ is zero. The 1 -morphisms are given by cochains of total degree 1 , whose coboundary has the property its component in $\check{C}^{2}\left(X_{0} ; \mathbb{T}\right)$ vanishes. The 2-morphisms are given by cochains of total degree 0 . Write

$$
\check{H}^{*}(X) \text { and } \check{H}^{*}\left(X_{0}\right)
$$

for the Cech hyper-cohomology of $X$, and the Cech cohomology of $X_{0}$ respectively. Then the group of isomorphism classes of even graded $\mathbb{T}$-gerbes is given by the kernel of the map

$$
\check{H}^{2}(X ; \mathbb{T}) \rightarrow \check{H}^{2}\left(X_{0} ; \mathbb{T}\right)
$$

the group of isomorphism classes of 1 -automorphisms of any even graded $\mathbb{T}$-gerbe is $\check{H}^{1}(X ; \mathbb{T})$, and the group of 2-automorphisms of any 1-morphism is $\check{H}^{0}(X ; \mathbb{T})$.

As for the gradings, the group of isomorphism classes of gradings is

$$
\operatorname{ker}\left\{\check{H}^{1}(X ; \mathbb{Z} / 2) \rightarrow \check{H}^{1}\left(X_{0} ; \mathbb{Z} / 2\right)\right\}
$$

and the automorphism group of any grading is

$$
\check{H}^{0}(X ; \mathbb{Z} / 2) \text {. }
$$

For convenience, write

$$
\check{H}_{\text {rel }}^{t}(X ; A)=\operatorname{ker}\left\{\check{H}^{t}(X ; A) \rightarrow \check{H}^{t}\left(X_{0} ; A\right)\right\} .
$$

Proposition 2.13. The group $\pi_{0} \underline{\mathfrak{E x t}}_{X}$ of isomorphism classes of graded central extension of $X$ is given by the set-theoretically split extension

$$
\check{H}_{\text {rel }}^{2}(X ; \mathbb{T}) \rightarrow \pi_{0} \underline{\mathfrak{E n t}}_{X} \rightarrow \check{H}_{\text {rel }}^{1}(X ; \mathbb{Z} / 2)
$$

with cocycle

$$
c(\epsilon, \mu)=\beta(\epsilon \cup \mu),
$$

where $\beta: \mathbb{Z} / 2=\{ \pm 1\} \subset \mathbb{T}$ is the inclusion. The group of isomorphism classes of automorphisms of any graded central extension of $X$ is $\check{H}^{1}(X ; \mathbb{T}) \times \check{H}^{0}(X ; \mathbb{Z} / 2)$, and the group of 2-automorphisms of any morphism of graded central extensions is $\check{H}^{0}(X ; \mathbb{T})$.

Proof: Most of this result was proved in the discussion leading up to its statement. The decomposition (2.11) gives the exact sequence, as well as a set-theoretic splitting

$$
s: \check{H}_{\mathrm{rel}}^{1}(X ; \mathbb{Z} / 2) \rightarrow \pi_{0} \underline{\mathfrak{E x t}}_{X} .
$$


It remains to identify the cocycle describing the group structure. Suppose that

$$
\epsilon, \mu: X \rightarrow \mathcal{B Z} / 2
$$

are two gradings of $X$. Then by the discussion leading up to (2.3), $s(\epsilon) s(\mu)$ is the graded central extension given by

$$
(s(\epsilon) s(\mu))_{f}=\mathbf{1}^{\epsilon(f)} \mathbf{1}^{\mu(f)} \approx \mathbf{1}^{\epsilon(f)+\mu(f)},
$$

and structure map

$$
\mathbf{1}^{\epsilon(g)} \mathbf{1}^{\mu(g)} \mathbf{1}^{\epsilon(f)} \mathbf{1}^{\mu(f)} \rightarrow \mathbf{1}^{\epsilon(g)} \mathbf{1}^{\epsilon(f)} \mathbf{1}^{\mu(g)} \mathbf{1}^{\mu(f)} \rightarrow \mathbf{1}^{\epsilon(g \circ f)} \mathbf{1}^{\mu(g \circ f)} .
$$

Using the canonical identifications

$$
\mathbf{1}^{a} \mathbf{1}^{b}=\mathbf{1}^{a+b}
$$

and

$$
\begin{aligned}
\epsilon(g \circ f) & =\epsilon(g)+\epsilon(f) \\
\mu(g \circ f) & =\mu(g)+\mu(f)
\end{aligned}
$$

one checks that (2.15) can be identified with the automorphism

$$
(-1)^{\epsilon(f) \mu(g)}
$$

of the trivialized graded line

$$
\mathbf{1}^{\epsilon(g)+\mu(g)+\epsilon(f)+\mu(f)} \approx \mathbf{1}^{\epsilon(g \circ f)+\mu(g \circ f)} .
$$

Similarly, the structure map of $s(\epsilon+\mu)$ can be identified with the identity map of the same trivialized graded line. It follows that $s(\epsilon) s(\mu)=c(\epsilon, \mu) s(\epsilon+\mu)$, where $c(\epsilon, \mu)$ is graded central extension with with $L_{f}=\mathbf{1}$, and

$$
\lambda_{g, f}=(-1)^{\epsilon(f) \mu(g)} .
$$

Now the 2-cocycle $\epsilon(f) \mu(g)$ is precisely the Alexander-Whitney formula for the cup product $\epsilon \cup \mu \in Z^{2}(X ; \mathbb{Z} / 2)$. This completes the proof.

One easy, but very useful consequence of Proposition 2.13 is the stacky nature of the morphism categories in $\underline{\mathfrak{E x t}}_{X}$.

Corollary 2.16. Let $P, Q \in \underline{\mathfrak{E x t}}_{X}$, and $f: Y \rightarrow X$ be a local equivalence. Then the functor

is an equivalence of categories, and so

$$
f^{*}: \underline{\mathfrak{E x t}}_{X}(P, Q) \rightarrow \underline{\mathfrak{E x t}}_{Y}\left(f^{*} P, f^{*} Q\right)
$$

$$
f^{*}: \mathfrak{E x t}_{X}(P, Q) \rightarrow \mathfrak{E x t}_{Y}\left(f^{*} P, f^{*} Q\right)
$$

is a bijection of sets.

Another useful, though somewhat technical consequence of the classification is

Corollary 2.17. Suppose that $X$ is a groupoid with the property that the maps

$$
\begin{aligned}
\check{H}^{2}(X ; \mathbb{T}) & \rightarrow \check{H}^{2}\left(X_{0} ; \mathbb{T}\right) \\
\check{H}^{1}(X ; \mathbb{Z} / 2) & \rightarrow \check{H}^{1}\left(X_{0} ; \mathbb{Z} / 2\right)
\end{aligned}
$$

are zero. If $Y \rightarrow X$ is a local equivalence, then the maps

$$
\begin{aligned}
\check{H}^{2}(Y ; \mathbb{T}) & \rightarrow \check{H}^{2}\left(Y_{0} ; \mathbb{T}\right) \\
\check{H}^{1}(Y ; \mathbb{Z} / 2) & \rightarrow \check{H}^{1}\left(Y_{0} ; \mathbb{Z} / 2\right)
\end{aligned}
$$


are zero, and

are equivalences.

$$
\underline{\mathfrak{E x t}}_{X} \rightarrow \underline{\mathfrak{E x t}}_{Y}, \text { and } \mathfrak{E x t}_{X} \rightarrow \mathfrak{E x t}_{Y}
$$

Proof: The first assertion is a simple diagram chase. It has the consequence that the maps

$$
\begin{aligned}
\check{H}_{\mathrm{rel}}^{2}(X ; \mathbb{T}) & \rightarrow \check{H}^{2}\left(X_{0} ; \mathbb{T}\right) \\
\check{H}_{\mathrm{rel}}^{1}(X ; \mathbb{Z} / 2) & \rightarrow \check{H}^{1}\left(X_{0} ; \mathbb{Z} / 2\right) \\
\check{H}_{\mathrm{rel}}^{2}(Y ; \mathbb{T}) & \rightarrow \check{H}^{2}\left(Y_{0} ; \mathbb{T}\right) \\
\check{H}_{\mathrm{rel}}^{1}(Y ; \mathbb{Z} / 2) & \rightarrow \check{H}^{1}\left(Y_{0} ; \mathbb{Z} / 2\right)
\end{aligned}
$$

are isomorphisms, and the second assertion follows.

For later reference we note the following additional consequence of Proposition 2.13

Corollary 2.18. Suppose that $X$ is a local quotient groupoid, and that $\tau$ is a twisting of $X$ represented by a local equivalence $P \rightarrow X$ and a graded central extension $\tilde{P} \rightarrow P$. Then $\tilde{P}$ is a local quotient groupoid.

Proof: Since the property of being a local quotient groupoid is an invariant of local equivalence, we know that $P$ is a local quotient groupoid. The question is also local in $P$, so we may assume $P$ is of the form $S / / G$ for a compact Lie group $G$. By our assumptions, the action of $G$ on $S$ has locally contractible slices. Working still more locally in $S$ we may assume $S$ is contractible. But then Proposition 2.13 implies that $\tau$ is given by a (graded) central extension of $\tilde{G} \rightarrow G$, and $\tilde{P}=S / / \tilde{G}$.

2.3. Twistings. We now describe the category $\mathfrak{T w i s t}_{X}$ of $K$-theory twistings on a local quotient groupoid $X(\Phi .2 .2)$. The objects of $\mathfrak{T w i s t}_{X}$ are pairs $a=(P, L)$ consisting of a local equivalence $P \rightarrow X$, and a graded central extension $L$ of $P$. The set of morphisms from $a=\left(P_{0}, L_{0}\right)$ to $b=\left(P_{1}, L_{1}\right)$ is defined to be the colimit

$$
\mathfrak{T}_{\mathfrak{w i s t}}(a, b)=\underset{p: P \rightarrow P_{12}}{\lim _{P}} \mathfrak{E x t}_{P}\left(p^{*} \pi_{1}^{*} a, p^{*} \pi_{2}^{*} b\right),
$$

where $P_{12}=P_{1} \times_{X} P_{2}$, the limit is taken over $\operatorname{Cov}_{P_{12}}$ and our notation refers to the diagram

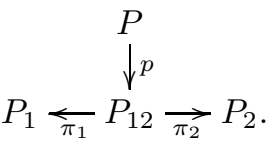

We leave to the reader to check that $\mathfrak{E x t}_{P}(a, b)$ does indeed define a functor on the 1-category quotient $\operatorname{Cov}_{P_{12}}$.

The colimit appearing in the definition of $\mathfrak{T w i s t}_{X}(a, b)$ is present in order that the definition be independent of any extraneous choices. In fact the colimit is attained at any stage.

Lemma 2.19. For any local equivalence

$$
p: P \rightarrow P_{12}
$$

the $\operatorname{map} \mathfrak{E x t}_{P}\left(p^{*} \pi_{1}^{*} a, p^{*} \pi_{2}^{*} b\right) \rightarrow \mathfrak{T}_{\mathfrak{w i s t}}(a, b)$ is an isomorphism. 
Proof: By Corollary 2.16, for any

$$
P^{\prime} \rightarrow P
$$

in $\operatorname{Cov}_{P_{12}}$, the map

$$
\mathfrak{E x t}_{P}(a, b) \rightarrow \mathfrak{E x t}_{P^{\prime}}(a, b)
$$

is an isomorphism. The result now follows from Corollary A.12,

For the composition law, suppose we are given three twistings

$$
a=\left(P_{1}, L_{1}\right), b=\left(P_{2}, L_{2}\right), \text { and } c=\left(P_{3}, L_{3}\right) .
$$

Find a $P_{123} \in \underline{\operatorname{Cov}}_{X}$ and maps

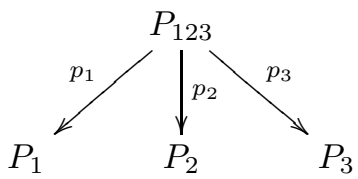

(for example one could take $P_{123}$ to be the (2-category) fiber product $P_{1} \times_{X} P_{2} \times_{X}$ $P_{3}$, and $p_{i}$ projection to the $i^{\text {th }}$ factor). By Lemma 2.19, the maps

$$
\begin{aligned}
& \mathfrak{E x t}_{P_{123}}\left(p_{1}^{*} a, p_{2}^{*} b\right) \rightarrow \mathfrak{T w i s t}_{X}(a, b) \\
& \mathfrak{E x t}_{P_{123}}\left(p_{1}^{*} a, p_{2}^{*} c\right) \rightarrow \mathfrak{T}_{\mathfrak{w i s t}}(a, c) \\
& \mathfrak{E x t}_{P_{123}}\left(p_{1}^{*} b, p_{2}^{*} c\right) \rightarrow \mathfrak{T w i s t}_{X}(b, c)
\end{aligned}
$$

are bijections. With these identifications, the composition law in $\mathfrak{T w i s t}_{X}$ is formed from that in $\mathfrak{E x t}_{P_{123}}$. We leave to the reader to check that this is well-defined.

The formation of $\mathfrak{T w i s t}_{X}$ is functorial in $X$. Given $f: Y \rightarrow X$, and $a=(P, L) \in$ $\mathfrak{T w i s t}_{X}$ form

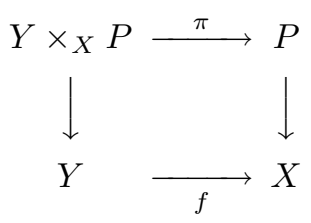

and set

$$
f^{*} a=\left(f^{*} P, \pi^{*} L\right) .
$$

Proposition 2.21. The association $X \mapsto \mathfrak{T w i s t}_{X}$ is a weak presheaf of groupoids. If $Y \rightarrow X$ is a local equivalence then $\mathfrak{T}_{\mathfrak{w i s t}} \rightarrow \mathfrak{T w i s t}_{X}$ is an equivalence of categories.

There is an evident functor

$$
\mathfrak{E x t}_{X} \rightarrow \mathfrak{T}_{\mathfrak{w i s t}}^{X}
$$

Proposition 2.22. When $X$ satisfies the condition of Corollary 2.17, the functor

$$
\mathfrak{E x t}_{X} \rightarrow \mathfrak{T w i s t}_{X}
$$

is an equivalence of categories.

Proof: Lemma 2.19 shows that (2.23) is fully faithful. Essential surjectivity is a consequence of Corollary 2.17. 
Corollary 2.24. If $Y \rightarrow X$ is a local equivalence, and $Y$ satisfies the conditions of Corollary 2.17 then the functors

$$
\mathfrak{T w i s t}_{X} \rightarrow \mathfrak{T}_{\mathfrak{w i s t}} \leftarrow \mathfrak{E x t}_{Y}
$$

are equivalences of groupoids.

Combining this with Proposition 2.13 gives

Corollary 2.25. The group $\pi_{0} \mathfrak{T w i s t}_{X}$ of isomorphism classes of twistings on $X$ is the set-theoretically split extension

$$
\check{H}^{2}(X ; \mathbb{T}) \rightarrow \pi_{0} \mathfrak{T}_{\mathfrak{w i s t}} \rightarrow \check{H}^{1}(X ; \mathbb{Z} / 2)
$$

with cocycle

$$
c(\epsilon, \mu)=\beta(\epsilon \cup \mu) .
$$

The group of automorphisms of any twisting is $\check{H}^{1}(X ; \mathbb{T}) \times \check{H}^{0}(X ; \mathbb{Z} / 2)$.

We now switch to the point of view of "fibered categories" in order to more easily describe the functorial properties of twisted $K$-groups.

Let $\mathfrak{E x t}$ denote the category whose objects are pairs $(X, L)$ consisting of a groupoid $X$ and a graded central extension $L$ of $X$. A morphism $(X, L) \rightarrow(Y, M)$ is a functor $f: X \rightarrow Y$, and an isomorphism $L \rightarrow f^{*} M$ in $\mathfrak{E x t}_{X}$. We identify morphisms

$$
f, g:(X, L) \rightarrow(Y, M)
$$

if there is a natural transformation $T: f \rightarrow g$ for which the following diagram commutes:

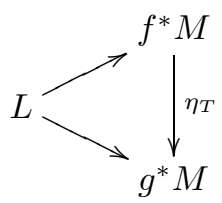

The functor $(X, L) \mapsto X$ from $\mathfrak{E} \mathfrak{x t}$ to groupoids represents $\mathfrak{E} \mathfrak{x t}$ as a fibered category, fibered over the category of groupoids.

Similarly, we define a category $\mathfrak{T} \mathfrak{w i s t}$ with objects $(X, a)$ consisting of a groupoid $X$, and a $K$-theory twisting $a \in \mathfrak{T}_{\mathfrak{w i s t}}$, and morphisms $(X, a) \rightarrow(Y, b)$ to be equivalence classes of pairs consisting of a functor $f: X \rightarrow Y$ and an isomorphism $a \rightarrow f^{*} b$ in $\mathfrak{T w i s t}_{X}$.

There is an inclusion $\mathfrak{E} \mathfrak{x t} \rightarrow \mathfrak{T} \mathfrak{w i s t}$ corresponding to the inclusion $\mathfrak{E x t}_{X} \rightarrow$ $\mathfrak{T}_{\mathfrak{w} i s t_{X}}$. Corollary 2.24 immediately implies

Lemma 2.26. Suppose that $F: \mathfrak{E x t} \rightarrow \mathcal{C}$ is a functor sending every morphism $(f, t):(X, L) \rightarrow(Y, M)$ in which $f$ is a local equivalence to an isomorphism. Then there is a factorization

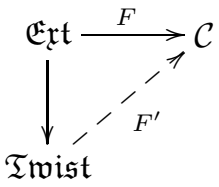

Moreover any two such factorizations are naturally isomorphic by a unique natural isomorphism. 


\subsection{Examples of twistings.}

Example 2.27. Suppose that $X$ is a space, $P \rightarrow X$ a principal $G$ bundle, and

$$
\begin{aligned}
& \tilde{G} \rightarrow G \\
& G \stackrel{\epsilon}{\rightarrow} \mathbb{Z} / 2
\end{aligned}
$$

is a graded central extension of $G$. Then $P / / G \rightarrow X$ is a local equivalence, $P / / \tilde{G}$ is a graded central extension of $P / / G$, and $(P / / \tilde{G}, P / / G)$ represents a twisting of $X$.

Example 2.28. As a special case, we note that any double cover $P \rightarrow X$ defines a twisting. In this case $G=\mathbb{Z} / 2, \tilde{G} \rightarrow G$ is the trivial bundle, and $\epsilon: G \rightarrow \mathbb{Z} / 2$ is the identity map. Any cohomology theory can be twisted by a double cover, and in fact these are the only twistings of ordinary cohomology with integer coefficients.

Example 2.29. Suppose that $X=\mathrm{pt} / / G$, with $G$ a compact Lie group. In this case every local equivalence $\tilde{X} \rightarrow X$ admits a section. Moreover the inclusion

$$
\left\{\operatorname{Id}_{X}\right\} \rightarrow \underline{\operatorname{Cov}}_{X}
$$

of the trivial category consisting of the identity map of $X$ into $\underline{\mathrm{Cov}}_{X}$ is an equivalence. It follows that

$$
\underline{\mathfrak{E x t}}\left(\mathrm{pt} / / G, \mathbb{T}^{ \pm}\right) \rightarrow \mathfrak{T w i s t}_{X}
$$

is an equivalence of categories, and so twistings of $X$ in this case are just graded central extensions of $G$. Using Corollary 2.25, one can draw the same conclusion for $S / / G$ when $S$ is contractible.

We now describe the main example of twistings used in this paper.

Example 2.30. Suppose that $G$ is a connected compact Lie group, and consider the path-loop fibration

$$
\Omega G \rightarrow P G \rightarrow G .
$$

We regard $P G$ as a principal bundle over $G$ with structure group $\Omega G$. The group $G$ acts on everything by conjugation. Write $L G$ for the group $\operatorname{Map}\left(S^{1}, G\right)$ of smooth maps from $S^{1}$ to $\mathrm{G}$. The homomorphism "evaluation at 1 " : $L G \rightarrow G$ is split by the inclusion of the constant loops. This exhibits $L G$ as a semidirect product

$$
L G \approx \Omega G \rtimes G .
$$

The group $L G$ acts on the fibration (2.31) by conjugation. The action of $L G$ on $G$ factors through the action of $G$ on itself by conjugation, through the map $L G \rightarrow G$. This defines a map of groupoids

$$
P G / / L G \rightarrow G / / G
$$

which is easily checked to be a local equivalence. A graded central extension $\tilde{L} G \rightarrow$ $L G$ then defines a twisting of $G / / G$.

We will write $\tau$ for a typical twisting of $X$, and write the monoidal structure additively: $\tau_{1}+\tau_{2}$. We will use the notations $\left(\tilde{P}^{\tau}, P^{\tau}\right)$ and $\left(L^{\tau}, P^{\tau}\right)$ for typical representing graded central extensions. This is consistent with writing the monoidal structure additively:

$$
L^{\tau_{1}+\tau_{2}}=L^{\tau_{1}} \otimes L^{\tau_{2}} .
$$


Example 2.32. Suppose that $Y=S / / G$ and $H \subset G$ is a normal subgroup. Write $X=S / / H$, and $f: X \rightarrow Y$ for the natural map. If $\tau$ is a twisting of $Y$ then $f^{*} \tau$ has a natural action of $G / H$ (in the 2-category sense), and the map $X \rightarrow Y$ is invariant under this action (again, in the 2-category sense). To see this it is easiest to replace $X$ by the weakly equivalent groupoid

$$
X^{\prime}=(S \times G / H) / / G
$$

and factor $X \rightarrow Y$ as

$$
X \stackrel{i}{\rightarrow} X^{\prime} \stackrel{f^{\prime}}{\rightarrow} Y .
$$

Since $i^{*}: \mathfrak{T w i s t}_{X^{\prime}} \rightarrow \mathfrak{T w i s t}_{X}$ is an equivalence of 2-categories, it suffices to exhibit an action of $G / H$ on ${f^{\prime *}}^{*}$. The obvious left action of $G / H$ on $S \times G / H$ commutes with the right action of $G$, giving an action of $G / H$ on $X^{\prime}$ commuting with $f^{\prime}$. The action of $G / H$ on $f^{\prime *} \tau$ is then a consequence of naturality.

Example 2.33. By way of illustration, consider the situation of Example 2.32 in which $H$ is commutative, $S=\{\mathrm{pt}\}$, and $\tau$ is given by a central extension

$$
\mathbb{T} \mapsto \tilde{G} \rightarrow G .
$$

Write

$$
\mathbb{T} \mapsto \tilde{H} \rightarrow H,
$$

for the restriction of $\tau$ to $H$ and assume, in addition, that $\tilde{H}$ is commutative. Then the action of $G / H$ on $f^{*} \tau$ constructed in Example 2.32 works out to be the natural action of $G / H$ on $\tilde{H}$ given by conjugation.

\section{TWISTED K-GROUPS}

3.1. Axioms. Before turning to the definition of twisted $K$-theory, we list some general properties describing it as a cohomology theory on the category $\mathfrak{T} \mathfrak{w i s t}$ of local quotient groupoids equipped with a twisting. These properties almost uniquely determine twisted $K$-theory, and suffice to make our main computation in Section 4 .

Twisted $K$-theory is going to be homotopy invariant, so we need to define the notion of homotopy

Definition 3.1. A homotopy between two maps

$$
f, g:\left(X, \tau_{X}\right) \rightarrow\left(Y, \tau_{Y}\right)
$$

is a map

$$
\left(X \times[0,1], \pi^{*} \tau_{X}\right) \rightarrow\left(Y, \tau_{Y}\right)
$$

$(\pi: X \times[0,1] \rightarrow X$ is the projection) whose restriction to $X \times\{0\}$ is $f$, and to $X \times\{1\}$ is $g$.

Twisted $K$-theory is also a cohomology theory. To state this properly involves defining the relative twisted $K$ theory of a triple $(X, A, \tau)$ consisting of a local quotient groupoid $X$, a sub-groupoid $A$, and a twisting of $X$. We form a category of the triples $(X, A, \tau)$ in the same way we formed $\mathfrak{T}$ wist. We'll call this the category of pairs in $\mathfrak{T} \mathfrak{w i s t}$.

We now turn to the axiomatic properties of twisted $K$-theory.

Proposition 3.2. The association $(X, A, \tau) \mapsto K^{\tau+n}(X, A)$ to be constructed in 3.4 is a contravariant homotopy functor on the category of pairs $(X, A, \tau)$ in $\mathfrak{T w i s t}$, taking local equivalences to isomorphisms. 
Proposition 3.3. The functors $K^{\tau+n}$ form a cohomology theory:

i) there is a natural long exact sequence

$$
\begin{aligned}
\cdots \rightarrow K^{\tau+n} & (X, A) \rightarrow K^{\tau+n}(X) \rightarrow K^{\tau+n}(A) \\
& \rightarrow K^{\tau+n+1}(X, A) \rightarrow K^{\tau+n+1}(X) \rightarrow K^{\tau+n+1}(A) \rightarrow \cdots .
\end{aligned}
$$

ii) If $Z \subset A$ is a (full) subgroupoid whose closure is contained in the interior of $A$, then the restriction (excision) map

$$
K^{\tau+n}(X, A) \rightarrow K^{\tau+n}(X \backslash Z, A \backslash Z)
$$

is an isomorphism.

iii) If $(X, A, \tau)=\coprod_{\alpha}\left(X_{\alpha}, A_{\alpha}, \tau_{\alpha}\right)$, then the natural map

$$
K^{\tau+n}(X, A) \rightarrow \prod_{\alpha} K^{\tau_{\alpha}+n}\left(X_{\alpha}, A_{\alpha}\right)
$$

is an isomorphism.

The combination of excision and the long exact sequence of a pair gives the Mayer-Vietoris sequence

$$
\begin{aligned}
\ldots & \rightarrow K^{\tau+n}(X) \rightarrow K^{\tau+n}(U) \oplus K^{\tau+n}(V) \rightarrow K^{\tau+n}(U \cap V) \\
& \rightarrow K^{\tau+n+1}(X) \rightarrow \cdots
\end{aligned}
$$

when $X$ is written as the union of two sub-groupoids whose interiors form a covering.

Proposition 3.4. $\quad$ i) There is a bilinear pairing

$$
K^{\tau+n}(X) \otimes K^{\mu+m}(X) \rightarrow K^{\tau+\mu+n+m}(X)
$$

which is associative and (graded) commutative up to the natural isomorphisms of twistings coming from Proposition 3.2.

ii) Suppose that $\eta: \tau \rightarrow \tau$ is a 1-morphism, corresponding to a graded line bundle $L$ on $X$. Then

$$
\eta_{*}=\text { multiplication by } L: K^{\tau+n}(X) \rightarrow K^{\tau+n}(X)
$$

where $L$ is regarded as an element of $K^{0}(X)$ and the multiplication is that of (1)

Twisted $K$-theory also reduces to equivariant $K$-theory in special cases.

Proposition 3.5. Let $X=S / / G$ be a global quotient groupoid, with $G$ a compact Lie group, and $\tau$ a twisting given by a graded central extension

$$
\begin{aligned}
& \mathbb{T} \rightarrow G^{\tau} \rightarrow G \\
& \epsilon: G \rightarrow \mathbb{Z} / 2 .
\end{aligned}
$$

i) If $\epsilon=0$ then then $K^{\tau+n}(X)$ is the summand

$$
K_{G^{\tau}}^{n}(S)(1) \subset K_{G^{\tau}}^{n}(S)
$$

on which $\mathbb{T}$ acts via its standard (defining) representation. This isomorphism is compatible with the product structure. 
ii) For general $\epsilon, K^{\tau+n}(X)$ is isomorphic to

$$
K_{G^{\tau}}^{n+1}(S \times(\mathbb{R}(\epsilon), \mathbb{R}(\epsilon) \backslash\{0\}))(1),
$$

in which the symbol $\mathbb{R}(\epsilon)$ denotes the 1-dimensional representation $(-1)^{\epsilon}$ of $G^{\tau}$.

In part ii), When $\epsilon=0$, then $\mathbb{R}(\epsilon)$ is the trivial representation, and the isomorphism can be composed with the suspension isomorphism to give the isomorphism of i).

When $\tau=0$, so that $G^{\tau} \approx G \times \mathbb{T}$, Proposition 3.5 reduces to an isomorphism

$$
K^{\tau+n}(X) \approx K_{G}^{n}(S) .
$$

In view of this, we'll often write

$$
K_{G}^{\tau+n}(S)=K^{\tau+n}(X)
$$

in case $X=S / / G$ and $\epsilon=0$. Of course there is also a relative version of Proposition 3.5.

The reader is referred to Section 4 of 21 for a more in depth discussion of the twistings of equivariant $K$-theory, and interpretation of " $\epsilon$ " part the twisting in terms of graded representations.

Using the Mayer-Vietoris sequence one can easily check that result of part i) of Proposition 3.5 holds for any local quotient groupoid $X$. If the twisting $\tau$ is represented by a central extension $P \rightarrow X$, then the restriction mapping is an isomorphism

$$
K^{\tau+n}(X) \approx K^{n}(P)(1) .
$$

In this way, once $K$-theory is defined for groupoids, twisted $K$-theory is also defined.

3.2. Twisted Hilbert spaces. Our definition of twisted $K$-theory will be in terms of Fredholm operators on a twisted bundle of Hilbert spaces. In this section we describe how one associates to a graded central extension of a groupoid, a twisted notion of Hilbert bundle. We refer the reader to Appendix A, A.4 for our notation and conventions on bundles over groupoids, and to $\mathrm{A} .4$ for a discussion of Hilbert space bundles.

Let $X$ be a groupoid, and $\tau: \tilde{X} \rightarrow X$ a graded central extension, whose associated graded $\mathbb{T}$-bundle we denote $L^{\tau} \rightarrow X_{1}$. As in Appendix A.3, we will use

$$
a \stackrel{f}{\rightarrow} b \quad \text { and } \quad a \stackrel{f}{\rightarrow} b \stackrel{g}{\rightarrow} c
$$

to refer to generic points of $X_{1}$ and $X_{2}$ respectively, and so, for example, in a context describing bundles over $X_{2}$, the symbol $H_{b}$ will refer to the pullback of $X$ along the map

$$
\begin{aligned}
X_{2} & \rightarrow X_{0} \\
(a \rightarrow b & \rightarrow c) \mapsto b .
\end{aligned}
$$

Definition 3.6. A $\tau$-twisted Hilbert bundle on $X$ consists of a $\mathbb{Z} / 2$-graded Hilbert bundle $H$ on $X_{0}$, together with an isomorphism (on $X_{1}$ )

$$
L_{f}^{\tau} \otimes H_{a} \rightarrow H_{b}
$$


satisfying the cocycle condition that

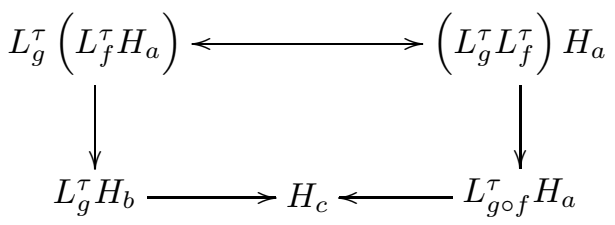

commutes on $X_{2}$.

Remark 3.7. Phrased differently, a twisted Hilbert bundle is just a graded Hilbert bundle over $\tilde{X}$, with the property that the map $H_{a} \rightarrow H_{b}$ induced by $(a \stackrel{f}{\rightarrow} b) \in \tilde{X}_{1}$ has degree $\epsilon(f)$, and for which the central $\mathbb{T}$ acts according to its defining character.

Example 3.8. Suppose that $X$ is of the form $P / / G$, and that our twisting corresponds to a central extension of $G^{\tau} \rightarrow G$. Then a projective unitary representation of $G$ (meaning a representation of $G^{\tau}$ on which the central $\mathbb{T}$ acts according to its defining character) defines a twisted Hilbert bundle over $X$.

Suppose that $\tau$ and $\mu$ are graded central extensions of $X$, with associated graded $\mathbb{T}$-torsors $L^{\tau}$ and $L^{\mu}$. If $H$ is a $\tau$-twisted Hilbert bundle over $X$ and $W$ is a $\mu$-twisted Hilbert bundle, then the graded tensor product $H \otimes W$ is a $(\tau+\mu)$-twisted Hilbert bundle, with structure map

$$
L_{f}^{\tau+\mu} \otimes H_{a} \otimes W_{a}=L_{f}^{\tau} \otimes L_{f}^{\mu} \otimes H_{a} \otimes W_{a} \rightarrow L_{f}^{\tau} \otimes H_{a} \otimes L_{f}^{\mu} \otimes W_{a} \rightarrow H_{b} \otimes W_{b} .
$$

Now suppose that $H^{1}$ and $H^{2}$ are $\tau$-twisted, graded Hilbert bundles over $X$.

Definition 3.9. A linear transformation $T: H^{1} \rightarrow H^{2}$ consists of a linear transformation of Hilbert bundles $T: H^{1} \rightarrow H^{2}$ on $X_{0}$ for which the following diagram commutes on $X_{1}$ :

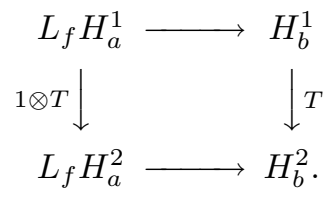

If $L^{\tau}$ is a graded central extension of $X$, we'll write $\mathcal{U}_{X}^{\tau}$ (or just $\mathcal{U}^{\tau}$ if $X$ is understood) for the category in which the objects are $\tau$-twisted $Z / 2$-graded Hilbert bundles, and with morphisms the linear isometric embeddings. If $f: Y \rightarrow X$ is a map, there is an evident functor

$$
f^{*}: \mathcal{U}_{X}^{\tau} \rightarrow \mathcal{U}_{Y}^{f^{*} \tau}
$$

A natural transformation $T: f \rightarrow g$ of functors $X \rightarrow Y$ gives a natural transformation $T^{*}: f^{*} \rightarrow g^{*}$. Using Remark 3.7 and descent, one easily checks that $f^{*}$ is an equivalence of categories when $f$ is a local equivalence.

The category $\mathcal{U}_{X}^{\tau}$ is also functorial in $\tau$. Indeed, suppose that $\eta: \tau \rightarrow \sigma$ is a morphism, given by a graded $\mathbb{T}$-bundle $\eta$, and an isomorphism

$$
\eta_{b} \otimes \tau_{f} \rightarrow \sigma_{f} \otimes \eta_{a}
$$

If $H$ is a $\tau$-twisted Hilbert bundle, then $H \otimes \eta$ is a $\sigma$-twisted Hilbert bundle. One easily checks that $H \mapsto H \otimes \eta$ gives an equivalence of categories $\mathcal{U}_{X}^{\tau} \rightarrow \mathcal{U}_{X}^{\sigma}$, with inverse $H \mapsto H \otimes \eta^{-1}$. The 2-morphisms $\eta_{1} \rightarrow \eta_{2}$ give natural isomorphisms of functors. 
The tensor product of Hilbert spaces gives a natural tensor product

$$
\mathcal{U}_{X}^{\tau} \times \mathcal{U}_{X}^{\mu} \rightarrow \mathcal{U}_{X}^{\tau+\mu}
$$

3.3. Universal Twisted Hilbert Bundles. We now turn to the existence of special kinds of $\tau$-twisted Hilbert bundles, following the discussion of $\$$ A.4. We keep the notation of 3.2

Definition 3.10. A $\tau$-twisted Hilbert bundle $H$ on $X$ is

i) universal if for every $\tau$-twisted Hilbert space bundle $V$ there is a unitary embedding $V \rightarrow H$;

ii) locally universal if $\left.H\right|_{U}$ is universal for every open sub-groupoid $U \subset X$;

iii) absorbing if for every $\tau$-twisted Hilbert space bundle $V$ there is an isomorphism $H \oplus V \approx H$;

iv) locally absorbing if $\left.H\right|_{U}$ is absorbing for every open sub-groupoid $U \subset X$.

As in Appendix A.4, if $H$ is (locally) universal, then $H$ is automatically absorbing.

Lemma 3.11. Suppose that $\tilde{X} \rightarrow X$ is a graded central extension and $H$ is a graded Hilbert bundle on $\tilde{X}$. Let $H(1) \subset H$ be the eigenbundle on which the central $\mathbb{T}$ acts according to its defining representation. Then $H(1)$ is a $\tau$-twisted Hilbert bundle on $X$ which is (locally) universal if $H$ is.

Lemma 3.12. If $X$ is a local quotient groupoid, and $\tau: \tilde{X} \rightarrow X$ is a graded central extension then there exists a locally universal $\tau$-twisted Hilbert bundle $H$ on $X$. The bundle $H$ is unique up to unitary equivalence.

Proof: By Corollary 2.18, $\tilde{X}$ is a local quotient groupoid which, by Corollary A.33, admits a locally universal Hilbert bundle. The result now follows from Lemma 3.11

3.4. Definition of twisted $K$-groups. Our task is to define twisted $K$-groups for pairs $(X, A, \tau)$ in Twist. In view of Lemma 2.26 it suffices to define functors $K^{\tau+*}(X, A)$ for $(X, A, \tau)$ in $\mathfrak{E} \mathfrak{x t}$, and show that they take local equivalences to isomorphisms. We will do this by using spaces of Fredholm operators to construct a spectrum $\underline{K}^{\tau}(X, A)$ and defining $K^{\tau+n}(X, A)=\pi_{-n} \underline{K}^{\tau}(X, A)$. The reader is referred to $\$$ A.5 for some background discussion on spaces of Fredholm operators.

Suppose then that $(X, \tau)$ is an object of $\mathfrak{E x t}$ and $H$ is a locally universal, $\tau$ twisted Hilbert bundle over $X$. With the notation of 3.2 is given by a Hilbert bundle $H$ over $X_{0}$, equipped with an isomorphism

$$
L_{f}^{\tau} \otimes H_{a} \rightarrow H_{b}
$$

over $f: a \rightarrow b \in X_{1}$, satisfying the cocycle condition. The map

$$
T \mapsto \mathrm{Id} \otimes T
$$

is a homeomorphism between the spaces of Fredholm operators (See A.5) Fred ${ }^{(n)}\left(H_{a}\right)$ and $\operatorname{Fred}^{(n)}\left(L_{f}^{\tau} \otimes H_{a}\right)$ compatible with the structure maps (3.13). The spaces Fred $^{(n)}\left(H_{a}\right)$ therefore form a fiber bundle over $\operatorname{Fred}^{(n)}(H)$ over $X$. 
We define spaces $\underline{K}^{\tau}(X)_{n}$ by

$$
\underline{K}^{\tau}(X)_{n}= \begin{cases}\Gamma\left(X ; \text { Fred }^{(0)}(H)\right) & n \text { even } \\ \Gamma\left(X ; \operatorname{Fred}^{(1)}(H)\right) & n \text { odd },\end{cases}
$$

By an obvious modification of the arguments of Atiyah-Singer [1], the results described in $\$$ A.5 hold for the bundle $\operatorname{Fred}^{(n)}(H)$ over $X$. In particular, the maps (A.44) and the homeomorphism A.45 give weak homotopy equivalences

$$
\underline{K}^{\tau}(X)_{n} \rightarrow \Omega \underline{K}^{\tau}(X)_{n+1},
$$

making the collection of spaces

$$
\underline{K}^{\tau}(X)=\left\{\underline{K}^{\tau}(X)_{n}\right\}
$$

into a spectrum.

Definition 3.14. Suppose that $(X, \tau)$ is a local quotient groupoid equipped with a graded central extension $\tau$, and $H$ is a locally universal, $\tau$-twisted Hilbert bundle over $X$. The twisted $K$-theory spectrum of $X$ is the spectrum $\underline{K}^{\tau}(X)$ defined above.

To keep things simple, we do not indicate the choice of Hilbert bundle $H$ in the notation $\underline{K}^{\tau}(X)$. The value of the twisted $K$-group is, in the end, independent of this choice. See Remark 3.17 below.

We now turn to the functorial properties of $X \mapsto \underline{K}^{\tau}(X)$. Suppose that $f: Y \rightarrow$ $X$ is a map of local quotient groupoids, and $\tau$ is a twisting of $X$. Let $H_{X}$ be a $\tau$-twisted, locally universal Hilbert bundle over $X$, and $H_{Y}$ an $f^{*} \tau$-twisted, locally universal Hilbert space bundle over $Y$. Since $H_{Y}$ is universal, there is a unitary embedding $f^{*} H_{X} \subset H_{Y}$. Pick one. There is then an induced map

$$
\begin{aligned}
f^{*} \operatorname{Fred}^{(n)}\left(H_{X}\right) & \rightarrow \text { Fred }^{(n)}\left(H_{Y}\right) \\
T & \mapsto T \oplus \epsilon,
\end{aligned}
$$

( $\epsilon$ is the base point) and so a map of spectra

$$
f^{*}: \underline{K}^{\tau}(X) \rightarrow \underline{K}^{\tau}(Y) .
$$

Suppose that $\eta: \sigma \rightarrow \tau$ is a morphism of central extensions of $X$, given by a graded $T$-bundle $\eta$ over $X_{0}$, and isomorphism

$$
\eta_{b} \otimes \sigma_{f} \rightarrow \tau_{f} \otimes \eta_{a}
$$

If $H$ is a locally universal $\sigma$-twisted Hilbert bundle, then $H \otimes \eta$ is a locally universal $\tau$-twisted Hilbert bundle. The map

$$
T \mapsto T \otimes \operatorname{Id}_{\eta}
$$

then gives a homeomorphism $\operatorname{Fred}^{(n)}(H) \rightarrow \operatorname{Fred}^{(n)}(H \otimes \eta)$, and so an isomorphism of spectra

$$
\eta_{*}: \underline{K}^{\sigma}(X) \rightarrow \underline{K}^{\tau}(X)
$$

Since automorphisms of $\eta$ commute with the identity map, 2-morphisms of twistings have no effect on $\eta_{*}$. In this way the association $\tau \mapsto K^{\tau}(X)$ can be made into a functor on $\mathfrak{E x t}_{X}$.

Now we come to an important point. Suppose $Y \rightarrow X$ is the inclusion of a (full) subgroupoid of a local quotient groupoid, and $H_{X}$ is locally universal. By Corollary A.34, we may then take $H_{Y}$ to be $f^{*} H_{X}$. The bundle of spectra $\underline{K}^{\tau}(Y)$ is then just the restriction of $\underline{K}^{\tau}(X)$. This would not be true for general groupoids and 
is the reason for our restriction to local quotient groupoids. We use this restriction property in the definition of the twisted $K$-theory of a pair. While this could be avoided, the restriction property plays a key role in the proof of excision, and does not appear to be easily avoided there.

Definition 3.15. Suppose that $A \subset X$ is a sub-groupoid of a local quotient groupoid, and that $\tau$ is a graded central extension of $X$. The twisted $K$-theory spectrum of $(X, A, \tau)$ is the homotopy fiber $\underline{K}^{\tau}(X, A)$ of the restriction map $\underline{K}^{\tau}(X) \rightarrow$ $\underline{K}^{\tau}(A)$.

If we write

$$
\Gamma\left(X, A ; \operatorname{Fred}^{(n)}(H)\right) \subset \Gamma\left(X ; \operatorname{Fred}^{(n)}(H)\right)
$$

for the subspace of sections whose restriction to $A$ is the basepoint $\epsilon$, then

$$
\underline{K}^{\tau}(X, A)_{n}=\Gamma\left(N, A ; \operatorname{Fred}^{(n)}(H)\right),
$$

where $N$ is the mapping cylinder of $A \subset X$

$$
N=X \amalg A \times[0,1] / \sim .
$$

Definition 3.16. The twisted $K$-group $K^{\tau+n}(X, A)$ is the group $\pi_{-n} \underline{K}^{\tau}(X, A)=$ $\pi_{0} \underline{K}^{\tau}(X, A)_{n}$.

Remark 3.17. There are several unspecified choices that go into the definition of the spectra $\underline{K}^{\tau}(X, A)$, and the induced maps between them as $X, A$ and $\tau$ vary. It follows from Propositions A.35 and A.36 that these choices are parameterized by (weakly) contractible spaces, and so have no effect on the homotopy invariants (such as twisted $K$-groups, and maps of twisted $K$-groups) derived from them.

\subsection{Verification of the axioms.}

3.5.1. Proof of Proposition [3.2: functoriality. Most of this result was proved in the process of defining the groups $K^{\tau+n}(X, A)$. Functoriality in Ext follows from the discussion of 33.4 and Remark 3.17. For homotopy invariance, note that if $H$ is a locally universal $\tau$-twisted Hilbert bundle over $(X, A)$, then $\pi^{*} H$ is a locally universal Hilbert space bundle over $(X, A) \times I$, and so

$$
\underline{K}_{n}^{\tau}((X, A) \times I)=\underline{K}_{n}^{\tau}(X, A)^{I},
$$

and the two restriction maps to $\underline{K}_{n}^{\tau}(X)$ correspond to evaluation of paths at the two endpoints. The two restriction maps are thus homotopic, and homotopy invariance follows easily.

The assertion about local equivalences is an immediate consequence of descent. As remarked at the beginning of 33.4 , this, in turn, gives functoriality on the category of pairs in $\mathfrak{T}$ wist.

3.5.2. Proof of Proposition 3.3; cohomological properties. The long exact sequence of a pair (assertion i)is just the long exact sequence in homotopy groups associated to the fibration of spectra

$$
\underline{K}^{\tau}(X, A) \rightarrow \underline{K}^{\tau}(X) \rightarrow \underline{K}^{\tau}(A),
$$

The "wedge axiom" (part iii) is immediate from the definition. More significant is excision (part ii). In describing the proof, we will freely use, in the context of 
groupoids, the basic constructions of homotopy theory as described in A.2.1. Write $U=X \backslash Z$ and let $N$ be the double mapping cylinder of

$$
U \leftarrow U \cap A \rightarrow A .
$$

Then the map $N \rightarrow X$ is a homotopy equivalence of groupoids, and so by the diagram of fibrations

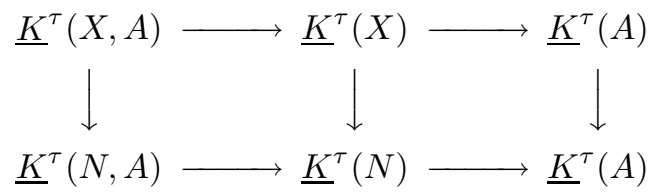

the map

$$
\underline{K}^{\tau}(X, A) \rightarrow \underline{K}^{\tau}(N, A)
$$

is a weak equivalence. Similarly, if $N^{\prime}$ denote the mapping cylinder of $U \cap A \rightarrow U$, then

$$
\underline{K}^{\tau}(X \backslash Z, A \backslash Z)=\underline{K}^{\tau}(U, U \cap A) \rightarrow \underline{K}^{\tau}\left(N^{\prime}, U \cap A\right)
$$

is a weak equivalence. We therefore need to show that for each $n$, the map

$$
\underline{K}^{\tau}(N, A)_{n} \rightarrow \underline{K}^{\tau}\left(N^{\prime}, U \cap A\right)_{n}
$$

is a weak equivalence. Let $H$ be a locally universal $\tau$-twisted Hilbert bundle over $X$. Then the pullback of $H$ to each of the (local quotient) groupoids $N, N^{\prime} A, U$, $U^{\prime}, U \cap A$ is also locally universal. It follows that the twisted $K$-theory spectra of each of these groupoids is defined in terms of sections of the bundle pulled back from Fred $^{(n)}\left(H \otimes C_{1}\right)$. To simplify the notation a little, let's denote all of these pulled back bundles Fred ${ }^{(n)}$. Now consider the diagram

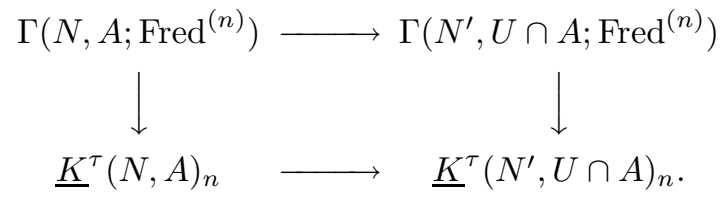

We are to show that the bottom row is a weak equivalence. But the top row is a homeomorphism, and the vertical arrows are weak equivalences since the maps

$$
\begin{aligned}
(N \cup \operatorname{cyl}(A), A) & \rightarrow(N, A) \\
\left(N^{\prime} \cup \operatorname{cyl}(U \cap A), U \cap A\right) & \rightarrow\left(N^{\prime}, U \cap A\right)
\end{aligned}
$$

are relative homotopy equivalences.

3.5.3. Proof of Proposition 3.4: Multiplication. The multiplication is derived from the pairing

$$
\begin{aligned}
\operatorname{Fred}^{(n)}\left(H_{1}\right) \times \operatorname{Fred}^{(m)}\left(H_{2}\right) & \rightarrow \text { Fred }^{(n+m)}\left(H_{1} \otimes H_{2}\right) \\
(S, T) & \mapsto S * T=S \otimes \operatorname{Id}+\operatorname{Id} \otimes T,
\end{aligned}
$$

the tensor structure on the category of twisted Hilbert space bundles described in 93.2 and the natural identification of the $\mathbb{Z} / 2$-graded tensor product $\mathrm{C} \ell\left(\mathbb{R}^{n}\right) \otimes$ $\mathrm{C} \ell\left(\mathbb{R}^{m}\right) \approx \mathrm{C} \ell\left(R^{n+m}\right.$ Verification of part i $)$ is left to the reader.

Even if one of $S, T$ is not acting on a locally universal Hilbert bundle the product $S * T$ will. This is particularly useful when describing the product of an element of untwisted $K$-theory, with one of twisted $K$-theory. For example if $V$ is a vector bundle over $X$, we can choose a Hermitian metric on $V$, regard $V$ as a bundle of 
finite dimensional graded Hilbert spaces, with odd component 0 , and take $S=0$. Then $S * T$ is just the identity map of $V$ tensored with $T$. More generally, a virtual difference $V-W$ of $K^{0}(X)$ can be represented by the odd, skew-adjoint Fredholm operator $S=0$ on the graded Hilbert space whose even part is $V$ and whose odd part is $W$, and $S * T$ represents the product of $V-W$ with the class represented by $T$. The assertion of Part ii) is the special case in which $V$ is a graded line bundle.

3.5.4. Proof of Proposition 3.5: Equivariant $K$-theory. Let $X=S / / G$ be a global quotient, and $\tau$ a twisting given by a graded central extension $G^{\tau}$ of $G$, and a homomorphism $\epsilon: G \rightarrow \mathbb{Z} / 2$. Replacing $X$ with $X \times(\mathbb{R}(\epsilon), \mathbb{R}(\epsilon) \backslash\{0\})$ and using (3.19), if necessary, we may reduce to the case $\epsilon=0$. Write $V(1)$ for the summand of

$$
V=C_{1} \otimes L^{2}\left(G^{\tau}\right) \otimes \ell^{2}
$$

on which the central $\mathbb{T}$ acts according to its defining character. Then $H=S \times V(1)$ is a locally universal Hilbert bundle. Our definition of $K^{\tau}(X)$ becomes

$$
K^{\tau-n}(X)=\left[S, \text { Fred }^{(n)}\left(C_{n} \otimes V(1)\right)\right]^{G}
$$

which is the summand of

$$
\left[S, \text { Fred }^{(n)}\left(C_{n} \otimes V\right)\right]^{G^{\tau}}
$$

corresponding to the defining representation of $\mathbb{T}$. So the result follows from the fact that $\operatorname{Fred}^{(n)}\left(C_{n} \otimes V\right)$ is a classifying space for $K_{G^{\tau}}^{-n}$. While this is certainly wellknown, we were unable to find an explicit statement in the literature. It follows easily from the case in which $G$ is trivial. Indeed, the universal index bundle is classified by a map to any classifying space for equivariant $K$-theory, and it suffices to show that this map is a weak equivalence on the fixed point spaces for the closed subgroups $H$ of $G$. The assertion for the fixed point spaces easily reduces to the main result of [1].

3.6. The Thom isomorphism, pushforward, and the Pontryagin product. We begin with a general discussion. Let $E=\left\{E_{n} \stackrel{t_{n}}{\longrightarrow} \Omega E_{n+1}\right\}_{n=0}^{\infty}$ be a spectrum. For a real vector space $V$, equipped with a positive definite metric let $\Omega^{V}\left(E_{n}\right)$ denote the space of maps from the unit ball $B(V)$ to $E_{n}$, sending the unit sphere $S(V)$ to the base point. The collection of spaces $\Omega^{V} E_{n}$ forms a spectrum $\Omega^{V} E$. An isomorphism $V \approx \mathbb{R}^{k}$ gives an identification $\Omega^{V} E_{n} \approx E_{n-k}$, and of $\Omega^{V} E$ with the spectrum derived from $E$ by simply shifting the indices. Such a spectrum is called a "shift desuspension" of $E$ (see [25). Some careful organization is required to avoid encountering signs by moving loop coordinates past each other. The reader is referred to 25] for more details. Of course, for a space $X$ one has

$$
\left(\Omega^{V} E\right)^{n}(X) \approx E^{n}(X \times(V, V \backslash\{0\})) \approx E^{-k+n}(X) .
$$

Now suppose that $V$ is a vector bundle of dimension $k$ over a space $X$. The construction described above can be formed fiberwise to form a bundle

$$
\Omega^{V} E=\left\{\Omega^{V} E_{n}\right\}
$$

of spectra over $X$. The group of vertical homotopy classes of sections

$$
\pi_{0} \Gamma\left(X, \Omega^{V} E_{n}\right)
$$

can then be thought of as a twisted form of $\left[X, E_{-k+n}\right]=E^{-k+n}(X)$. We denote this twisted (generalized) cohomology group

$$
E^{-\tau_{V}+n}(X) .
$$


Now the group (3.18) is the group of pointed homotopy classes of maps $\left[X^{V}, E_{n}\right]$ from the Thom complex of $V$ to $E_{n}$. This gives a tautological Thom isomorphism

$$
\tilde{E}^{n}\left(X^{V}\right)=E^{n}(B(V), S(V)) \approx \tilde{E}^{-\tau_{V}+n}(X) .
$$

The more usual Thom isomorphisms arise when a geometric construction is used to trivialize the bundle $\Omega^{V} E$. Such a trivialization is usually called an " $E$-orientation of $V$."

We now return to the case $E=K$, with the aim of identifying the twisting $\tau_{V}$ with type defined in $₫ 2$. The main point is that the action of the orthogonal group $O(k)$ on $\Omega^{k}$ Fred $^{(n)}$ lifts through the Atiyah-Singer map Fred ${ }^{(k+n)} \rightarrow \Omega^{k}$ Fred $^{(n)}$. Our discussion of this matter is inspired by the Stoltz-Teichner 31 description of Spin-structures, and, of course Donovan-Karoubi [14].

Let $X$ be a local quotient groupoid, and $V$ a real vector bundle over $X$ of dimension $k$, and $\mathrm{C} \ell(V)$ the associated bundle of Clifford algebras. The bundle $\mathrm{C} \ell(V) \otimes H$ is a locally universal $\mathrm{C} \ell(V)$-module. The Atiyah-Singer construction [1] gives a map

$$
\operatorname{Fred}_{\mathrm{C} \ell(V)}(\mathrm{C} \ell(V) \otimes H) \rightarrow \Omega^{V} \operatorname{Fred}^{(0)}(\mathrm{C} \ell(V) \otimes H)
$$

which is a weak equivalence on global sections. We can therefore trivialize the bundle of spectra $\Omega^{V} K$ by trivializing the bundle of Clifford algebras $\mathrm{C} \ell(V)$.

Of course something weaker will also trivialize $\Omega^{V} K$. We don't really need a bundle isomorphism $\mathrm{C} \ell(V) \approx X \times C_{k}$. We just need a way of going back and forth between $\mathrm{C} \ell(V)$-modules and $C_{k}$ modules. It is enough to have a bundle of irreducible $\mathrm{C} \ell(V)-C_{k}$ bimodules giving a Morita equivalence.

Let $M=C_{k}$, regarded as a $\mathrm{C} \ell\left(\mathbb{R}^{k}\right)-C_{k}$-bimodule. We equip $M$ with the Hermitian metric in which the monomials in the $\epsilon_{i}$ are orthonormal. Consider the group $\operatorname{Pin}^{\mathrm{c}}(k)$ of pairs $(t, f)$ in which $t: \mathbb{R}^{k} \rightarrow \mathbb{R}^{k}$ is an orthogonal map, and

$$
f: t^{*} M \rightarrow M
$$

is a unitary bimodule isomorphism. The group $\operatorname{Pin}^{c}(k)$ is a graded central extension of $O(k)$, graded by the sign of the determinant.

We now identify the twisting $\tau_{V}$ in the terms of 92.3 . Let $E \rightarrow X$ be the bundle of orthonormal frames in $V$. Thus $E \rightarrow X$ is a principal bundle with structure group $O(k)$. Write $P=E / / O(k), \tilde{P}=E / / \operatorname{Pin}^{\mathrm{c}}(k)$. Then

$$
P \rightarrow X
$$

is a local equivalence. and $\tilde{P} \rightarrow P$ is a graded central extension, defining a twisting $\tau$. Over $\tilde{P}$ we can form the bundle of bimodules

$$
\tilde{M}=(E \times M) / / \operatorname{Pin}^{\mathrm{c}}(k),
$$

giving a Morita equivalence between bundles of $\mathrm{C} \ell(V)$-modules and bundles of $C_{k^{-}}$ modules. In particular,

$$
H^{\prime}=\operatorname{hom}_{\mathrm{C} \ell(V)}(\tilde{M}, \mathrm{C} \ell(V) \otimes H)
$$

is a locally universal $\tau$-twisted $C_{k}$-module, and the map

$$
\begin{aligned}
\Gamma\left(\operatorname{Fred}_{\mathrm{C} \ell(V)}(\mathrm{C} \ell(V) \otimes H)\right) & \rightarrow \Gamma\left(\operatorname{Fred}^{(k)}\left(H^{\prime}\right)\right) \\
T & \mapsto T \circ(-)
\end{aligned}
$$


is a homeomorphism. Thus the group $K^{-\tau_{V}+n}(X)$ is isomorphic to the twisted $K$ group $K^{-\tau+n}(X)$, and, as in Donovan-Karoubi [14] we have a tautological Thom isomorphism

$$
K^{n}\left(X^{V}\right) \approx K^{-\tau+n}(X) .
$$

More generally, the same construction leads to a tautological Thom isomorphism

$$
K^{\sigma+n}(B(V), S(V)) \approx K^{-\tau+\sigma+n}(X),
$$

when $V$ is a vector bundle over a groupoid $X$.

With the Thom isomorphism in hand, one can define the pushforward, or umkehr map in the usual way. Let $f: X \rightarrow Y$ be a map of smooth manifolds, or a map of groupoids forming a bundle of smooth manifolds, $T=T_{X / Y}$ the corresponding relative (stable) tangent bundle, and $\tau_{0}$ the twisting on $X$ corresponding to $T$. Given a twisting $\tau$ on $Y$, and an isomorphism $f^{*} \tau \approx \tau_{0}$ one can combine the Pontryagin-Thom collapse with the Thom-isomorphism to form a pushforward map

$$
f_{!}: K^{f^{*} \sigma+n}(X) \rightarrow K^{-\tau+\sigma+n}(Y)
$$

where $\sigma$ is any twisting on $Y$. We leave the details to the reader.

We apply this to the situation in which $X=(G \times G) / / G, Y=G / / G$ (both with the adjoint action) and $X \rightarrow Y$ is the multiplication map $\mu$. In this case the twisting $\tau_{0}$ can be taken to be the twisting we denoted $\mathfrak{g}$ in the introduction. Since $\mathfrak{g}$ is pulled back from $\mathrm{pt} / / G$, there are canonical isomorphisms

$$
\mu^{*} \mathfrak{g} \approx p_{1}^{*} \mathfrak{g} \approx p_{2}^{*} \mathfrak{g} .
$$

We'll just write $\mathfrak{g}$ for any of these twistings. Suppose $\sigma$ is any twisting of $G / / G$ which is "primitive" in the sense that it comes equipped with an associative isomorphism $\mu^{*} \sigma \approx p_{1}^{*} \sigma+p_{2}^{*} \sigma$. Then the group $K_{G}^{\sigma+\mathfrak{g}}(G)$ acquires a Pontryagin product

$$
K_{G}^{\sigma+\mathfrak{g}}(G) \otimes K_{G}^{\sigma+\mathfrak{g}}(G) \rightarrow K_{G}^{\mu^{*} \sigma+2 \mathfrak{g}}(G \times G) \stackrel{\mu_{\mathfrak{l}}}{\longrightarrow} K_{G}^{\sigma+\mathfrak{g}}(G),
$$

making it into an algebra over $K_{G}^{0}(\mathrm{pt})=R(G)$.

3.7. The fundamental spectral sequence. Our basic technique of computation will be based on a variation of the Atiyah-Hirzebruch spectral sequence, which is constructed using the technique of Segal [30. The identification of the $E^{2}$-term depends only on the properties listed in 3.1 .

Suppose that $X$ is a local quotient groupoid, and write $\check{\mathcal{K}}^{\tau+t}$ for the presheaf on $[X]$ given by

$$
\check{\mathcal{K}}^{\tau+t}(U)=K^{\tau+t}\left(X_{U}\right) .
$$

Write

$$
\mathcal{K}^{\tau+t}=\operatorname{sh} \check{\mathcal{K}}^{\tau+t}
$$

for the associated sheaf. The limit of the Mayer-Vietoris spectral sequences associated to the (hyper-)covers of $[X]$ is a spectral sequence

$$
H^{s}\left([X] ; \mathcal{K}^{\tau+t}\right) \Longrightarrow K^{\tau+s+t}(X) .
$$

Since $X$ admits locally contractible slices the stalk of $\mathcal{K}^{\tau+t}$ at a point $c \in[X]$ is

$$
K^{\tau+t}\left(X_{c}\right) \approx \begin{cases}0 & t \text { odd } \\ \mathrm{R}^{\tau}\left(G_{x}\right) & t \text { even },\end{cases}
$$

where $x \in X_{0}$ is a representative of $c$, and $G_{x}=X(x, x)$. 
There is also a relative version. Suppose that $A \subset X$ is a pair of groupoids, and write $\mathcal{K}_{\text {rel }}^{\tau+t}$ for the sheaf on $[X]$ associated to the presheaf

$$
U \mapsto K^{\tau+t}\left(X_{U}, X_{[A] \cap U}\right)
$$

Then the limit of the Mayer-Vietoris spectral sequences associated to the hypercovers of $[X]$ gives

$$
H^{s}\left([X] ; \mathcal{K}_{\text {rel }}^{\tau+t}\right) \Longrightarrow K^{\tau+s+t}(X, A) .
$$

This spectral sequence is most useful when $A \subset X$ is closed, and has the property that for all sufficiently small $U \subset[X]$, the map $K^{\tau+t}\left(X_{U}\right) \rightarrow K^{\tau+t}\left(X_{[A] \cap U}\right)$ is surjective. In that case there is (for sufficiently small $U$ ) a short exact sequence

$$
K^{\tau+t}\left(X_{U}, X_{[A] \cap U}\right) \rightarrow K^{\tau+t}\left(X_{U}\right) \rightarrow K^{\tau+t}\left(X_{[A] \cap U}\right)
$$

and the sheaf $\mathcal{K}_{\text {rel }}^{\tau+t}$ can be identified with the extension of $i^{*} \mathcal{K}^{\tau+t}$ by zero

$$
\mathcal{K}_{\text {rel }}^{\tau+t}=i_{!}\left(\mathcal{K}^{\tau+t}\right),
$$

where $i: V \subset[X]$ is the inclusion of the complement of $A$. We will make use of this situation in the proof of Proposition 4.41,

\section{Computation of $K_{G}^{\tau}(G)$}

The aim of this section is to compute the groups $K_{G}^{\tau+*}(G)$ for non-degenerate $\tau$. We'll begin by considering general twistings, and adopt the non-degeneracy hypothesis as necessary. Our main results are Theorem 4.27, Corollary 4.38 and Corollary 4.39 .

4.1. Notation and assumptions. We first fix some notation. Let

- $G$ be a compact connected Lie group;

- $\mathfrak{g}$ the Lie algebra of $G$;

- $T$ a fixed maximal torus of $G$;

- $\mathfrak{t}$ the Lie algebra of $T$;

- $N$ the normalizer of $T$;

- $W=N / T$ the Weyl group;

- $\Pi=\operatorname{ker} \exp : \mathfrak{t} \rightarrow T$;

- $\Lambda=\operatorname{hom}(\Pi, Z)$, the character group of $T$;

- $N_{\text {aff }}^{e}=\Pi \rtimes N T$

- $W_{a f f}^{e}=\Pi \rtimes W=N_{a f f}^{e} / T$, the extended affine Weyl group;

The group $W_{a f f}^{e}$ can be identified the group of symmetries of $\mathfrak{t}$ generated by translations in $\Pi$ and the reflections in $W$. When $G$ is connected, the exponential map, from the orbit space $\mathfrak{t} / W_{a f f}^{e}$ to the space of conjugacy classes in $G$, is a homeomorphism.

We will make our computation for groups satisfying the equivalent conditions of the following lemma.

Lemma 4.1. For a Lie group $G$ the following are equivalent

i) For each $g \in G$ the centralizer $Z(g)$ is connected;

ii) $G$ is connected and $\pi_{1} G$ is torsion free;

iii) $G$ is connected and any central extension

$$
\mathbb{T} \rightarrow G^{\tau} \rightarrow G
$$

splits. 
Proof: The equivalence of (ii) and (iii) is elementary: Since $G$ is connected, its classifying space $B G$ is simply connected, and from the Hurewicz theorem and the universal coefficient theorem the torsion subgroup of $\pi_{1} G$ is isomorphic to the torsion subgroup of $H_{2}(B G)$, and so to the torsion subgroup of $H^{3}(B G ; \mathbb{Z})$. But for any compact Lie group the odd dimensional cohomology of the classifying space is torsion - the real cohomology of the classifying space is in even degrees (and is given by invariant polynomials on the Lie algebra).

The implication (ii) $\Longrightarrow$ (i) is [7, (3.5)]. For the converse (i) $\Longrightarrow$ (ii) we note first that $G=Z(e)$ is connected by hypothesis. Let $G^{\prime} \subset G$ denote the derived subgroup of $G$, the connected Lie subgroup generated by commutators in $G$, and $Z_{1} \subset G$ the connected component of the center of $G$. Set $A=Z\left(G^{\prime}\right) \cap Z_{1}$. Then from the principal fiber bundle $G^{\prime} \rightarrow G \rightarrow Z_{1} / A$ we deduce that the torsion subgroup of $\pi_{1} G$ is $\pi_{1} G^{\prime}$. We must show the latter vanishes. Now the inclusion $\pi_{1} Z(g) \rightarrow \pi_{1} G$ is surjective for any $g \in G$, since any centralizer contains a maximal torus $T$ of $G$ and the inclusion $\pi_{1} T \rightarrow \pi_{1} G$ is surjective - the flag manifold $G / T$ is simply connected. It follows that $Z(g)$ is connected if and only if the conjugacy class $G / Z(g)$ is simply connected. Furthermore, the conjugacy class in $G$ of an element of $G^{\prime}$ equals its conjugacy class in $G^{\prime}$, from which we deduce that all conjugacy classes in $G^{\prime}$ are connected and simply connected. Let $\widetilde{G^{\prime}}$ denote the simply connected (finite) cover of $G^{\prime}$. Then the set of conjugacy classes in $\widetilde{G^{\prime}}$ may be identified as a bounded convex polytope in the Lie algebra of a maximal torus, and furthermore $\pi_{1} G^{\prime}$ acts on it by affine transformations with quotient $\widetilde{G^{\prime}} / G^{\prime}$; see [16, §3.9]. The center of mass of the vertices of the polytope is a fixed point of the action. The finite group $\pi_{1} G^{\prime}$ acts freely on the corresponding conjugacy class of $\widetilde{G^{\prime}}$ with quotient a conjugacy class in $G^{\prime}$. Since the former is connected and the latter simply connected, it follows that $\pi_{1} G^{\prime}$ is trivial, as desired.

4.2. The main computation. Let $X=G / / G$ be the groupoid formed from $G$ acting on itself by conjugation. We will compute $K^{\tau+*}(X)=K_{G}^{\tau+*}(G)$ using the spectral sequence described in 93.7 . In this case it takes the form

$$
H^{s}\left(G / G ; \mathcal{K}^{\tau+t}\right) \Longrightarrow K_{G}^{\tau+s+t}(G) .
$$

The orbit space $G / G$ is the space of conjugacy classes in $G$, which is homeomorphic via the exponential map to $\mathfrak{t} / W_{\text {aff }}^{e}$. Our first task is to identify the sheaf $\mathcal{K}^{\tau+t}$ on $G / G \approx \mathfrak{t} / W_{\text {aff }}^{e}$.

Since $G / / G$ admits locally contractible slices, the stalk of $\mathcal{K}^{\tau+t}$ at a conjugacy class $c \in G / G$ is the twisted equivariant $K$-group

$$
K_{G}^{\tau+t}(c) .
$$

A choice of point $g \in c$ gives an identification $c=G / Z(g)$, and an isomorphism

$$
K_{G}^{\tau+t}(c) \approx K_{Z(g)}^{\tau_{g}+t}(\{g\}) \approx \begin{cases}\mathrm{R}^{\tau_{g}}(Z(g)) & t \text { even } \\ 0 & t \text { odd }\end{cases}
$$

We have denoted by $\tau_{g}$ the restriction of $\tau$ to $\{g\} / / Z(g)$, in order to emphasize the dependence on the choice of $g$. Among other things, this proves that

$$
\mathcal{K}^{\tau+\text { odd }}=0 .
$$


The twisting $\tau_{g}$ corresponds to a graded central extension

$$
\mathbb{T} \rightarrow \tilde{Z}(g) \rightarrow Z(g) .
$$

The group $Z(g)$ has $T$ for a maximal torus, and is connected when $G$ satisfies the equivalent conditions of Lemma 4.1. Denote

$$
\mathbb{T} \rightarrow \tilde{T} \rightarrow T
$$

the restriction of (4.4) to $T$. Then $\tilde{T}$ is a maximal torus in $\tilde{Z}(g)$. The map from the Weyl group of $\tilde{Z}(g)$ to the Weyl group $W_{g}$ of $Z(g)$ is an isomorphism, and

$$
\mathrm{R}^{\tau_{g}}(Z(g)) \rightarrow \mathrm{R}^{\tau_{g}}(T)^{W_{g}}
$$

is an isomorphism. We can therefore re-write (4.3) as

$$
K_{G}^{\tau+t}(c) \approx \begin{cases}\mathrm{R}^{\tau_{g}}(T)^{W_{g}} & t \text { even } \\ 0 & t \text { odd }\end{cases}
$$

We now reformulate these remarks in order to eliminate the explicit choice of $g \in c$. We can cut down the size of $c$ by requiring that $g$ lie in $T$. That helps, but it doesn't eliminate the dependence of $\tau_{g}$ on $g$. We can get rid of the reference to $g$ by choosing a geodesic in $T$ from each $g$ to the identity element, and using it to identify the twisting $\tau_{g}$ with $\tau_{0}$. This amounts to considering the set of elements of $\mathfrak{t}$ which exponentiate into $c$. This set admits a transitive action of $W_{a f f}^{e}$, and the stabilizer of an element $v$ is canonically isomorphic to $W_{g}$ where $g=\exp (v)$.

We are thus led to look at the groupoid $\mathfrak{t} / / T$, and the action of $W_{a f f}^{e}$. Writing it this way, however, does not conveniently display the action of $W_{a f f}^{e}$ on the twisting $\tau$. Following Example 2.32, we work instead with the weakly equivalent groupoid $\left(W_{\text {aff }}^{e} \times \mathfrak{t}\right) / / N_{a f f}^{e}$.

Consider the map

$$
K_{G}^{\tau+t}(G) \rightarrow K_{N_{a f f}^{e}}^{\tau+t}\left(W_{a f f}^{e} \times \mathfrak{t}\right)
$$

induced by

$$
\left(W_{\text {aff }}^{e} \times \mathfrak{t}\right) / / N_{\text {aff }}^{e} \stackrel{\text { projection }}{\longrightarrow} \mathfrak{t} / / N_{\text {aff }}^{e} \stackrel{\exp }{\longrightarrow} G / / G
$$

Since the right action of $W_{a f f}^{e}=N_{a f f}^{e} / T$ commutes with the diagonal left action of $N_{\text {aff }}^{e}$ on $W_{\text {aff }}^{e} \times \mathfrak{t}$, the group $W_{\text {aff }}^{e}$ acts on the groupoid $W_{\text {aff }}^{e} \times \mathfrak{t} / / N_{\text {aff }}^{e}$. The twisting $\tau$ is fixed by this action since it is pulled back from $G / / G$. The left action of $W_{\text {aff }}^{e}$ on $\left(W_{a f f}^{e} \times \mathfrak{t}\right) / / N_{a f f}^{e}$ therefore induces a right action of $W_{a f f}^{e}$ on $K_{N_{a f f}^{e}}^{\tau+t}(\mathfrak{t})$, and the image of $K_{G}^{\tau+t}(G)$ is invariant:

$$
K_{G}^{\tau+t}(G) \rightarrow K_{N_{a f f}^{e}}^{\tau+t}\left(W_{a f f}^{e} \times \mathfrak{t}\right)^{W_{a f f}^{e}} .
$$

Since $W_{a f f}^{e}=N_{a f f}^{e} / T$, the map

$$
\mathfrak{t} / / T \rightarrow\left(W_{\text {aff }}^{e} \times \mathfrak{t}\right) / / N_{\text {aff }}^{e}
$$

is a local equivalence, and so gives an isomorphism

$$
K_{N_{a f f}^{e}}^{\tau+t}\left(W_{a f f}^{e} \times \mathfrak{t}\right) \approx K_{T}^{\tau+t}(\mathfrak{t}) .
$$

There is therefore an action of $W_{a f f}^{e}$ on $K_{T}^{\tau+t}(\mathfrak{t})$, and we may re-write (4.7) as

$$
K_{G}^{\tau+t}(G) \rightarrow K_{T}^{\tau+t}(\mathfrak{t})^{W_{a f f}^{e}} .
$$


For $c \in G / G \approx \mathfrak{t} / W_{\text {aff }}^{e}$, let

$$
S_{c}=\{s \in \mathfrak{t} \mid \exp (s) \in c\}
$$

be the corresponding $W_{a f f}^{e}$-orbit in $\mathfrak{t}$. A similar discussion gives a map

$$
K_{G}^{\tau+t}(c) \rightarrow K_{N}^{\tau+t}\left(W_{a f f}^{e} \times S_{c}\right)^{W_{a f f}^{e}} \approx K_{T}^{\tau+t}\left(S_{c}\right)^{W_{a f f}^{e}} .
$$

Proposition 4.9. If $G$ satisfies the conditions of Lemma 4.1 then the map

$$
K_{G}^{\tau+t}(c) \rightarrow K_{T}^{\tau+t}\left(S_{c}\right)^{W_{a f f}^{e}}
$$

constructed above is an isomorphism.

Proof: Choose $v \in S_{c}$, and let $W_{v} \subset W_{a f f}^{e}$ be the stabilizer of $v$. We then have an identification $S_{c} \approx W_{\text {aff }}^{e} / W_{v}$, and so an isomorphism

$$
K_{T}^{\tau+t}\left(S_{c}\right)^{W_{a f f}^{e}} \approx K_{T}^{\tau+t}(\{v\})^{W_{v}} .
$$

Write $g=\exp (v)$. The restriction of $N_{a f f}^{e} \rightarrow G$ identifies $W_{v}$ with the Weyl group of $Z(g),\{v\} / / T$ with $\{g\} / / T$, and the restriction of $\tau$ to $\{v\} / / T$ with $\tau_{g}$. By Example 2.33 action of $W_{v}$ on $K_{T}^{\tau+t}(\{v\})$ coincides with the action $W_{v}$ by conjugation. The result then follows from (4.6).

We now identify the sheaf $\mathcal{K}^{\tau+t}$. Since $\{0\} \rightarrow \mathfrak{t}$ is an equivariant homotopy equivalence, the restriction map

$$
K_{T}^{\tau+t}\left(S_{c} \times \mathfrak{t}\right) \rightarrow K_{T}^{\tau+t}\left(S_{c} \times\{0\}\right)
$$

is an isomorphism. Next note that the aggregate of the restriction maps to the points of $S_{c}$ gives a map from

$$
K_{T}^{\tau+t}\left(S_{c} \times \mathfrak{t}\right)^{W_{a f f}^{e}}
$$

to the set of $W_{a f f}^{e}$-equivariant maps

$$
S_{c} \rightarrow K_{T}^{\tau+t}(\mathfrak{t}),
$$

which, using the fact that $W_{a f f}^{e}$ acts transitively on $S_{c}$, is easily checked to be an isomorphism. Write $p: \mathfrak{t} \rightarrow \mathfrak{t} / W_{a f f}^{e}$ for the projection, and for an open $U \subset G / G=$ $\mathfrak{t} / W_{\text {aff }}^{e}$ set

$$
S_{U}=p^{-1}(U) .
$$

Let $\mathcal{F}^{\tau+t}$ be the presheaf which associates to $U \subset G / G$ the set of locally constant $W_{a f f}^{e}$-equivariant maps

$$
S_{U} \rightarrow K_{T}^{\tau+t}(\mathfrak{t}) .
$$

There is then a map of presheaves

$$
\check{\mathcal{K}}^{\tau+t} \rightarrow \mathcal{F}^{\tau+t},
$$

hence a map of sheaves

$$
\mathcal{K}^{\tau+t} \rightarrow \mathcal{F}^{\tau+t}
$$

Corollary 4.11. The map (4.10) is an isomorphism. 
Proof: Proposition 4.9 implies that (4.10) is an isomorphism of stalks, hence an isomorphism.

We now re-interpret the sheaf $\mathcal{F}$ in a form more suitable to describing its cohomology. Since

$$
\mathfrak{T w i s t}_{\mathfrak{t} / / T} \rightarrow \mathfrak{T w i s t}_{\{0\} / / T}
$$

is an equivalence of categories, the restriction of $\tau$ to $\mathfrak{t} / / T$ corresponds to a graded central extension

$$
\mathbb{T} \rightarrow T^{\tau} \rightarrow T
$$

equipped with an action of $W_{a f f}^{e}$. The $W_{a f f}^{e}$-action fixes $\mathbb{T}$ and acts on $T$ through its quotient $W$, the Weyl group. Write $\Lambda^{\tau}$ for the set of splittings of (4.12). Note that $\Lambda^{\tau}$ is a torsor for $\Lambda$ and inherits a compatible $W_{a f f}^{e}$ action from (4.12).

By Proposition 3.5 the group

$$
K_{T}^{\tau+0}(\mathfrak{t}) \approx K_{T}^{\tau+0}(\{0\})
$$

may be identified with with the set of compactly supported functions on $\Lambda^{\tau}$ with values in $\mathbb{Z}$. We will see shortly that the action of $W_{\text {aff }}^{e}$ is the combination of its natural action on $\Lambda^{\tau}$ and an action on $\mathbb{Z}$ given by a homomorphism $\epsilon: W_{\text {aff }}^{e} \rightarrow$ $\mathbb{Z} / 2$. Writing $\mathbb{Z}(\epsilon)$ for the sign representation associated to $\epsilon$, we then have an isomorphism of $W_{\text {aff }}^{e}$-modules

$$
K_{T}^{\tau+0}(\{0\}) \approx \operatorname{Hom}_{c}\left(\Lambda^{\tau}, \mathbb{Z}(\epsilon)\right) .
$$

To verify the claim about the action first note that the automorphism group of the restriction of $\tau$ to $\mathfrak{t} / / T$ is

$$
H^{2}(B T ; \mathbb{Z}) \times H^{0}(B T ; \mathbb{Z} / 2) \approx \Lambda \times \mathbb{Z} / 2 \approx R(T)^{\times} \approx K_{T}^{0}(\mathrm{pt})^{\times}
$$

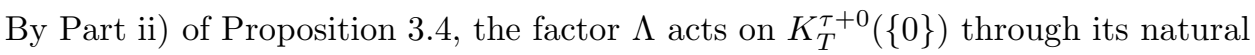
action on $\Lambda^{\tau}$, while the $\mathbb{Z} / 2$ acts by its sign representation.

Since $W_{a f f}^{e}=\Pi \rtimes W$, and the action of $W_{a f f}^{e}$ on $K_{T}^{\tau+0}(\{0\})$ is determined by its restriction to $\Pi$ and $W$. The group $\Pi$ acts trivially on $T$ and so it acts on $K_{T}^{\tau+0}(\{0\})$ through a homomorphism

$$
\Pi \stackrel{\left(b, \epsilon_{\Pi}\right)}{\longrightarrow} \Lambda \times \mathbb{Z} / 2 .
$$

The group $W$ does act on $T$, and so on

$$
H^{2}(B T ; \mathbb{Z}) \times H^{0}(B T ; \mathbb{Z} / 2) \approx \Lambda \times \mathbb{Z} / 2,
$$

by the product of the natural (reflection) action on $\Lambda$ and the trivial action on $\mathbb{Z} / 2$. The restriction of the action of $W_{a f f}^{e}$ to $W$ is therefore determined by a crossed homomorphism

$$
W \rightarrow \Lambda
$$

compatible with $b$, and an ordinary homomorphism $\epsilon_{W}: W \rightarrow \mathbb{Z} / 2$. The maps $\epsilon_{\Pi}$ and $\epsilon_{W}$ combine to give the desired map $\epsilon: W_{\text {aff }}^{e} \rightarrow \mathbb{Z} / 2$, while the map $b: \Pi \rightarrow \Lambda$ and the crossed homomorphism $W \rightarrow \Lambda$ correspond to the natural action of $W_{a f f}^{e}$ on $\Lambda^{\tau}$. This verifies the isomorphism (4.13) of $W_{a f f}^{e}$-modules.

We can now give a useful description of $\mathcal{F}$. First recall a construction. Suppose $X$ is a space equipped with an action of a group $\Gamma$, and that $\mathcal{G}$ is an equivariant sheaf on $X$. Write $p: X \rightarrow X / \Gamma$ for the projection to the orbit space. There is then a sheaf, $\left(p_{*} \mathcal{G}\right)^{\Gamma}$ on $X / \Gamma$ whose value on an open set $V$ is the set of $\Gamma$-invariant elements of $\mathcal{G}\left(p^{-1} V\right)$. A very simple situation is when $\mathcal{G}$ is the constant sheaf $\mathbb{Z}$. 
In that case $\left(p_{*} \mathcal{G}\right)^{\Gamma}$ is again the constant sheaf $\mathbb{Z}$. This will be useful in the proof of Proposition 4.18 below.

Corollary 4.14. Write

$$
\tilde{\mathfrak{t}}=\mathfrak{t} \times W_{a f f}^{e} \Lambda^{\tau}
$$

and let

$$
\begin{aligned}
& p: \mathfrak{t} \times \Lambda^{\tau} \rightarrow \tilde{\mathfrak{t}} \text { and } \\
& f: \tilde{\mathfrak{t}} \rightarrow \mathfrak{t} / W_{\text {aff }}^{e}
\end{aligned}
$$

denote the projections. There is a canonical isomorphism

$$
\mathcal{F}^{\tau+0} \approx f_{*}^{c}\left(p_{*} \mathbb{Z}(\epsilon)^{W_{a f f}^{e}}\right),
$$

where $f_{*}^{c}$ denotes pushforward with proper supports.

To go further we need to make an assumption.

Assumption 4.15. The twisting $\tau$ is non-degenerate in the sense that $b$ is a monomorphism.

In terms of the classification of twistings, this is equivalent to requiring that the image of the isomorphism class of $\tau$ in

$$
H_{T}^{3}(T ; \mathbb{R}) \approx H^{1}(T ; \mathbb{R}) \otimes H^{1}(T ; \mathbb{R})
$$

is a non-degenerate bilinear form.

Next note

Lemma 4.16. The map $\epsilon_{W}: W \rightarrow \mathbb{Z} / 2$ is trivial.

Proof: The homomorphism in question corresponds to the element in $H_{W}^{1}(\mathrm{pt})=$ $H^{1}(B W ; \mathbb{Z} / 2)$ given by restricting the isomorphism class of the twisting along

$$
\begin{aligned}
H_{G}^{1}(G ; \mathbb{Z} / 2) \times H_{G}^{3}(G ; \mathbb{Z}) \rightarrow H_{G}^{1}(G ; \mathbb{Z} / 2) \rightarrow & H_{G}^{1}(\{e\} ; \mathbb{Z} / 2) \\
& \rightarrow H_{N}^{1}(\{e\} ; \mathbb{Z} / 2) \approx H_{W}^{1}(\{e\} ; \mathbb{Z} / 2) .
\end{aligned}
$$

Since $G$ is assumed to be connected $H_{G}^{1}(\mathrm{pt})=0$ and the result follows.

Corollary 4.17. There is an isomorphism $\mathbb{Z} \approx \mathbb{Z}(\epsilon)$ of equivariant sheaves on $\mathfrak{t} \times \Lambda^{\tau}$.

Proof: The sheaf $\mathbb{Z}(\epsilon)$ is classified by the element

$$
\tilde{\epsilon} \in H_{W_{a f f}^{e}}^{1}\left(\mathfrak{t} \times \Lambda^{\tau} ; \mathbb{Z} / 2\right)
$$

pulled back from the $\epsilon \in H_{W_{a f f}^{e}}^{1}(\mathrm{pt} ; \mathbb{Z} / 2)$. By Corollary 4.16 the restriction of $\tilde{\epsilon}$ to $W$ is trivial. By Assumption 4.15 , the group $\Pi$ acts freely on $\Lambda^{\tau}$, so the restriction of $\tilde{\epsilon}$ to $H_{W_{a f f}^{e}}^{1}\left(\mathfrak{t} \times \Lambda^{\tau} ; \mathbb{Z} / 2\right)$ is also trivial. This proves that $\tilde{\epsilon}=0$.

Proposition 4.18. There is an isomorphism

$$
\mathcal{F}^{\tau+0} \approx f_{*}^{c}(\mathbb{Z}),
$$

where $f: \tilde{\mathfrak{t}}=\mathfrak{t} \times{ }_{W_{a f f}^{e}} \Lambda^{\tau} \rightarrow \mathfrak{t} / W$ is the projection and $f_{*}^{c}$ denotes pushforward with proper supports. 
Proof: By Corollaries 4.17 and 4.14 there is an isomorphism

$$
\mathcal{F}^{\tau+0} \approx f_{*}^{c}\left(p_{*} \mathbb{Z}^{W_{a f f}^{e}}\right),
$$

so the result follows from the fact that $p_{*} \mathbb{Z}^{W_{a f f}^{e}} \approx \mathbb{Z}$.

Finally, using the existence of contractible local slices we can describe the cohomology of $\mathcal{K}^{\tau+0} \approx \mathcal{F}^{\tau+0}$.

Lemma 4.19. The edge homomorphism of the Leray spectral sequence for $f$ is an isomorphism

$$
H^{*}\left(\mathfrak{t} / W_{a f f}^{e} ; \mathcal{K}^{\tau}\right) \approx H_{c}^{*}(\tilde{\mathfrak{t}} ; \mathbb{Z})
$$

where $H_{c}^{*}$ denotes cohomology with compact supports.

To calculate $H_{c}^{*}(\tilde{\mathfrak{t}} ; \mathbb{Z})$ we need to understand the structure of $\tilde{\mathfrak{t}}$. This amounts to describing more carefully the action of $W_{a f f}^{e}$ on $\Lambda^{\tau}$.

Lemma 4.20. There exists an element $\lambda_{0} \in \Lambda^{\tau}$ fixed by $W$.

Proof: The inclusion $\{0\} / / T \subset \mathfrak{t} / / T$ is equivariant for the action of $W$. We can therefore study the action of $W$ on the central extension of $T$ defined by the restriction of $\tau$ to $\{0\} / / T$. Now $\tau$ started out as a twisting of $G / / G$, so our twisting of $\{0\} / / T$ is the restriction of a twisting $\tau_{G}$ of $\{e\} / / G$. Moreover, the action of $W$ is derived from the action of inner automorphisms of $G$ on $\tau_{G}$. Now the twisting $\tau_{G}$ corresponds to a central extension

$$
\mathbb{T} \rightarrow G^{\tau} \rightarrow G .
$$

By our assumptions on $G$ (Lemma 4.1), this central extension splits. Choose a splitting

$$
G^{\tau} \rightarrow \mathbb{T}
$$

and let $\lambda_{0} \in \Lambda^{\tau}$ be the composition

$$
\tilde{T} \rightarrow G^{\tau} \rightarrow \mathbb{T} .
$$

Since $\mathbb{T}$ is abelian, the splitting (4.21) is preserved by inner automorphisms of $G$. It follows that splitting $e$ is fixed by the inner automorphisms of $\tilde{G}$ which normalize $\tilde{T}$. The claim follows.

Remark 4.22. Any two choices of $\lambda_{0}$ differ by a character of $G$, so the element $\lambda_{0}$ is unique if an only if the character group of $G$ is trivial. Since we've assumed that $G$ is connected and $\pi_{1} G$ is torsion free, this is in turn equivalent to requiring that $G$ be simply connected.

Remark 4.23. A primitive twisting $\tau$ comes equipped with a trivialization of its restriction to $\{e\} / / G$, or in other words a splitting of the graded central extension $G^{\tau} \rightarrow G$. A primitive twisting therefore comes equipped with a canonical choice of $\lambda_{0}$.

Using a fixed choice of $\lambda_{0}$, we can identify $\Lambda^{\tau}$ with $\Lambda$ as a $W$-space. To sum up, we can make an identification $\Lambda^{\tau} \approx \Lambda$, the action of $\Pi$ is given by a $W$-equivariant homomorphism $\Pi \rightarrow \Lambda$, and the $W$-action is the natural one on $\Lambda$. 
Lemma 4.24. When $\tau$ is non-degenerate the $W_{\text {aff }}^{e}$-set $\Lambda^{\tau}$ admits an (equivariant) embedding in $\mathbf{t}$. There are finitely many $W_{\text {aff }}^{e}$-orbit in $\Lambda^{\tau}$, and each orbits is of the form $W_{\text {aff }}^{e} / W_{c}$, with $\left(W_{c}, \mathfrak{t}\right)$ a finite (affine) reflection group.

Proof: Since $b$ is a monomorphism the map $t=\Pi \otimes \mathbb{R} \rightarrow \Lambda \otimes \mathbb{R}$ is an isomorphism. The first assertion now follows from our identification of $\Lambda^{\tau}$ with $\Lambda$. As for the finiteness of the number of orbits, since $b$ is a monomorphism, the group $\Lambda / \Pi$ is finite, and there are already only finitely many $\Pi$-orbits in $\Lambda^{\tau}$. The remaining assertions follow from standard facts about the action of $W_{\text {aff }}^{e}$ on $\mathfrak{t}$ (Propositions 4.46 and 4.47 below).

Corollary 4.25. When $\tau$ is non-degenerate, there is a homeomorphism

$$
\tilde{\mathfrak{t}} \equiv \coprod_{s \in S} \mathfrak{t} / W_{s}
$$

with $S$ finite, and $W_{s}$ a finite reflection group of isometries of $\mathfrak{t}$. Moreover

$$
\mathfrak{t} / W_{s} \equiv \mathbb{R}^{n_{1}} \times[0, \infty)^{n_{2}}
$$

with $n_{2}=0$ if and only if $W_{s}$ is trivial.

Proof: This is immediate from Lemma 4.24 above and Proposition 4.47 below.

Since $H_{c}^{*}([0, \infty) ; \mathbb{Z})=0$ and

$$
H_{c}^{*}(\mathbb{R} ; \mathbb{Z})= \begin{cases}\mathbb{Z} & *=1 \\ 0 & \text { otherwise }\end{cases}
$$

the Kunneth formula gives

$$
H_{c}^{*}\left(\mathbb{R}^{n_{1}} \times[0, \infty)^{n_{2}} ; \mathbb{Z}\right)= \begin{cases}\mathbb{Z} & n_{2}=0 \text { and } *=n_{1} \\ 0 & \text { otherwise. }\end{cases}
$$

In summary, we have

Proposition 4.26. The cohomology group $H_{c}^{*}(\tilde{\mathfrak{t}} ; \mathbb{Z})$ is zero unless $*=n$, and $H_{c}^{n}(\tilde{\mathfrak{t}} ; \mathbb{Z})$ is isomorphic to the free abelian group on the set of free $W_{\text {aff }}^{e}$-orbits in $\Lambda^{\tau}$. More functorially,

$$
H_{c}^{n}(\tilde{\mathfrak{t}} ; \mathbb{Z}) \approx \operatorname{Hom}_{W_{a f f}^{e}}\left(\Lambda^{\tau}, H_{c}^{n}(\mathfrak{t}) \otimes \mathbb{Z}(\epsilon)\right) .
$$

Proposition 4.26 implies that the spectral sequence (4.2) collapses, giving

Theorem 4.27. Suppose that $G$ is a Lie group of rank $n$ satisfying the conditions of Lemma 4.1. and that $\tau$ is a non-degenerate twisting of $G / / G$, classified by

$$
[\tau] \in H_{G}^{3}(G ; \mathbb{Z}) \times H_{G}^{1}(G ; \mathbb{Z} / 2) .
$$

The restriction of $\tau$ to $\mathrm{pt} / / \mathrm{T}$ determines a central extension

$$
\mathbb{T} \rightarrow T^{\tau} \rightarrow T
$$


with an action of $W_{a f f}^{e}$. Write $\Lambda^{\tau}$ for the set of splittings of (4.28), $\epsilon: W_{a f f}^{e} \rightarrow \mathbb{Z} / 2$ for the map corresponding to the restriction of $[\tau]$ to

$$
H_{N}^{1}(T ; \mathbb{Z} / 2) \approx H^{1}\left(W_{a f f}^{e} ; \mathbb{Z} / 2\right),
$$

and $\mathbb{Z}(\epsilon)$ for the associated sign representation. Then $K_{G}^{\tau+n+1}(G)=0$, and the twisted $K$-group $K_{G}^{\tau+n}(G)$ is given by

$$
K_{G}^{\tau+n}(G) \approx \operatorname{Hom}_{W_{a f f}^{e}}\left(\Lambda^{\tau}, H_{c}^{n}(\mathfrak{t}) \otimes \mathbb{Z}(\epsilon)\right),
$$

which can be identified with the free abelian group on the set of free $W_{a f f}^{e}$-orbits in $\Lambda^{\tau}$, after choosing a point in each free orbit. This isomorphism is natural in the sense that if $i: H \subset G$ is a subgroup of rank $n$ also satisfying the conditions of Lemma 4.1, then the restriction map

$$
K_{G}^{\tau+n}(G) \rightarrow K_{H}^{\tau+n}(H)
$$

is given by the inclusion

$$
\operatorname{Hom}_{W_{a f f}^{e}(G)}\left(\Lambda^{\tau}, H_{c}^{n}(\mathfrak{t}) \otimes \mathbb{Z}(\epsilon)\right) \subset \operatorname{Hom}_{W_{a f f}^{e}(H)}\left(\Lambda^{\tau}, H_{c}^{n}(\mathfrak{t}) \otimes \mathbb{Z}(\epsilon)\right) .
$$

Remark 4.29. In Theorem 4.27 the group $W_{a f f}^{e}$ acts on $H_{c}^{n}(\mathfrak{t})$ through the action of $W$ on $\mathfrak{t}$. The reflections thus act by $(-1)$ and a choice of orientation on $\mathfrak{t}$ identifies $H_{c}^{n}(\mathfrak{t})$ with the usual sign representation of $W$ on $\mathbb{Z}$.

The group $K_{G}^{\tau+n}(G)$ is a module over $R(G)$. Our next goal is to identify this module structure. Because $G$ is connected we can identify $R(G)$ with the ring of $W$ invariant elements of $\mathbb{Z}[\Lambda]$ or with the convolution algebra of compactly supported functions $\operatorname{Hom}_{c}(\Lambda, \mathbb{Z})$. The algebra $\operatorname{Hom}_{c}(\Lambda, \mathbb{Z})$ acts on $\operatorname{Hom}\left(\Lambda^{\tau}, H_{c}^{n}(\mathfrak{t}) \otimes \mathbb{Z}(\epsilon)\right)$ by convolution, and one easily checks that the $W$-invariant elements preserve the $W_{a f f}^{e}$-equivariant functions.

Proposition 4.30. Under the identification

$$
K_{G}^{\tau+n}(G) \approx \operatorname{Hom}_{W_{a f f}^{e}}\left(\Lambda^{\tau}, H_{c}^{n}(\mathfrak{t}) \otimes \mathbb{Z}(\epsilon)\right)
$$

the action of $R(G) \approx \operatorname{Hom}_{c}(\Lambda, \mathbb{Z})^{W}$ corresponds to convolution of functions.

Proof: This is straightforward to check in case $G$ is a torus. The case of general $G$ is reduced to this case by looking at the restriction map to a maximal torus and using Theorem 4.27 .

Proposition 4.30 leads to a very useful description of $K_{G}^{\tau+n}(G)$. Choose an orientation of $\mathfrak{t}$ and hence an identification $H_{c}^{n}(\mathfrak{t}) \approx \mathbb{Z}$ of abelian groups. By definition, the elements of $\Lambda^{\tau}$ are characters of $T^{\tau}$, all of which restrict to the defining character of $\mathbb{T}$. To a function

$$
f \in \operatorname{Hom}_{\Pi}\left(\Lambda^{\tau}, H_{c}^{n}(\mathfrak{t}) \otimes \mathbb{Z}(\epsilon)\right) \approx \operatorname{Hom}_{\Pi}\left(\Lambda^{\tau}, \mathbb{Z}(\epsilon)\right)
$$

we associate the series

$$
\delta_{f}=\sum_{\lambda \in \Lambda^{\tau}} f(\lambda) \lambda^{-1}
$$


which is the Fourier expansion of the distribution on $T^{\tau}$ satisfying $\delta_{f}(\lambda)=f(\lambda)$. Our next aim is to work out more explicitly which distribution it is, especially when $f$ comes from an element of

$$
\operatorname{Hom}_{W_{a f f}^{e}}\left(\Lambda^{\tau}, H_{c}^{n}(\mathfrak{t}) \otimes \mathbb{Z}(\epsilon)\right) \approx \operatorname{Hom}_{W_{a f f}^{e}}\left(\Lambda^{\tau}, \mathbb{Z}(\epsilon)\right) .
$$

Since all of the characters $\lambda$ restrict to the defining character of the central $\mathbb{T}$, we'll think of the distribution $\delta_{f}$ as acting on the space of functions $g: T^{\tau} \rightarrow \mathbb{C}$ satisfying $g(\zeta v)=\zeta g(v)$ for $\zeta \in \mathbb{T}$. This space is the space of sections of a suitable complex line bundle $L_{\tau}$ over $T$. The character $\chi$ of a representation of $G$ is a function on $T$, and action of $\chi$ on $\delta_{f}$ is given by

$$
\chi \cdot \delta_{f}(g)=\delta_{f}(g \cdot \chi) .
$$

Since $\Pi$ and $\Lambda$ are duals, we have $\operatorname{hom}(\Pi, \mathbb{Z} / 2)=\Lambda \otimes \mathbb{Z} / 2$, and we may regard $\epsilon_{\Pi}$ as an element of $\Lambda / 2 \Lambda$. This determines an element

$$
\lambda_{\epsilon}=\frac{1}{2} \epsilon_{\Pi} \in \frac{1}{2} \Lambda / \Lambda \subset \Lambda \otimes \mathbb{R} / \mathbb{Z} .
$$

Thinking of $\Pi$ as the character group of $\Lambda \otimes \mathbb{R} / \mathbb{Z}$, the function $\epsilon_{\Pi}: \Pi \rightarrow \mathbb{Z} / 2$ is given by evaluation of characters on $\lambda_{\epsilon}$ :

$$
\epsilon_{\Pi}(\pi)=\pi\left(\lambda_{\epsilon}\right) .
$$

Since $\epsilon_{\Pi}$ is $W$-invariant, so is $\lambda_{\epsilon}$.

From the embedding $b: \Pi \subset \Lambda$ we get a map

$$
b: T=\Pi \otimes \mathbb{R} / \mathbb{Z} \rightarrow \Lambda \otimes \mathbb{R} / \mathbb{Z} .
$$

We'll write $F=\Lambda / \Pi$ for the kernel of this map, and $F_{\epsilon}$ for the inverse image of $\lambda_{\epsilon}$. Set

$$
F^{\tau}=\Lambda^{\tau} / \Pi .
$$

The elements of $F^{\tau}$ can be interpreted as sections of the restriction of $L_{\tau}$ to $F$. Finally, let $F_{\epsilon \text {,reg }} \subset F_{\epsilon}$ and $F_{\text {reg }}^{\tau} \subset F^{\tau}$ be the subsets consisting of elements on which the Weyl group $W$ acts freely.

Proposition 4.32. For

$$
f \in \operatorname{Hom}_{\Pi}\left(\Lambda^{\tau}, H_{c}^{n}(\mathfrak{t}) \otimes \mathbb{Z}(\epsilon)\right) \approx \operatorname{Hom}_{\Pi}\left(\Lambda^{\tau}, \mathbb{Z}(\epsilon)\right)
$$

The value of the distribution $\delta_{f}$ on a section $g$ of $L_{\tau}$ is given by

$$
\delta_{f}(g)=\frac{1}{|F|} \sum_{(\lambda, x) \in F^{\tau} \times F_{\epsilon}} f(\lambda) \lambda^{-1}(x) g(x)
$$

When $f$ is $W_{\text {aff }}^{e}$-invariant, then

$$
\delta_{f}(g)=\frac{1}{|F|} \sum_{(\lambda, x) \in F_{\text {reg }}^{\tau} \times F_{\epsilon, r e g}} f(\lambda) \lambda^{-1}(x) g(x)
$$

Proof: Let's first check that (4.33) is well-defined. Under

$$
\lambda \mapsto \lambda \pi \quad \pi \in \Pi,
$$


the term $f(\lambda) \lambda^{-1}(x) g(x)$ gets sent to

$$
\begin{aligned}
f(\lambda \pi)(\lambda \pi)^{-1}(x) g(x) & =\epsilon(\pi) f(\lambda) \lambda^{-1}(x) \pi^{-1}(x) g(x) \\
& =\epsilon(\pi) f(\lambda) \lambda^{-1}(x) \epsilon\left(\pi^{-1}\right) g(x) \\
& =f(\lambda) \lambda^{-1}(x) g(x)
\end{aligned}
$$

so $f(\lambda) \lambda^{-1}(x) g(x)$ does indeed depend only on the $\Pi$-coset of $\lambda$.

To establish (4.33), it suffices by linearity to consider the case in which $\delta_{f}$ is of the form

$$
\delta_{f}=\lambda^{-1} \cdot \delta_{\Pi}
$$

with $\lambda \in \Lambda^{\tau}$,

$$
\delta_{\Pi}=\sum_{\pi \in \Pi} \epsilon(\pi) \pi^{-1}
$$

and $g$ is an element of $\Lambda^{\tau}$. In this case $f$ vanishes off of the $\Pi$-orbit through $\lambda$, and $f(\lambda)=1$. The sum (4.33) is then

$$
\frac{1}{|F|} \sum_{x \in F_{\epsilon}} \lambda^{-1}(x) g(x)
$$

By definition, for $\eta \in \Pi$,

$$
\delta_{\Pi}(\eta)=\eta\left(\lambda_{\epsilon}\right)=\frac{1}{|F|} \sum_{x \in F_{\epsilon}} \eta(x)
$$

For $\eta \in \Lambda \backslash \Pi$, there is an $a \in F$ with $\eta(a) \neq 1$. In that case

$$
\frac{1}{|F|} \sum_{x \in F_{\epsilon}} \eta(x)=\frac{1}{|F|} \sum_{x \in F_{\epsilon}} \eta(a x)=\eta(a) \frac{1}{|F|} \sum_{x \in F_{\epsilon}} \eta(x),
$$

SO

$$
\frac{1}{|F|} \sum_{x \in F_{\epsilon}} \eta(x)=0 .
$$

It follows that for every $\eta \in \Lambda$,

$$
\delta_{\Pi}(\eta)=\frac{1}{|F|} \sum_{x \in F_{\epsilon}} \eta(x)
$$

Now suppose $g \in \Lambda^{\tau}$. Then

$$
\delta_{f}(g)=\delta_{\Pi}\left(\lambda^{-1} g\right)=\frac{1}{|F|} \sum_{x \in F_{\epsilon}} \lambda^{-1}(x) g(x),
$$

which is (4.34). This proves the first assertion of Proposition 4.32

For the second assertion, note that if $\lambda \in F_{\text {reg }}^{\tau}$ is fixed by an element of $W$ it is fixed by an element $w \in W$ which is a reflection. By Weyl-equivariance, we have $f(\lambda)=f(w \lambda)=w \cdot f(\lambda)=-f(\lambda)$, and so $f(\lambda)=0$. This gives

$$
\delta_{f}(g)=\frac{1}{|F|} \sum_{(\lambda, x) \in F_{\text {reg }}^{\tau} \times F} f(\lambda) \lambda^{-1}(x) g(x) .
$$


If $x \in F$ is an element fixed by a reflection $w \in W$ then

$$
\begin{aligned}
\sum_{\lambda \in F_{\text {reg }}^{\tau}} f(\lambda) \lambda^{-1}(x) g(x) & =\sum_{\lambda \in F_{\text {reg }}^{\tau}} f(\lambda) \lambda^{-1}(w \cdot x) g(x) \\
& =\sum_{\lambda \in F_{\text {reg }}^{\tau}} f(\lambda)\left(\lambda^{w}\right)^{-1}(x) g(x) \\
& =\sum_{\lambda \in F_{\text {reg }}^{\tau}} f\left(\lambda^{w}\right) \lambda^{-1}(x) g(x) \\
& =-\sum_{\lambda \in F_{\text {reg }}^{\tau}} f(\lambda) \lambda^{-1}(x) g(x)
\end{aligned}
$$

so the terms involving such an $x$ sum to zero, and

$$
\delta_{f}(g)=\frac{1}{|F|} \sum_{(\lambda, x) \in F_{\mathrm{reg}}^{\tau} \times F_{\epsilon, \mathrm{reg}}} f(\lambda) \lambda^{-1}(x) g(x)
$$

Let $I^{\tau} \subset R(G)$ be the ideal consisting of virtual representations whose character vanishes on the elements of $F_{\epsilon, \text { reg. }}$.

Corollary 4.35. The ideal $I^{\tau}$ annihilates $K_{G}^{\tau+*}(G)$.

Proof: Write $\chi$ for the character of an element of $I^{\tau}$. For $f \in \operatorname{Hom}_{W_{a f f}^{e}}\left(\Lambda^{\tau}, H_{c}^{n}(\mathfrak{t}) \otimes\right.$ $\mathbb{Z}(\epsilon))$ we have, by Proposition 4.32

$$
\chi \delta_{f}(g)=\delta_{f}(g \cdot \chi)=\sum_{(\lambda, x) \in F_{\mathrm{reg}}^{\tau} \times F_{\mathrm{reg}}} f(\lambda) \lambda^{-1}(x) g(x) \chi(x)=0 .
$$

Remark 4.36. The conjugacy classes in $G$ of the elements in $F_{\epsilon, \text { reg }}$ are known as the Verlinde conjugacy classes, and the ideal $I^{\tau}$ as the Verlinde ideal.

Proposition 4.37. The $R(G)$-module $K_{G}^{\tau+n}(G)$ is cyclic.

Proof: Using Lemma 4.20 choose a $W_{a f f}^{e}$-equivariant isomorphism $\Lambda^{\tau} \approx \Lambda$, and an orientation of $\mathfrak{t}$ giving an isomorphism $H_{c}^{n}(\mathfrak{t}) \approx \mathbb{Z}$. We can then identify $K_{G}^{\tau+n}(G)$ with

$$
\operatorname{Hom}_{W_{a f f}^{e}}(\Lambda, \mathbb{Z}(\epsilon)),
$$

though we remind the reader that $W_{\text {aff }}^{e}$ acts on $\mathbb{Z}$ through its sign representation. We'll continue the convention of writing elements

$$
f \in \operatorname{Hom}_{W_{a f f}^{e}}(\Lambda, \mathbb{Z})
$$

as Fourier series

$$
\sum f(\lambda) \lambda^{-1} .
$$

Set

$$
\delta_{\Pi}=\sum_{\pi \in \Pi} \epsilon(\pi) \pi^{-1},
$$


and for $\lambda \in \Lambda$ write

$$
a(\lambda)=\sum_{w \in W}(-1)^{w} w \cdot \lambda
$$

Then the elements

$$
a(\lambda) * \delta_{\Pi}
$$

span $\operatorname{Hom}_{W_{a f f}^{e}}(\Lambda, \mathbb{Z}(\epsilon))$. Since $\pi_{1} G$ is torsion-free, there is an exact sequence

$$
G^{\prime} \rightarrow G \rightarrow J
$$

where $J$ is a torus, and $G^{\prime}$ is simply connected. The character group of $J$ is the subgroup $\Lambda^{W}$ of Weyl-invariant elements of $\Lambda$, and the weight lattice for $G^{\prime}$ is the quotient $\Lambda / \Lambda^{W}$. Choose a Weyl chamber for $G$ and let $\rho \in \Lambda \otimes \mathbb{Q}$ be $1 / 2$ the sum of the positive roots of $G$ (which we will write as a product of square roots of elements in our Fourier series notation). Since $J$ is a torus, the image $\rho^{\prime}$ of $\rho$ in $\Lambda / \Lambda^{W} \otimes \mathbb{Q}$ is $1 / 2$ the sum of the positive roots of $G^{\prime}$, which since $G^{\prime}$ is simply connected, lies in $\Lambda / \Lambda^{W}$. Let $\tilde{\rho} \in \Lambda$ be any element congruent to $\rho^{\prime}$ modulo $\Lambda^{W}$. Claim: for any $\lambda \in \Lambda$, the ratio

$$
\frac{a(\lambda)}{a(\tilde{\rho})}
$$

is the character of a (virtual) representation. The claim shows that the class corresponding to $a(\tilde{\rho}) \cdot \delta_{\Pi}$ is an $R(G)$-module generator of $K_{G}^{\tau+n}(G)$. For the claim, first note that the element $\mu=\rho / \tilde{\rho}$ is $W$-invariant (and is in fact the square root of a character of $J$ ). It follows from the Weyl character formula that

$$
\frac{a\left(\left(\lambda \tilde{\rho}^{-1}\right) \cdot \rho\right)}{a(\rho)}
$$

is, up to sign, the character of an irreducible representation. But then

$$
\begin{aligned}
\frac{a\left(\lambda \tilde{\rho}^{-1} \rho\right)}{a(\rho)} & =\frac{a(\lambda \mu)}{a(\rho)}=\mu \frac{a(\lambda)}{a(\rho)} \\
& =\frac{a(\lambda)}{a\left(\mu^{-1} \rho\right)}=\frac{a(\lambda)}{a(\tilde{\rho})} .
\end{aligned}
$$

Corollary 4.38. Let $U \in K_{G}^{\tau+n}(G)$ be the class corresponding to a $(\tilde{\rho}) \cdot \delta_{\Pi}$. The map "multiplication by $U$ " is an isomorphism

$$
R(G) / I^{\tau} \rightarrow K_{G}^{\tau+n}(G)
$$

of $R(G)$-modules.

Proof: That the map factors through the quotient by $I^{\tau}$ is Corollary 4.35, and that it is surjective is Proposition 4.37. The result now follows from the fact that both sides are free of rank equal to the number of free $W$-orbits in $A_{\epsilon, \text { reg }}$ (ie, the number of Verlinde conjugacy classes).

As described at the end of 3.6 when $\tau$ is primitive the $R(G)$-module $K_{G}^{\tau+n}(G)$ acquires the structure of an $R(G)$-algebra

Corollary 4.39. When $\tau$ is primitive, there is a canonical algebra isomorphism

$$
K_{G}^{\tau+n}(G) \approx R(G) / I^{\tau} .
$$


Remark 4.40. When $\tau$ is primitive, the pushforward map $K_{G}^{\tau}(e) \rightarrow K_{G}^{\tau+n}$ is a ring homomorphism. Being primitive, the restriction of $\tau$ to $\{e\} / / G$ comes equipped with a trivialization and so $K_{G}^{\tau}(e) \approx R(G)$.

The isomorphisms of Corollaries 4.38 and 4.39 are proved after tensoring with the complex numbers in [21, where the distributions $\delta_{f}$ are related to the Kac numerator at $q=1$. We refer the reader to $\S 6$ and $\S 7$ of [21] for further discussion.

We conclude with a further computation which will be used in Part II. We consider the situation of this section in which $G=T$ is a torus of dimension $n$. The group $K_{T}^{\tau}(\{e\})$ is the free abelian group on $\Lambda^{\tau}$, and the pushforward map $K_{T}^{\tau}(\{e\}) \rightarrow K_{T}^{\tau+n}(T)$ is defined.

Proposition 4.41. The pushforward map

$$
i_{!}: K_{T}^{\tau}(\{e\}) \rightarrow K_{T}^{\tau+n}(T)
$$

sends the class corresponding to $\lambda \in \Lambda^{\tau}$ to the class in $K_{T}^{\tau+n}(T) \approx \operatorname{Hom}_{\Pi}\left(\Lambda^{\tau}, \mathbb{Z}(\epsilon)\right)$ corresponding to the distribution with Fourier expansion

$$
\lambda^{-1} \sum_{\pi \in \Pi} \epsilon(\pi) \pi^{-1} .
$$

Proof: The pushforward map is the composition of the Thom isomorphism

$$
K_{T}^{\tau+0}(\{e\}) \rightarrow K_{T}^{\tau+n}(\mathfrak{t}, \mathfrak{t} \backslash\{0\}) \approx K_{T}^{\tau+n}(T, T \backslash\{e\})
$$

with the restriction map

$$
K_{T}^{\tau+n}(T, T \backslash\{e\}) \rightarrow K_{T}^{\tau+n}(T) .
$$

We wish to compute these maps using the spectral sequence for relative twisted $K$-theory described at the end of 33.7 In order to do so, however, we need to replace $T \backslash\{e\}$ and $\mathfrak{t} \backslash\{0\}$ by the smaller $T \backslash B_{e}$ and $\mathfrak{t} \backslash\left\{B_{0}\right\}$, where $B_{e}$ and $B_{0}$ are small open balls containing $e$ and 0 respectively. This puts us in the situation described at the end of of 33.7 , where the sheaf $\mathcal{K}_{\text {rel }}^{\tau+t}$ works out to be the extension by zero of the restriction of $\mathcal{K}^{\tau+t}$ to $B_{e}$.

Applying the spectral sequence argument of this section to the pairs $\left(\mathfrak{t}, \mathfrak{t} \backslash B_{0}\right)$ and $\left(T, T \backslash B_{e}\right)$ gives isomorphisms

$$
\begin{aligned}
K_{T}^{\tau+n}\left(T, T \backslash B_{e}\right) & \approx \operatorname{hom}_{\Pi}\left(\Lambda^{\tau}, H_{c}^{n}\left(\mathfrak{t}, \mathfrak{t} \backslash B_{\Pi}\right) \otimes \mathbb{Z}(\epsilon)\right) \\
K_{T}^{\tau+n}\left(\mathfrak{t}, \mathfrak{t} \backslash B_{0}\right) & \approx \operatorname{hom}_{c}\left(\Lambda^{\tau}, H_{c}^{n}\left(\mathfrak{t}, \mathfrak{t} \backslash B_{0}\right) \otimes \mathbb{Z}(\epsilon)\right),
\end{aligned}
$$

where $B_{\Pi} \subset \mathfrak{t}$ is the inverse image of $B_{e}$ under the exponential map. The same argument identifies the restriction mapping (4.42) with the map induced by

$$
H_{c}^{n}\left(\mathfrak{t}, \mathfrak{t} \backslash B_{\Pi}\right) \rightarrow H_{c}^{n}(\mathfrak{t}),
$$

and the isomorphism

$$
K_{T}^{\tau+n}\left(T, T \backslash B_{e}\right) \approx K_{T}^{\tau+n}\left(\mathfrak{t}, \mathfrak{t} \backslash B_{0}\right)
$$

with the map

$$
\operatorname{Hom}_{\Pi}\left(\Lambda^{\tau}, H_{c}^{n}\left(\mathfrak{t}, \mathfrak{t} \backslash B_{\Pi}\right) \otimes \mathbb{Z}(\epsilon)\right) \rightarrow \operatorname{Hom}_{c}\left(\Lambda^{\tau}, H_{c}^{n}\left(\mathfrak{t}, \mathfrak{t} \backslash B_{0}\right) \otimes \mathbb{Z}(\epsilon)\right)
$$

which first forgets the $\Pi$-action and then uses

$$
H_{c}^{n}\left(\mathfrak{t}, \mathfrak{t} \backslash B_{\Pi}\right) \rightarrow H_{c}^{n}\left(\bar{B}_{0}, B_{0}\right) \approx H_{c}^{n}\left(\mathfrak{t}, \mathfrak{t} \backslash B_{0}\right) .
$$


Finally, the isomorphism

$$
K_{T}^{\tau+n}\left(\mathfrak{t}, \mathfrak{t} \backslash B_{0}\right) \approx K_{T}^{\tau}(\mathrm{pt}) \otimes K^{n}\left(\mathfrak{t}, \mathfrak{t} \backslash B_{0}\right)
$$

shows that the Thom isomorphism is simply the tensor product of the identity map with suspension isomorphism $K^{0}(\mathrm{pt}) \rightarrow K^{n}\left(\mathfrak{t}, \backslash B_{0}\right)$ (which uses the orientation of $\mathfrak{t})$. In terms of (4.43) this means that the Thom isomorphism

$$
K_{T}^{\tau}(\mathrm{pt}) \approx \operatorname{Hom}_{c}\left(\Lambda^{\tau}, \mathbb{Z}(\epsilon)\right) \rightarrow K_{T}^{\tau}\left(\mathfrak{t}, \mathfrak{t} \backslash B_{0}\right) \approx \operatorname{hom}_{c}\left(\Lambda^{\tau}, H_{c}^{n}\left(\mathfrak{t}, \mathfrak{t} \backslash B_{0}\right) \otimes \mathbb{Z}(\epsilon)\right),
$$

is simply the map derived from the suspension isomorphism

$$
H^{0}(\mathrm{pt}) \approx H_{c}^{n}\left(\mathfrak{t}, \mathfrak{t} \backslash B_{0}\right) .
$$

The result follows easily from this.

4.3. The action of $W_{\text {aff }}^{e}$ on $\mathrm{t}$. We summarize here some standard facts about about affine Weyl groups and conjugacy classes in $G$. Our basic references are 7 , 22. Recall that we have fixed a maximal torus $T$ of $G$. We write $\Lambda$ for the character group of $T$ and $R$ for the set of roots. Following Bourbaki, write $N(T, R)$ for the subgroup of $\mathfrak{t}$ consisting of elements on which the roots vanish modulo $2 \pi \mathbb{Z}$. There is a short exact sequence

$$
N(T, R) \longmapsto \Pi \rightarrow \pi_{1} G .
$$

Let $\mathcal{H}$ be the set of hyperplanes forming the diagram of $G$. Thus

$$
\mathcal{H}=\left\{H_{k, \alpha} \mid k \in \mathbb{Z}, \alpha \in R\right\}
$$

where

$$
H_{k, \alpha}=\{x \in \mathfrak{t} \mid \alpha(x)=2 \pi k\} .
$$

The collection $\mathcal{H}$ is locally finite in the sense that each $s \in \mathfrak{t}$ has a neighborhood meeting only finitely many hyperplanes in $\mathcal{H}$. The affine Weyl group is the group $W_{\text {aff }}$ be the group generated by reflections in the hyperplanes $H_{k, \alpha} \in \mathcal{H}$. It has the structure

$$
N(T, R) \rtimes W .
$$

Proposition 4.44. Let $x \in \mathfrak{t}$. The stabilizer of $x$ in $W_{\text {aff }}$ is the finite reflection group generated by reflections through the hyperplanes $H_{k, \alpha}$ containing $x$.

Write $W_{a f f}^{e}=L \rtimes W$. There is a short exact sequence

$$
W_{\text {aff }} \longmapsto W_{\text {aff }}^{e} \rightarrow \pi_{1} G .
$$

Proposition 4.46. Let $x \in \mathfrak{t}$. If $\pi_{1} G$ is torsion free, then the stabilizer of $x$ in $W_{\text {aff }}^{e}$ coincides with the stabilizer of $x$ in $W_{\text {aff }}$. It is therefore the finite reflection group generated by reflections through the hyperplanes $H_{k, \alpha}$ containing $x$.

Proof: Write $W_{x}$ for the stabilizer of $x$ in $W_{a f f}^{e}$. The image of $W_{x}$ in $\mathfrak{t} \rtimes W$ is conjugate to a subgroup of $W$, and so $W_{x}$ is finite. By assumption $\pi_{1} G$ has no nontrivial finite subgroups. The exact sequence (4.45) then shows that $W_{x} \subset W_{\text {aff }}$. The result then follows from Proposition 4.44.

Write $\mathbb{R}_{\geq 0}=[0, \infty)$.

Proposition 4.47. Suppose that $(W, V)$ is a finite reflection group. The orbit space $V / W$ is homeomorphic to $\mathbb{R}^{n_{1}} \times \mathbb{R}_{>0}^{n_{2}}$. The group $W$ is generated by $n_{2}$ reflections. In particular, if $W$ is non-trivial, then $n_{2} \neq 0$. 
Proof: This follows immediately from the Theorems on pages 20 and 24 of [11.

\section{Appendix A. Groupoids}

We remind the reader that we are assuming throughout this paper that, unless otherwise specified, all spaces are locally contractible, paracompact and completely regular. These assumptions implies the existence of partitions of unity 13 and locally contractible slices through actions of compact Lie groups [27, 28].

A.1. Definition and First Properties. A groupoid is a category in which all morphisms are isomorphism. We will consider groupoids in the category of topological spaces. Thus a groupoid $X=\left(X_{0}, X_{1}\right)$ consists of a space $X_{0}$ of objects, a space $X_{1}$ of morphisms, and map "identity map" $X_{0} \rightarrow X_{1}$, a pair of maps "domain" and "range" $X_{1} \rightarrow X_{0}$, an associative composition law $X_{1} \times_{X_{0}} X_{1} \rightarrow X_{1}$, and an "inverse" map $X_{1} \rightarrow X_{1}$. Write $X_{n}=X_{1} \times_{X_{0}} \cdots \times_{X_{0}} X_{1}$ for the space of $n$-tuples of composeable maps. Then the collection $\left\{X_{n}\right\}$ is a simplicial space. The $i^{\text {th }}$ face map

is given by

$$
d_{i}: X_{n} \rightarrow X_{n-1}
$$

$$
d_{i}\left(f_{1}, \ldots f_{n}\right)= \begin{cases}\left(f_{2}, \ldots, f_{n}\right) & i=0 \\ \left(f_{1}, \ldots, f_{i} \circ f_{i+1}, \ldots, f_{n}\right) & 0<i<n \\ \left(f_{1}, \ldots, f_{n-1}\right) & i=n\end{cases}
$$

Even though a groupoid is a special kind of simplicial space, we'll refer to the simplicial space as the nerve of $X=\left(X_{0}, X_{1}\right)$ and write $X_{\bullet}$. Finally, we let

$$
|X|=\coprod_{n} X_{n} \times \Delta^{n} / \sim
$$

denote the geometric realization of $X_{\bullet}$.

Example A.1. (cf Segal [30) Suppose that $G$ is a topological group acting on a space $X$. Then the pair $(G, X)$ forms a groupoid with space of objects $X$ and in which a morphism from $x$ to $y$ is an element of $g$ for which $g \cdot x=y$. In this case $X_{0}=X$ and $X_{1}=G \times X$. The composition law is given by the multiplication in $G$. We will write $X / / G$ for this groupoid.

Example A.2. (Segal 30]) Suppose that $X$ is a space and $\mathcal{U}=\left\{U_{i}\right\}$ is a covering of $X$. The nerve of the covering $\mathcal{U}$ is the nerve of a groupoid. Indeed, let $N_{\mathcal{U}}$ be the category whose objects are pairs $\left(U_{i}, x\right)$ with $U_{i} \in \mathcal{U}$ and $x \in U_{i}$ and in which a morphism from $\left(U_{i}, x\right)$ to $\left(U_{j}, y\right)$ is an element $w \in U_{i} \times_{X} U_{j}$ whose projection to $U_{i}$ is $x$ and whose projection to $U_{j}$ is $y$. Then $N_{\mathcal{U}}$ is a groupoid. If $U_{i}$ and $U_{j}$ are open subsets of $X$ then such a map exists if and only if $x=y$, in which case it is unique. Writing $X_{0}=\coprod U_{i}$, then $X_{n}=X_{0} \times_{X} \cdots \times_{X} X_{0}$, and the nerve of this groupoid is just the nerve of the covering $\mathcal{U}$.

Definition A.3. A map of groupoids $F: X \rightarrow Y$ is an equivalence if it is fully faithful and essentially surjective; that is, if every object $y \in Y_{0}$ is isomorphic to one of the form $F x$, and if for every $a, b \in X_{0}$ the map

$$
F: X(a, b) \rightarrow Y(F a, F b)
$$

is a homeomorphism. 
An equivalence of ordinary categories automatically admits an inverse (up to natural isomorphism). Examples A.6 and A.8 below show that the same is not necessarily true of an equivalence of topological categories, or groupoids. Requiring the existence of a globally defined inverse is, on the other hand too restrictive.

Definition A.4. A local equivalence $X \rightarrow Y$ is an equivalence of groupoids with the additional property that each $y \in Y_{0}$ has a neighborhood $U$ admitting a lift in the diagram

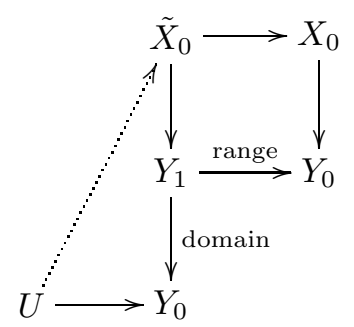

in which the square is Cartesian.

Remark A.5. The term local equivalence derives from thinking of a groupoid $X$ as defining a sheaf $U \mapsto X(U)$ on the category of topological spaces. An equivalence $X \rightarrow Y$ has a globally defined inverse if and only if for every space $U$ the map $X(U) \rightarrow Y(U)$ is an equivalence. As one easily checks, a map $X \rightarrow Y$ is a local equivalence if and only if it is an equivalence on stalks. We will say that two groupoids $X$ and $Y$ as being weakly equivalent, if there is a diagram of local equivalences

$$
X \leftarrow Z \rightarrow Y
$$

Example A.6. If $\mathcal{U}$ is an open covering of a space $X$, then the map

$$
N_{\mathcal{U}} \rightarrow X
$$

is a local equivalence. More generally, if $\mathcal{U} \rightarrow \mathcal{V}$ is a map of coverings of $X$, then

$$
N_{\mathcal{U}} \rightarrow N_{\mathcal{V}}
$$

is a local equivalence.

Example A.7. Given groupoids $X$ and $A$, write $X(A)$ for the groupoid of maps $A \rightarrow X$. Then a map $X \rightarrow Y$ is a local equivalence if and only if for spaces $S$, the map

$$
\underset{\mathcal{U}}{\lim _{\mathcal{U}}} X(U) \rightarrow \underset{\mathcal{U}}{\lim } Y(U)
$$

is an equivalence of groupoids, where $\mathcal{U}$ ranges over all coverings of $S$. Stated more succinctly, a map of groupoids is a local equivalence if and only if the corresponding map of presheaves of groupoids is a stalkwise equivalence.

Example A.8. If $P \rightarrow X$ is a principal $G$-bundle over $X$, then

$$
P / / G \rightarrow X
$$

is a local equivalence.

Example A.9. If $H \subset G$ is a subgroup, the map of groupoids

$$
\mathrm{pt} / / H \rightarrow(G / H) / / G
$$

is a local equivalence. 
The fiber product of functors

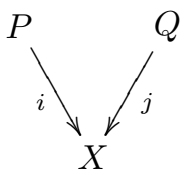

is the groupoid $P \times_{X} Q$ whose objects consist of

$$
p \in P, \quad q \in Q, \quad x \in X
$$

and isomorphisms

$$
i p \rightarrow x \leftarrow j q .
$$

The morphisms are the evident commutative diagrams. To give a functor $S \rightarrow$ $P \times{ }_{X} Q$ is to give a functors

$$
S \stackrel{p}{\rightarrow} P, \quad S \stackrel{q}{\rightarrow} Q, \quad S \stackrel{x}{\rightarrow} X
$$

and natural isomorphisms

$$
i \circ p \rightarrow x \leftarrow j \circ q .
$$

The groupoid $P \times_{X} Q$ is usually called fiber product of $P$ and $Q$ over $X$, even though strictly speaking it is a kind of homotopy fiber product and not the categorical fiber product. We will also say that the morphism $P \times_{X} Q \rightarrow Q$ is obtained from $P \rightarrow X$ by change of base along $j: Q \rightarrow X$. A natural transformation $T: j_{1} \rightarrow j_{2}$ gives a natural isomorphism between the groupoids obtained by change of base along $j_{1}$ and $j_{2}$.

One easily checks that the class of local equivalences is stable under composition and change of base. Consequently, if $P \rightarrow X$ and $Q \rightarrow X$ are both local equivalences, so is $P \times_{X} Q \rightarrow X$. Using Example A.7 one easily checks that if two of three maps in a composition are local equivalences so is the third.

Definition A.10. The 2-category $\underline{\operatorname{Cov}}_{X}$ is the category whose objects are local equivalences $p: P \rightarrow X$ and in which a 1-morphism from $p_{1}: P_{1} \rightarrow X$ to $p_{2}: P_{2} \rightarrow$ $X$ consists of a functor $F: P_{1} \rightarrow P_{2}$ and a natural transformation $T: p_{1} \rightarrow p_{2} \circ F$ making

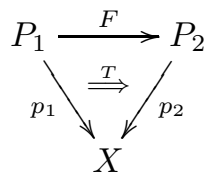

commute. A 2-morphism $\left(F_{1}, T_{1}\right) \rightarrow\left(F_{2}, T_{2}\right)$ is a natural transformation $\eta: F_{1} \rightarrow$ $F_{2}$ for which $T_{2}=p_{2} \eta \circ T_{1}$.

We will denote by $\operatorname{Cov}_{X}$ the 1-category quotient of $\underline{\operatorname{Cov}}_{X}$. The objects of $\operatorname{Cov} X$ are those of $\underline{\operatorname{Cov}}_{X}$, and $\operatorname{Cov}_{X}(a, b)$ is the set of isomorphism classes in $\underline{\operatorname{Cov}}_{X}(a, b)$. We will see that $\underline{\operatorname{Cov}}_{X}$ and $\operatorname{Cov}_{X}$ are not that different from each other.

Lemma A.11. For every $a, b \in \underline{\operatorname{Cov}}_{X}$, the category $\underline{\operatorname{Cov}}_{X}(a, b)$ is a codiscrete groupoid: there is a unique morphism between any two objects. 
Proof: Write $a=\left(F_{1}, T_{1}\right)$ and $b=\left(F_{2}, T_{2}\right)$

$$
P_{1} \underset{P_{1}}{\stackrel{F_{1}, F_{2}}{\longrightarrow}} P_{2} .
$$

A morphism (natural transformation) $\eta \in \underline{\operatorname{Cov}}_{X}(a, b)$ associates to $x \in P_{1}$ a map

$$
\eta_{x}: F_{1} x \rightarrow F_{2} x
$$

whose image

$$
p_{2} \eta_{x}: p_{2} F_{1} x \rightarrow p_{2} F_{2} x
$$

is prescribed to fit into the diagram of isomorphisms

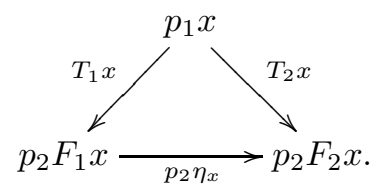

The map $p_{2} \eta_{x}$ is therefore forced to be $\left(T_{2} x\right) \circ\left(T_{1} x\right)^{-1}$, and so $\eta_{x}$ is uniquely determined since $P_{2} \rightarrow X$ is an equivalence.

Corollary A.12. The 1-category quotient $\operatorname{Cov}_{X}$ is a (co-)directed class.

Proof: Suppose that $p_{i}: P_{i} \rightarrow X, i=1,2$ are two objects of $\underline{\operatorname{Cov}}_{X}$. The groupoid $P_{12}=P_{1} \times_{X} P_{2}$ comes equipped with maps $P_{12} \rightarrow P_{1}$ and $P_{12} \rightarrow P_{2}$. If $f, g: P \rightarrow Q$ are two morphisms in $\underline{\mathrm{Cov}}_{X}$ there is, by Lemma A.11, a unique 2-morphism relating them, and so in fact $f=g$ in $\operatorname{Cov}_{X}$

A.2. Further Properties of Groupoids. We now turn to several constructions which are invariants of local equivalences. A groupoid is a presentation of a stack, and the invariants of local equivalences are in fact the invariants of the underlying stack.

A.2.1. Point set topology of groupoids. The orbit space or coarse moduli space of a groupoid $X$ is the space of isomorphism classes of objects, topologized as a quotient space of $X_{0}$. We denote the coarse moduli space of $X$ by $[X]$. At this level of generality, the space $[X]$ can be somewhat pathological, and without some further assumptions might not be in our class of locally contractible, paracompact, and completely regular spaces. When $X=S / / G$, then $[X]$ is the orbit space $S / G$, and in that case we will revert to the more standard notation $S / G$. A local equivalence $Y \rightarrow X$ gives a homeomorphism $[Y] \rightarrow[X]$.

For a subspace $S \subset[X]$ we denote $X_{S}$ the full sub-groupoid of $X$ consisting of objects in the isomorphism class of $S$. There is a one to one correspondence between full sub-groupoids $A \subset X$ containing every object in their $X$-isomorphism class and subspaces $[A]$ of $[X]$. With this we transport many notions from the point set topology of spaces to the context of groupoids. When $S$ is closed (resp. open) we will say that $X_{S}$ is a closed (resp. open) subgroupoid of $X$. We can speak of the interior and closure of a full subgroupoid. By an open covering of a groupoid, we mean an open covering $\left\{S_{\alpha}\right\}$ of $[X]$, in which case the collection $\left\{X_{S_{\alpha}}\right\}$ forms a covering of $X$ by open sub-groupoids. 
More generally, if $f: S \rightarrow[X]$ is a map, we can form a groupoid $X_{S}$ with objects the pairs $(s, x) \in S \times X_{0}$ for which $x$ is in the isomorphism class of $f(s)$. A map $(s, x) \rightarrow(t, y)$ is just a map from $x$ to $y$ in $X$. Phrased differently, the groupoid $X_{S}$ is the groupoid whose nerve fits into a pull-back square

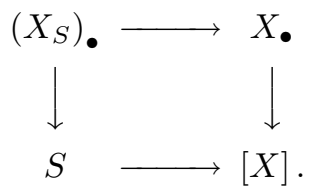

We will say that $X_{S}$ is defined by pullback from the map $S \rightarrow[X]$. With this we can transport many of the maneuvers of homotopy theory to the context of groupoids. For instance if $S=[X] \times I$, and $f$ is the projection, then $X_{S}$ is the groupoid $X \times I$. One can then form mapping cylinders and other similar constructions.

For example, suppose that $[X]$ is paracompact, and written as the union of two sets $S_{0}, S_{0}$ whose interiors cover. Write $U_{i}=X_{S_{i}}$. The $U_{i}$ are (full) subgroupoids whose interiors cover $X$. Let $N$ denote the groupoid constructed from the map

$$
\operatorname{cyl}\left(S_{0} \leftarrow S_{0} \cap S_{1} \rightarrow S_{1}\right) \rightarrow[X] .
$$

It is the double mapping cylinder of

$$
U_{0} \leftarrow U_{0} \cap U_{1} \rightarrow U_{1} .
$$

Following Segal [30], a partition of unity $\left\{\phi_{0}, \phi_{1}\right\}$ subordinate to the covering $\left\{S_{0}, S_{1}\right\}$, defines a map

$$
[X] \rightarrow \operatorname{cyl}\left(S_{0} \leftarrow S_{0} \cap S_{1} \rightarrow S_{1}\right) .
$$

The composite

$$
[X] \operatorname{cyl}\left(S_{0} \leftarrow S_{0} \cap S_{1} \rightarrow S_{1}\right) \rightarrow[X]
$$

is the identity, and so we get functors

$$
X \rightarrow N \rightarrow X
$$

whose composite is the identity. On the other hand, the composite

$$
\operatorname{cyl}\left(S_{0} \leftarrow S_{0} \cap S_{1} \rightarrow S_{1}\right)[X] \rightarrow \operatorname{cyl}\left(S_{0} \leftarrow S_{0} \cap S_{1} \rightarrow S_{1}\right)
$$

is homotopic to the identity, by a homotopy (the obvious linear homotopy) that preserves the map to $[X]$ (it is a homotopy in the category of spaces over $[X]$ ). This homotopy then defines by pullback, a homotopy

$$
N \times \Delta^{1} \rightarrow N
$$

from the composite $N \rightarrow X \rightarrow N$ to the identity map of $N$, fixing the map to $X$. In this way $X$ becomes a strong deformation retract of $N$, and $N$ is decomposed in a way especially well-suited for constructing sequences of Mayer-Vietoris type.

A groupoid $X$ has proper diagonal if the map

$$
X_{1} \stackrel{\text { (domain,range) }}{\longrightarrow} X_{0} \times X_{0}
$$

is proper, and $[X]$ is Hausdorff. If (A.13) is proper and $X_{0}$ is Hausdorff then $X$ is proper. If $Y \rightarrow X$ is a local equivalence, then $X$ has proper diagonal if and only if $Y$ has proper diagonal. 
A.2.2. Local and global quotients. A groupoid which is related by a chain of local equivalences to one of the form $S / / G$, obtained from a group $G$ acting on a space $S$, is said to be a global quotient. A local quotient groupoid is a groupoid $X$ admitting a countable open cover $\left\{U_{\alpha}\right\}$ with the property that each $X_{U_{\alpha}}$ is weakly equivalent to a groupoid of the form $S / / G$ with $G$ a compact Lie group, and $S$ a Hausdorff space. If $Y \rightarrow X$ is a local equivalence, then $Y$ is a local quotient groupoid if and only if $X$ is, so the property of being a local quotient is intrinsic to the underlying stack.

If $X$ is a local quotient groupoid, then $[X]$ is paracompact, locally contractible and completely regular. If $X$ is a local quotient groupoid with the property that there is at most one map between any two objects (ie $X_{1} \rightarrow X_{0} \times X_{0}$ is an inclusion), the map $X \rightarrow[X]$ is a local equivalence, and so $X$ is just a space.

The following lemma is straightforward.

Lemma A.14. Any groupoid constructed by pullback from a local quotient groupoid is a local quotient groupoid. In particular, any (full) subgroupoid of a local quotient groupoid is a local quotient groupoid, and the mapping cylinder of a map $X_{S} \rightarrow X$ constructed by pullback along a map $S \rightarrow[X]$ to the orbit space of a local quotient groupoid is a local quotient groupoid.

A.3. Fiber bundles over groupoids and descent. In this section we define the category of fiber bundles over a groupoid, and show that a local equivalence gives an equivalence of categories of fiber bundles (Proposition A.18). Thus the category of fiber bundles over a groupoid is intrinsic to the underlying stack.

A fiber bundle over a groupoid $X=\left(X_{0}, X_{1}\right)$ consists of a fiber bundle $P$ on $X_{0}$ together with identifications of certain pullbacks to $X_{n}$ for various $n$. We introduce some convenient notation for describing these pulled back bundles.

Let's denote a typical point of $X_{n}$ by

$$
x_{0} \stackrel{f_{1}}{\longrightarrow} \ldots \stackrel{f_{n}}{\longrightarrow} x_{n} .
$$

Given a bundle $P \rightarrow X_{0}$ we'll write $P_{x_{i}}$ for the pullback of $P$ along the map

$$
\begin{aligned}
X_{n} & \rightarrow X_{0} \\
\left(x_{0} \rightarrow \cdots \rightarrow x_{n}\right) & \mapsto x_{i} .
\end{aligned}
$$

Similarly, if $P \rightarrow X_{1}$ is given, we'll write $P_{f_{i}}$ for the pullback of $P$ along the map

$$
\begin{aligned}
X_{n} & \rightarrow X_{1} \\
\left(x_{0} \stackrel{f_{1}}{\longrightarrow} \ldots \stackrel{f_{n}}{\longrightarrow} x_{n}\right) & \mapsto\left(x_{i-1} \stackrel{f_{i}}{\longrightarrow} x_{i}\right),
\end{aligned}
$$

and $P_{f_{i} \circ f_{i+1}}$ for the pullback along

$$
\begin{aligned}
X_{n} & \rightarrow X_{1} \\
\left(x_{0} \stackrel{f_{1}}{\longrightarrow} \ldots \stackrel{f_{n}}{\longrightarrow} x_{n}\right) & \mapsto\left(x_{i-1} \stackrel{f_{i} \circ f_{i+1}}{\longrightarrow} x_{i+1}\right),
\end{aligned}
$$

etc. For small values of $n$ we'll use symbols like

$$
\begin{gathered}
(a \stackrel{f}{\rightarrow} b) \in X_{1} \\
(a \stackrel{f}{\rightarrow} b \stackrel{g}{\rightarrow} c) \in X_{2} .
\end{gathered}
$$

to denote typical points. 
Definition A.15. A fiber bundle on $X$ consists of a fiber bundle $P \rightarrow X_{0}$, together with an bundle isomorphism

$$
t_{f}: P_{a} \rightarrow P_{b}
$$

on $X_{1}$, for which $t_{\mathrm{Id}}=\mathrm{Id}$, and satisfying the cocycle condition that

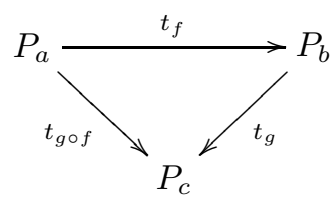

commutes on $X_{2}$.

This way of describing a fiber bundle is convenient when thinking of $X$ as a category. The association $a \rightarrow P_{a}$ is a functor from $X$ to spaces, that is continuous in an appropriate sense. There is a more succinct way of describing a fiber bundle on a groupoid. Namely, a fiber bundle on a groupoid $X=\left(X_{0}, X_{1}\right)$ is a groupoid $P=\left(P_{0}, P_{1}\right)$ and a functor $P \rightarrow X$ making $P_{i} \rightarrow X_{i}$ into fiber bundles, and all of the structure maps into maps of fiber bundles (i.e., pullbacks squares).

A functor $F: Y \rightarrow X$ between groupoids defines, in the evident way, a pullback functor $F^{*}$ from the category of fiber bundles over $X$ to the category of fiber bundles over $Y$. A natural transformation $T: F \rightarrow G$ defines a natural transformation $T^{*}: F^{*} \rightarrow G^{*}$

Example A.17. Let $\mathcal{U}=\left\{U_{i}\right\}$ be a covering of a space $X$. To give a fiber bundle over $N_{\mathcal{U}}$ is to give a fiber bundle $P_{i}$ on each $U_{i}$ and the clutching (descent) data needed to assemble the $P_{i}$ into a fiber bundle over $X$. Indeed, pullback along the map $N_{\mathcal{U}} \rightarrow X$ gives an equivalence between the category of fiber bundles over $X$ and the category of fiber bundles over $N_{\mathcal{U}}$.

The following generalization of Example A.17 will be referred to as descent for fiber bundles over groupoids.

Proposition A.18. Suppose that $F: X \rightarrow Y$ is a local equivalence. Then the pullback functor

$$
F^{*}:\{\text { Fiber bundles on } Y\} \rightarrow\{\text { Fiber bundles on } X\}
$$

is an equivalence of categories.

Proof: Suppose that $P$ is a fiber bundle over $Y$, which we think of as a functor from $Y$ to the category of topological spaces. Since $Y \rightarrow X$ is an equivalence of categories, the functor $F^{*}$ has a left adjoint $F_{*}$, given by

$$
F_{*} P(x)=\underset{Y / x}{\lim _{Y / x}} P
$$

where $Y / x$ is the category of objects in $y \in Y$ equipped with a morphism $F y \rightarrow x$. Since $Y \rightarrow X$ is an equivalence of groupoids, there is a unique map between any two objects of $Y / x$, and so $F_{*} P(x)$ is isomorphic to $P_{y}$ for any $y \in Y / x$. For each $x \in X$, choose a neighborhood $x \in U \subset X_{0}$, a map $t: U \rightarrow Y_{0}$, and a family of morphisms $U \rightarrow X_{1}$ connecting $F \circ t$ to the inclusion $U \rightarrow X_{0}$. We topologize

$$
\bigcup_{x \in X} F_{*} P_{x}
$$


by requiring that the canonical map

$$
\left.t^{*} P \rightarrow F_{*} P\right|_{U}
$$

be a homeomorphism. This gives $F_{*} P$ the structure of a fiber bundle over $X_{0}$. Naturality provides $F_{*} P$ with the additional structure required to make it into a fiber bundle over $X$. One easily checks that the pair $\left(F_{*}, F^{*}\right)$ is an adjoint equivalence of the category of fiber bundles over $X$ with the category of fiber bundles over $Y$.

For a fiber bundle $p: P \rightarrow X$ write $\Gamma(P)$ for the space of sections

$$
\Gamma(P)=\Gamma(X ; P)=\left\{s: X \rightarrow P \mid p \circ s=\operatorname{Id}_{X}\right\},
$$

topologized as a subspace of

$$
X_{0}^{P_{0}} \times X_{1}^{P_{1}}
$$

If $f: Y \rightarrow X$ is a local equivalence, and $P \rightarrow X$ is a fiber bundle, then the evident map

$$
\Gamma(X ; P) \rightarrow \Gamma\left(Y ; f^{*} P\right)
$$

is a homeomorphism.

If $P$ is a pointed fiber bundle, with $s: X \rightarrow P$ as a basepoint, and $A \subset X$ is a (full) subgroupoid, write $\Gamma(X, A ; P)$ for the space of section $x$ of $P$ for which $\left.x\right|_{A}=s$.

Now suppose that $P \rightarrow Q$ is a map of fiber bundles over $X$, and $\left\{U_{\alpha}\right\}$ is a covering of $X$ by open sub-groupoids. Write $P_{\alpha} \rightarrow U_{\alpha}$ for the restriction of $P$ to $U_{\alpha}$, and $P_{\alpha_{1}, \ldots, \alpha_{n}}$ for the restriction of $P$ to $U_{\alpha_{1}} \cap \cdots \cap U_{\alpha_{n}}$, and similarly for $Q$.

Proposition A.19. If for each non-empty finite collection $\left\{\alpha_{1} \ldots \alpha_{n}\right\}$ the map

$$
\Gamma\left(P_{\alpha_{1}, \ldots, \alpha_{n}}\right) \rightarrow \Gamma\left(Q_{\alpha_{1}, \ldots, \alpha_{n}}\right)
$$

is a weak homotopy equivalence, then so is

$$
\Gamma(P) \rightarrow \Gamma(Q) .
$$

Proof: This is a straightforward application of the techniques of Segal [30]. Let's first consider the case in which $X$ is covered by just two open sub-groupoids $U$ and $V$. We form the "double mapping cylinder"

$$
C=U \amalg U \cap V \times[0,1] \amalg V / \sim,
$$

and consider the functor $g: C \rightarrow X$. A choice of partition unity on $[X]$ subordinate to the covering $\{[U],[V]\}$ gives a section of $g$ making $\Gamma(X ; P) \rightarrow \Gamma(X ; Q)$ a retract of $\Gamma\left(C ; g^{*} P\right) \rightarrow \Gamma\left(C ; g^{*} Q\right)$. It therefore suffices, in this case, to show that $\Gamma\left(C ; g^{*} P\right) \rightarrow \Gamma\left(C ; g^{*} Q\right)$ is a weak equivalence. But $\Gamma\left(C ; g^{*} P\right)$ fits into a homotopy pullback square

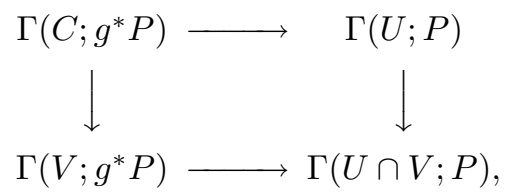

and similarly for $\Gamma\left(C ; g^{*} Q\right)$ (to simplify the diagram we have not distinguished in notation between $P$ and its restriction to $U, V$, and $U \cap V)$. The result then follows from the long exact (Mayer-Vietoris) sequence of homotopy groups. An easy induction then gives that the map on spaces of sections of $P \rightarrow Q$ restricted to any finite union $U_{\alpha_{1}} \cup \cdots \cup U_{\alpha_{n}}$ is a weak equivalence. For the case the collection 
$\left\{U_{\alpha}\right\}$ is countable (to which we are reduced when $[X]$ is second countable), order the $U_{\alpha}$ and write

$$
V_{n}=U_{1} \cup \cdots \cup U_{n}
$$

Form the "infinite mapping cylinder"

$$
C=\coprod V_{i} \times[i, i+1] / \sim,
$$

and consider $g: C \rightarrow X$. As before, a partition of unity on $[X]$ subordinate to the covering $[V]_{i}$ defines a section of $[C] \rightarrow[X]$ and hence of $C \rightarrow X$, making $\Gamma(X ; P) \rightarrow \Gamma(X ; Q)$ a retract of

$$
\Gamma\left(C ; g^{*} P\right) \rightarrow \Gamma\left(C ; g^{*} Q\right) .
$$

It therefore suffices to show that A.20 is a weak equivalence. But A.20 is the homotopy inverse limit of the tower

$$
\Gamma\left(V_{n} ; P\right) \rightarrow \Gamma\left(V_{n} ; Q\right)
$$

and so its homotopy groups (or sets, in the case of $\pi_{0}$ ) are related to those of (A.21) by a Milnor sequence, and the result follows.

Alternatively, following Segal [30, one can avoid the countability hypothesis and the induction by using for $C$ the nerve of the covering $\left\{U_{\alpha}\right\}$ and the homotopy spectral sequences of Bousfield-Kan and Bousfield [9, 8.

A.4. Hilbert bundles. A Hilbert bundle over a groupoid $X$ is a fiber bundle whose fibers have the structure of a separable $\mathbb{Z} / 2$-graded Hilbert space.

Remark A.22. There is a tricky issue in the point set topology here. In defining Hilbert bundles as special kinds of fiber bundles, we're implicitly using the compact open topology on $U(H)$ and not the norm topology. This causes trouble when we form the associated bundle of Fredholm operators ( the norm topology on the space of Fredholm operators. This issue is raised and resolved by Atiyah-Segal [3], and we are following their discussion in this paper.

Definition A.23. A Hilbert bundle $H$ is universal if for each Hilbert bundle $V$ there exists a unitary embedding $V \subset H$. The bundle $H$ is said to have the absorption property if for any $V$, there is a unitary equivalence $H \oplus V \approx H$.

Lemma A.24. A universal Hilbert bundle has the absorption property.

Proof: First note that if $H$ is universal, then

$$
H \otimes \ell^{2} \approx H \oplus H \oplus \cdots
$$

has the absorption property. Indeed, given $V$ write $H=W \oplus V$, and use the "Eilenberg swindle"

$$
\begin{aligned}
V \oplus H \oplus H \oplus \cdots & \approx V \oplus(W \oplus V) \oplus(W \oplus V) \oplus \cdots \\
& \approx(V \oplus W) \oplus(V \oplus W) \oplus(V \oplus W) \cdots \approx H \oplus H \oplus H \oplus \cdots .
\end{aligned}
$$

We can then write $H \approx H \otimes \ell^{2} \oplus V \approx H \otimes \ell^{2}$, to conclude that $H$ is absorbing.

Definition A.25. A Hilbert bundle $H$ over $X$ is locally universal if for every open sub-groupoid $X_{U} \subset X$ the restriction of $H$ to $X_{U}$ is universal. 
Lemma A.26. If $H$ and $H^{\prime}$ are universal Hilbert bundles on $X$, then there is a unitary equivalence $H \approx H^{\prime}$.

Remark A.27. Since the category of Hilbert bundles on $X$ depends only on $X$ up to local equivalence, if $f: Y \rightarrow X$ is a local equivalence and $H$ is a (locally) universal Hilbert bundle on $X$, then $f^{*} H$ is a (locally) universal Hilbert bundle on $Y$. Similarly, if $H^{\prime}$ is a (locally) universal Hilbert bundle on $Y$ there is a (locally) universal Hilbert bundle $H$ on $X$, and a unitary equivalence $f^{*} H \approx H^{\prime}$.

We now show that the existence of a locally universal Hilbert bundle is a local issue.

Lemma A.28. Suppose that $X$ is a groupoid, and that

$$
\left\{U_{i} \mid i=1 \ldots \infty\right\}
$$

is a covering of $X$ by open sub-groupoids. If $H$ is a Hilbert bundle with the property that

$$
H_{i}=\left.H\right|_{U_{i}}
$$

is universal, then $H \otimes \ell^{2}$ is universal.

Proof: Let $V$ be a Hilbert-space bundle on $X$. Choose a partition of unity $\left\{\lambda_{i}\right\}$ on $[X]$ subordinate to the open cover $[U]_{i}$. For each $i$ choose an embedding

$$
r_{i}:\left.V\right|_{U_{i}} \hookrightarrow H_{i} .
$$

The map

$$
V \rightarrow H \oplus H \oplus \cdots=H \otimes \ell^{2}
$$

with components $\lambda_{i} r_{i}$ is then an embedding of $V$ in $H \otimes \ell^{2}$.

Corollary A.29. In the situation of Lemma A.28, if $\left.H\right|_{U_{i}}$ is locally universal, then $H \otimes \ell^{2}$ is locally universal.

Lemma A.30. Suppose that $X$ is a groupoid, and that

$$
\left\{U_{i} \mid i=1 \ldots \infty\right\}
$$

is a covering of $X$ by open sub-groupoids. If $H_{i}$ is a locally universal Hilbert bundle on $\left\{U_{i}\right\}$, then there exists a Hilbert bundle $H$ on $X$ with $\left.H\right|_{U_{i}} \approx H_{i}$.

Proof: This is an easy induction, using Lemma A.26

Corollary A.31. Suppose that $X$ is a groupoid, and that

$$
\left\{U_{i} \mid i=1 \ldots \infty\right\}
$$

is a covering of $X$ by open sub-groupoids. If $H_{i}$ is a locally universal Hilbert bundle on $\left\{U_{i}\right\}$, then there exists a locally universal Hilbert bundle $H$ on $X$.

Lemma A.32. Suppose that $X=S / / G$ is a global quotient of a space $S$ by a compact Lie group $G$. Then the equivariant Hilbert bundle $S \times L^{2}(G) \otimes C_{1} \otimes \ell^{2}$ is a locally universal Hilbert bundle on $X$. 
Here $C_{1}$ is the complex Clifford algebra on one (odd) generator. It is there simply to make the odd component of our Hilbert bundle large enough.

Proof: Since the open (full) subgroupoids of $S / / G$ correspond to the $G$-stable open subsets of $S$ it suffices to show that that $L^{2}(G) \otimes C_{1} \otimes \ell^{2}$ is universal. Let $V$ be any Hilbert bundle on $S / / G$, ie an equivariant Hilbert bundle on $S$. By Kuiper's theorem, $V$ is trivial as a (non-equivariant) Hilbert bundle on $S$. Choose an orthonormal homogeneous basis $\left\{e_{i}\right\}$, and let $e^{i}=\left\langle e_{i},-\right\rangle: V \rightarrow C_{1}$ be the corresponding projection operator. By the universal property of $L^{2}(G)$, each $e^{i}$ lifts uniquely to an equivariant map

$$
V \rightarrow L^{2}(G) \otimes C_{1} .
$$

Taking the sum of these maps gives an embedding of $V$ in $L^{2}(G) \otimes C_{1} \otimes \ell^{2}$.

Combining Lemma A.32 with Lemma A.30 gives:

Corollary A.33. If $X$ is a local quotient groupoid, then there exists a locally universal Hilbert bundle on $X$.

Corollary A.34. Suppose that $X$ is a local quotient groupoid, $f: Y \rightarrow X$ is a map constructed by pullback from $[Y] \rightarrow[X]$. If $H$ is locally universal on $X$, then $f^{*} H$ is locally universal on $Y$.

Proof: This is an easy consequence of Lemma A.32 and Corollary A.29,

Corollary A.34 is needed is the proof of excision in twisted $K$-theory, and is the reason for our restriction to the class of local quotient groupoids.

The following result is well-known, but we could not quite find a reference. Our proof is taken from [25. Theorem 1.5] which gives the analogous result for equivariant embeddings of countably infinite dimensional inner product spaces (and not Hilbert spaces). Of course the result also follows from Kuiper's theorem, since the space of embeddings is $U\left(H \otimes \ell^{2}\right) / U\left(V^{\perp}\right)$. But the contractibility of the space of embeddings is more elementary than the contractibility of the unitary group, so it seemed better to have proof that doesn't make use of Kuiper's theorem.

Lemma A.35. Suppose that $V$ and $H$ are Hilbert bundles over a groupoid $X$, and that there is a unitary embedding $V \subset H \otimes \ell^{2}$. Then the space of embeddings $V \hookrightarrow H \otimes \ell^{2}$ is contractible.

Proof: Let $f: V \subset H \otimes \ell^{2}$ be a fixed embedding, and write

$$
\begin{aligned}
H \otimes \ell^{2} & =H \oplus H \oplus \cdots \\
f & =\left(f_{1}, f_{2}, \ldots\right) .
\end{aligned}
$$

The contraction is a concatenation of two homotopies. The first takes an embedding

$$
g=\left(g_{1}, g_{2}, \ldots\right)
$$

to

$$
\left(0, g_{1}, 0, g_{2}, \ldots\right)
$$

and then the second is

$$
\cos (\pi t / 2) \cdot\left(0, g_{1}, 0, g_{2}, \ldots\right)+\sin (\pi t / 2) \cdot\left(f_{1}, 0, f_{2}, 0 \ldots\right) .
$$


It is easier to write down the reverse of the first homotopy. It, in turn, is the concatenation of an infinite sequence of 2-dimensional rotations

$$
\begin{array}{ccrl}
\left(0, g_{1}, 0, g_{2}, 0, g_{3}, \ldots\right) & \mapsto\left(g_{1}, 0,0, g_{2}, 0, g_{3}, \ldots\right) & & 0 \leq t \leq 1 / 2 \\
\left(g_{1}, 0,0, g_{2}, 0, g_{3}, \ldots\right) & \mapsto\left(g_{1}, g_{2}, 0,0,0, g_{3}, \ldots\right) & & 1 / 2 \leq t \leq 3 / 4 \\
& \cdots & &
\end{array}
$$

One must check that the limit as $t \mapsto 1$ is $\left(g_{1}, g_{2}, \ldots\right)$, and the that path is continuous in the compact-open topology. Both facts are easy and left to the reader.

Lemma A.36. Suppose that $H$ is a locally universal Hilbert bundle over a local quotient groupoid $X$. Then the space of sections $\Gamma(X ; U(H))$ of the associated bundle of unitary groups is weakly contractible.

Proof: This follows easily from Kuiper's theorem (see Appendix 3 of [3]), and Proposition A.19.

We conclude this section with a useful criterion for a local-quotient stack to be equivalent to a global quotient by a compact Lie group.

Proposition A.37. The (locally) universal Hilbert bundle over a compact, localquotient groupoid, splits into a sum of finite-dimensional bundles iff the groupoid is equivalent (in the sense of local equivalence) to one of the form $X / / G$, with $X$ compact, and $G$ a compact group.

Remark A.38. (i) This implies right away that the extensions of groupoids corresponding to twistings whose invariant in $H^{3}$ has infinite order are not quotient stacks: indeed, any 1-eigenbundle for the central $\mathbb{T}$ is a projective bundle representative for the twisting, and hence must be infinite-dimensional.

(ii) There are simple obstructions to a groupoid being related by a chain of local equivalences to a global quotient by a compact group; for instance, such quotients admit continuous choices of Ad-invariant metrics on the Lie algebra stabilizers which are integral on the co-weight lattices. The stack obtained by gluing the boundaries of $B(\mathbb{T} \times \mathbb{T}) \times[0,1]$ via the shearing automorphism of $\mathbb{T} \times \mathbb{T}$ does not carry such metrics. The same is true for the quotient stack $\mathcal{A} / / \mathbb{T} \ltimes L T$, where $T$ is a torus, and $\mathcal{A}$ is the space of connections on the trivial $T$-bundle over the circle. In this case, the stack is fibered over $T$ in $B(\mathbb{T} \times T)$-stacks with the tautological shearing holonomies. Hence, the larger stacks $\mathcal{A} / / \mathbb{T} \ltimes L G$ where $G$ is a compact Lie group and $\mathcal{A}$ is the space of connections on the trivial $G$-bundle over the circle are not global quotients either.

(iii) The result is curiously similar to Totaro's characterisation of smooth quotient stacks as the Artin stacks where coherent sheaves admit resolutions by vector bundles [32].

Proof. The 'if' part follows from our construction of the universal Hilbert bundle. For the 'only if part,' first note that a local quotient groupoid $X$ is weakly equivalent to a groupoid of the form $S / / G$, if and only if there is a principal $G$-bundle $P \rightarrow X$ with the property that there is at most one map between any two objects in $P$. In that case $P$ is equivalent to $[P]($ A.2.2), and $X$ is weakly equivalent to $[P] / / G$. This latter condition holds if and only if for each $x \in X_{0}$ the map $\operatorname{Aut}(x) \rightarrow G$ associated to $P$ is a monomorphism. Suppose that $H$ is the (locally) universal 
Hilbert bundle on $X$, and that we can find an orthogonal decomposition $H=\oplus H_{\alpha}$ with each $H_{\alpha}$ of dimension $n_{\alpha}<\infty$. Take $P$ to be the product of the frame bundles of the $H_{\alpha}$, and $G$ to be the product of the unitary groups $U\left(H_{\alpha}\right) \approx U\left(n_{\alpha}\right)$. To check that $\operatorname{Aut}(x) \rightarrow G$ is a monomorphism in this case it suffices to check locally near $x$. The assertion is thus reduced to the case of a global quotient by a compact group, where it follows from our explicit construction.

A.5. Fredholm operators and $K$-theory. We will build our model of twisted $K$-theory using the "skew-adjoint Fredholm" model of Atiyah-Singer [1. In this section we recall this theory, and the modifications described in Atiyah-Segal [3]

Let $H$ be a $\mathbb{Z} / 2$-graded Hilbert bundle over a groupoid $X$. We wish to associate to $H$ a bundle of Fredholm operators over $X$. As mentioned in Remark A.22 we cannot just use the norm topology on the space of Fredholm operators here. We have used the compact-open topology on $U(H)$, and the (conjugation) action of $U(H)$ in the compact-open topology on Fredholm in the norm topology is not continuous. Following Atiyah-Segal [3, Definition 3.2], we make the following definition.

Definition A.39. (3] $)$ The space Fred $^{(0)}(H)$ is the space of odd skew-adjoint Fredholm operators $A$, for which $A^{2}+1$ is compact, topologized as a subspace of $\mathcal{B}(H) \times \mathcal{K}(H)$, with $\mathcal{B}(H)$ given the compact-open topology and $\mathcal{K}(H)$ the norm topology.

Let $C_{n}=T\left\{\mathbb{C}^{n}\right\} /\left(z^{2}+q(z)=0\right)$ denote the complex Clifford algebra associated to the quadratic form $q(z)=\sum z_{i}^{2}$. We write $\epsilon_{i}$ for the $i^{\text {th }}$ standard basis element of $\mathbb{C}^{n}$, regarded as an element of $C_{n}$. Following Atiyah-Singer [1, for an operator $A \in$ Fred $\left.^{(()} C_{n} \otimes H\right)$, with $n$ odd, let

$$
w(A)= \begin{cases}\epsilon_{1} \ldots \epsilon_{n} A & n \equiv-1 \bmod 4 \\ i^{-1} \epsilon_{1} \ldots \epsilon_{n} A & n \equiv 1 \bmod 4 .\end{cases}
$$

The operator $A$ is then even and self-adjoint.

Definition A.40. ([3]) The space Fred ${ }^{(n)}(H)$ is the subspace of Fred $^{(0)}\left(C_{n} \otimes H\right)$ consisting of odd operators $A$ which commute (in the graded sense) with the action of $C_{n}$, and for which the essential spectrum of $w(A)$, in case $n$ is odd, contains both positive and negative eigenvalues.

Atiyah and Segal [3] show that the "identity" map from Fred ${ }^{(n)}\left(\ell^{2}\right)$ in the norm topology to $\operatorname{Fred}^{(n)}\left(\ell^{2}\right)$ in the above topology is a weak homotopy equivalence. It then follows from Atiyah-Singer [1, Theorem B(k)] that the map

$$
\begin{aligned}
\operatorname{Fred}^{(n)}\left(\ell^{2}\right) & \rightarrow \Omega^{\prime} \operatorname{Fred}^{(n-1)}\left(C_{1} \otimes \ell^{2}\right) \\
A & \mapsto \epsilon_{k} \cos (\pi t)+A \sin (\pi t)
\end{aligned}
$$

is weak homotopy equivalence, where we are making the evident identification $C_{n} \approx$ $C_{n-1} \otimes C_{1}$, and $\Omega^{\prime}$ denotes the space of paths from $\epsilon_{k}$ to $-\epsilon_{k}$. Combining these leads to the following simple consequence.

Proposition A.41. If $X$ is a local quotient groupoid, and $H$ a $\mathbb{Z} / 2$-graded, locally universal Hilbert bundle over $X$, the map

$$
\Gamma\left(X ; \text { Fred }^{(n+1)}(H)\right) \rightarrow \Omega^{\prime} \Gamma\left(X ; \operatorname{Fred}^{(n)}(H)\right)
$$

is a weak homotopy equivalence. 
Proof: By Proposition A.19, the question is local in $X$, so we may assume $X=S / / G$, with $G$ a compact Lie group. By our assumption on the existence of locally contractible slices, we may reduce to the case in which $S$ is equivariantly contractible to a fixed point $s \in S$. Finally, since the question is homotopy invariant in $X$, we reduce to the case $S=$ pt. We are therefore reduced to showing that if $H$ is a universal $G$-Hilbert space, then the map of $G$-fixed points

$$
\text { Fred }^{(n)}(H)^{G} \rightarrow \Omega^{\prime} \text { Fred }^{(n+1)}(H)^{G}
$$

is a weak equivalence. For each irreducible representation $V$ of $G$, let $H_{V}$ denote the $V$-isotypical component of $H$. Then $(\mathrm{A} .42$ is the product over the irreducible representations $V$ of $G$, of

$$
\text { Fred }^{(n)}\left(H_{V}\right)^{G} \rightarrow \Omega^{\prime} \text { Fred }^{(n+1)}\left(H_{V}\right)^{G}
$$

Since $H$ is universal, the Hilbert space $H_{V}$ is isomorphic to $V \otimes \ell^{2}$, and the map

$$
\begin{aligned}
\operatorname{Fred}^{(n)}\left(\ell^{2}\right) & \rightarrow \operatorname{Fred}^{(n)}\left(V \otimes \ell^{2}\right)^{G} \\
T & \mapsto \operatorname{Id} \otimes T
\end{aligned}
$$

is a homeomorphism. The Proposition is thus reduced to the result of Atiyah-Singer quoted above.

We now assemble the spaces $\Gamma\left(X ; \operatorname{Fred}^{(n)}(H)\right)$ into a spectrum in the sense of algebraic topology. To do this requires specifying basepoints in $\operatorname{Fred}^{(n)}(H)$. Since our operators are odd, we can't take the identity map as a basepoint and a different choice must be made. There are some technical difficulties that arise in trying to specify consistent choices and we have just chosen to be unspecific on this point. The difficulties don't amount to a serious problem since any invertible operator can be taken as a basepoint, and the space of invertible operators is contractible. The reader is referred to 23 for further discussion.

We will use the symbol $\epsilon$ to refer to a chosen basepoint in $\operatorname{Fred}^{(n)}(H)$, as well as to the constant section with value $\epsilon$ in $\Gamma\left(X ; \operatorname{Fred}^{(n)}(H)\right)$.

Proposition A.41 gives a homotopy equivalence

$$
\Gamma\left(X ; \operatorname{Fred}^{(n+1)}(W)\right) \rightarrow \Omega \Gamma\left(X ; \operatorname{Fred}^{(n)}(W)\right)
$$

As described in [1], the fact that $C_{2}$ is a matrix algebra gives a homeomorphism

$$
\Gamma\left(X \text { Fred }^{(m)}(W)\right) \approx \Gamma\left(X \text { Fred }^{(m+2)}(W)\right) .
$$

We the spectrum $\underline{K}(X)$ by taking

$$
\underline{K}(X)_{n}= \begin{cases}\Gamma\left(X, \text { Fred }^{(0)}(W)\right) & n \text { even } \\ \Gamma\left(X, \operatorname{Fred}^{(1)}(W)\right) & n \text { odd }\end{cases}
$$

with structure map $\underline{K}(X)_{n} \rightarrow \Omega \underline{K}(X)_{n+1}$ to be the map A.44 when $n$ is odd,

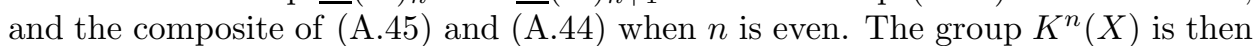
defined by

$$
K^{n}(X)=\pi_{0} \underline{K}(X)_{n} \approx \pi_{k} \underline{K}(X)_{n+k} .
$$

Because $H$ is locally universal, when $X=S / / G$, we have

$$
K^{n}(X) \approx\left[S, \text { Fred }^{(n)}\left(L^{2}(G) \otimes \ell^{2}\right)\right]_{G} .
$$


Since, as remarked in 3.5 .4 Fred $^{(n)}\left(L^{2}(G) \otimes \ell^{2}\right)$ is a classifying space for equivariant $K$-theory, this latter group can be identified with

$$
K^{n}(G)(S) .
$$

\section{REFERENCES}

[1] M. F. Atiyah and I. M. Singer, Index theory for skew-adjoint Fredholm operators, Inst. Hautes Études Sci. Publ. Math. (1969), no. 37, 5-26. MR 44 \#2257

[2] Michael Atiyah and Graeme Segal, Twisted K-theory and cohomology.

[3] — Twisted K-theory, Ukr. Mat. Visn. 1 (2004), no. 3, 287-330. MR MR2172633

[4] Roman Bezrukavnikov, Michael Finkelberg, and Ivan Mirković, Equivariant homology and K-theory of affine Grassmannians and Toda lattices, Compos. Math. 141 (2005), no. 3, 746-768. MR MR2135527 (2006e:19005)

[5] Raoul Bott, The space of loops on a Lie group, Michigan Math. J. 5 (1958), 35-61. MR MR0102803 (21 \#1589)

[6] Raoul Bott and Hans Samelson, Applications of the theory of Morse to symmetric spaces, Amer. J. Math. 80 (1958), 964-1029. MR MR0105694 (21 \#4430)

[7] Nicolas Bourbaki, Eléments de mathématique: groupes et algèbres de Lie, Masson, Paris, 1982, Chapitre 9. Groupes de Lie réels compacts. [Chapter 9. Compact real Lie groups]. MR 84i:22001

[8] A. K. Bousfield, Homotopy spectral sequences and obstructions, Israel J. Math. 66 (1989), no. 1-3, 54-104. MR MR1017155 (91a:55027)

[9] A. K. Bousfield and D. M. Kan, Homotopy limits, completions and localizations, Lecture Notes in Mathematics, no. 304, Springer-Verlag, Berlin, 1972.

[10] Peter Bouwknegt, Alan L. Carey, Varghese Mathai, Michael K. Murray, and Danny Stevenson, Twisted $K$-theory and K-theory of bundle gerbes, Comm. Math. Phys. 228 (2002), no. 1, 1745. MR 1911247

[11] Kenneth S. Brown, Buildings, Springer-Verlag, New York, 1989. MR 90e:20001

[12] U. Bunke and I. Schröder, Twisted K-theory and TQFT, Mathematisches Institut, Georg-August-Universität Göttingen: Seminars Winter Term 2004/2005, Universitätsdrucke Göttingen, Göttingen, 2005, pp. 33-80. MR MR2206878 (2007c:19008)

[13] Albrecht Dold, Partitions of unity in the theory of fibrations, Ann. of Math. (2) 78 (1963), 223-255. MR 27 \#5264

[14] P. Donovan and M. Karoubi, Graded Brauer groups and K-theory with local coefficients, Inst. Hautes Études Sci. Publ. Math. (1970), no. 38, 5-25. MR 43 \#8075

[15] Christopher L. Douglas, On the twisted K-homology of simple Lie groups, Topology 45 (2006), no. 6, 955-988. MR MR2263220

[16] J. J. Duistermaat and J. A. C. Kolk, Lie groups, Universitext, Springer-Verlag, Berlin, 2000. MR MR1738431 (2001j:22008)

[17] Daniel S. Freed, Higher algebraic structures and quantization, Comm. Math. Phys. 159 (1994), no. 2, 343-398. MR MR1256993 (95c:58034)

[18] Daniel S. Freed, Michael J. Hopkins, and Constantin Teleman, Consistent orientation of moduli spaces.

[19] Loop Groups and Twisted K-Theory II, arXiv:math.AT/0511232

[20] L Loop Groups and Twisted K-theory III, arXiv:math.AT/0312155

[21] — , Twisted equivariant K-theory with complex coefficients, arXiv:math.AT/0206257. accepted for publication by Topology.

[22] Sigurdur Helgason, Differential geometry, Lie groups, and symmetric spaces, Graduate Studies in Mathematics, vol. 34, American Mathematical Society, Providence, RI, 2001, Corrected reprint of the 1978 original. MR 2002b:53081

[23] Michael Joachim, A symmetric ring spectrum representing KO-theory, Topology 40 (2001), no. 2, 299-308. MR MR1808222 (2001k:55011)

[24] Nitu Kitchloo, Dominant K-theory and highest weight representations of Kac-Moody groups, preprint.

[25] L. G. Lewis, J. P. May, and M. Steinberger, Equivariant stable homotopy theory, Lecture Notes in Mathematics, vol. 1213, Springer-Verlag, New York, 1986. 
[26] Ruben Minasian and Gregory Moore, K-theory and Ramond-Ramond charge, J. High Energy Phys. (1997), no. 11, Paper 2, 7 pp. (electronic). MR 2000a:81190

[27] G. D. Mostow, Equivariant embeddings in Euclidean space, Ann. of Math. (2) 65 (1957), 432-446. MR MR0087037 (19,291c)

[28] Richard S. Palais, On the existence of slices for actions of non-compact Lie groups, Ann. of Math. (2) 73 (1961), 295-323. MR MR0126506 (23 \#A3802)

[29] Jonathan Rosenberg, Continuous-trace algebras from the bundle theoretic point of view, J. Austral. Math. Soc. Ser. A 47 (1989), no. 3, 368-381. MR MR1018964 (91d:46090)

[30] G. Segal, Classifying spaces and spectral sequences, Inst. Hautes Études Sci. Publ. Math. 34 (1968), 105-112.

[31] Stephan Stolz and Peter Teichner, What is an elliptic object?, Topology, geometry and quantum field theory, London Math. Soc. Lecture Note Ser., vol. 308, Cambridge Univ. Press, Cambridge, 2004, pp. 247-343. MR MR2079378 (2005m:58048)

[32] Burt Totaro, The resolution property for schemes and stacks, J. Reine Angew. Math. $\mathbf{5 7 7}$ (2004), 1-22. MR MR2108211 (2005j:14002)

[33] Jean-Louis Tu, Ping Xu, and Camille Laurent-Gengoux, Twisted K-theory of differentiable stacks, Ann. Sci. École Norm. Sup. (4) 37 (2004), no. 6, 841-910. MR MR2119241 (2005k:58037)

[34] Erik Verlinde, Fusion rules and modular transformations in $2 D$ conformal field theory, $\mathrm{Nu}-$ clear Phys. B 300 (1988), no. 3, 360-376. MR 89h:81238

[35] Edward Witten, D-branes and K-theory, J. High Energy Phys. (1998), no. 12, Paper 19, 41 pp. (electronic). MR 2000e:81151

Department of Mathematics, University of Texas, Austin, TX

E-mail address: dafr@math.utexas.edu

Department of Mathematics, Massachusetts Institute of Technology, Cambridge, MA 02139-4307

E-mail address: mjh@math.mit.edu

Cambridge, Cambridge

E-mail address: c.teleman 